\title{
General Dual-Switched Dynamic Singlet Fission Channels in Solvents Governed Jointly by Chromophore Structural Dynamics and Solvent Impact: Singlet Pre-Fission
}

\section{Energetics Analyses}

Lijuan Xue, Xinyu Song, Yiwei Feng, Shibo Cheng, Gang Lu, Yuxiang Bu*

School of Chemistry and Chemical Engineering, Shandong University, Jinan, 250100, People's

Republic of China. *e-mail: byx @ sdu.edu.cn

\section{Supporting Information}

\section{Contents}

1. Excited state energies and corresponding molecular orbital energies of static di-BODIPY in the gas phase and solvents and comparison using different functionals

2. Dynamics simulation data of di-BODIPY in the gas phase

3. Electron transitions of excited states and their corresponding frontier molecular orbitals of diBODIPY for the AIMD instantaneous configurations and four possible singlet fission channels in $\mathrm{CHCl}_{3}$

4. Electron transitions of excited states and their corresponding frontier molecular orbitals of diBODIPY for the AIMD instantaneous configurations and two possible singlet fission channels in cyclohexane

5. Transition configurations of the smallest vertical excitation energy of the triplet state

6. Orbital energy correlation and time evolution dynamics and relationship between the CT state energies and ${ }^{1} \mathrm{LUMO}$ energies

7. Further verification of the proposed dual-switched singlet fission channel for Di-BODIPY in frozen DCM/toluene at $80 \mathrm{~K}$

8. Further verification of the proposed dual-switched singlet fission channel using other system (a covalent pentacene dimer in benzonitrile)

9. Additional AIMD simulation for di-BODIPY in $\mathrm{CHCl}_{3}$ (the other trajectory)

10. Time evolution of the dihedral angles and bond lengths

11. Comparison of the $\mathrm{CT}$ state energies and their frontier molecular orbitals of di-BODIPY in $\mathrm{CHCl}_{3}$ and cyclohexane solvent using different functionals

12. Simple introduction about SF mechanisms and the CT-state role 


\section{Excited State Energies and Corresponding Molecular Orbital Energies of Static di-BODIPY in the Gas Phase and Solvents and Comparison Using Different Functionals}

The calculated excited state energies and frontier molecular orbital energies at the optimized ground state structure of di-BODIPY in the gas phase and solvents at the M062X/cc-pVDZ level are given in Table S1 (A). To verify the M062X functional is reliable for this system, excitation energies were calculated with other functionals (B3LYP, PBE0 and CAM-B3LYP) and a ccpVDZ basis set, as shown in Table S1 (B). The values of singlet and triplet excitation energies are slightly different in different methods. Furthermore, $\Delta \mathrm{E}_{S F}$ is greater than 0 at B3LYP and PBE0 method, which is consistent with the result of M062X functional. These indicate that the M062X/cc-pVDZ level is reliable in studying the excitation energetics for the present system.

Table S1. (A) Vertical Excited State Energies and Frontier Molecular Orbital Energies of the Optimized Ground State Structure of di-BODIPY in the Gas Phase and Solvents. The Calculations are at the M062X/cc-pVDZ Level

\begin{tabular}{|c|c|c|c|c|c|c|c|c|c|}
\hline & $S_{1}(e V)$ & ${ }^{2} T_{1}(e V)$ & ${ }^{1} T_{1}(e V)$ & CT(eV) & $\Delta \mathbf{E}_{\mathrm{SF}}(\mathrm{eV})$ & $\Delta \mathrm{E}_{\mathrm{CT}-\mathrm{S1}}(\mathrm{eV})$ & $\begin{array}{c}{ }^{2} \mathrm{HOMO} \\
\text { energy }(\mathrm{eV})\end{array}$ & $\begin{array}{c}{ }^{2} \mathrm{LUMO} \\
\text { energy(eV) }\end{array}$ & $\begin{array}{c}{ }^{2} \mathbf{H}^{2} \mathbf{L} \\
\text { GAP(eV) }\end{array}$ \\
\hline Gas phase & 2.949 & 1.484 & 1.511 & 3.154 & 0.046 & 0.205 & -6.55 & -1.81 & 4.74 \\
\hline $\mathrm{CHCl}_{3}$ & 2.836 & 1.505 & 1.547 & 3.259 & 0.216 & 0.423 & -6.71 & -1.93 & 4.78 \\
\hline cyclohexane & 2.837 & 1.506 & 1.565 & 3.322 & 0.234 & 0.485 & -6.70 & -1.93 & 4.77 \\
\hline
\end{tabular}

Table S1. (B) Vertical Excited State Energies of the Optimized Ground State Structures of the Gaseous Static di-BODIPY with the B3LYP, PBE0, CAM-B3LYP Functionals, EOM-CCSD Method and a cc-pVDZ Basis Set

\begin{tabular}{|c|c|c|c|c|c|c|}
\hline & $\mathbf{S}_{\mathbf{1}}(\mathbf{e V})$ & ${ }^{2} \mathbf{T}_{\mathbf{1}}(\mathbf{e V})$ & ${ }^{{ }^{1} \mathbf{T}_{\mathbf{1}}(\mathbf{e V})}$ & $\mathbf{C T}(\mathbf{e V})$ & $\Delta \mathbf{E}_{\mathbf{S F}}(\mathbf{e V})$ & $\Delta \mathbf{E}_{\mathbf{C T}-\mathrm{S1}}(\mathbf{e V})$ \\
\hline M062X & 2.949 & 1.484 & 1.511 & 3.154 & 0.046 & 0.205 \\
\hline B3LYP & 2.954 & 1.481 & 1.601 & 3.369 & 0.128 & 0.415 \\
\hline PBE0 & 2.816 & 1.412 & 1.507 & 3.017 & 0.103 & 0.201 \\
\hline CAM-B3LYP & 2.968 & 1.504 & 1.629 & 3.331 & 0.165 & 0.363 \\
\hline
\end{tabular}

Note: $\Delta \mathrm{E}_{\mathrm{SF}}=\mathrm{E}\left({ }^{2} \mathrm{~T}_{1}\right)+\mathrm{E}\left({ }^{1} \mathrm{~T}_{1}\right)-\mathrm{E}\left(\mathrm{S}_{1}\right) ; \Delta \mathrm{E}_{\mathrm{CT}-\mathrm{S} 1}=\mathrm{E}(\mathrm{CT})-\mathrm{E}\left(\mathrm{S}_{1}\right)$ 
Table S2. Frontier Molecular Orbitals of di-BODIPY in the Gas Phase

\begin{tabular}{|c|c|c|c|}
\hline Gas phase & BODIPY(1) & & BODIPY(2) \\
\hline $\begin{array}{c}{ }^{1} \mathrm{LUMO}+1 \\
(136)\end{array}$ & & $\begin{array}{c}{ }^{2} \mathrm{LUMO}+1 \\
\quad(138)\end{array}$ & \\
\hline $\begin{array}{c}{ }^{1} \text { LUMO } \\
\text { (134) }\end{array}$ & & $\begin{array}{c}{ }^{2} \text { LUMO } \\
(135)\end{array}$ & \\
\hline $\begin{array}{c}{ }^{1} \mathrm{HOMO} \\
(132)\end{array}$ & & $\begin{array}{c}{ }^{2} \mathrm{HOMO} \\
(133)\end{array}$ & \\
\hline $\begin{array}{c}{ }^{1} \mathrm{HOMO}-1 \\
(129)\end{array}$ & & $\begin{array}{c}{ }^{2} \mathrm{HOMO}-1 \\
(131)\end{array}$ & \\
\hline
\end{tabular}

Note: ${ }^{1} \mathrm{HOMO}$ and ${ }^{1} \mathrm{LUMO}$ represent the frontier molecular orbitals of BODIPY(1), while ${ }^{2} \mathrm{HOMO}$ and ${ }^{2}$ LUMO represent those of BODIPY(2).

Table S3. Electron Transitions of Excited States and Their Corresponding Frontier Molecular Orbitals of the Optimized Ground State Structure of di-BODIPY in the Gas Phase

\begin{tabular}{|c|c|c|c|c|c|}
\hline Solvent & $\begin{array}{c}\text { Excited } \\
\text { states }\end{array}$ & Configuration & $\begin{array}{c}\text { Energy } \\
(\mathrm{eV})\end{array}$ & $\begin{array}{c}\text { Orbital } \\
\text { (occupied) }\end{array}$ & $\begin{array}{c}\text { Orbital } \\
\text { (unoccupied) }\end{array}$ \\
\hline \multirow{5}{*}{$\begin{array}{l}\text { Gas } \\
\text { phase }\end{array}$} & ${ }^{1} S_{1}$ & $\begin{array}{c}132->134 \quad 0.57551 \\
{ }^{1} \mathrm{HOMO} \rightarrow{ }^{1} \text { LUMO } \\
(0.57551)\end{array}$ & 2.949 & & \\
\hline & ${ }^{2} \mathrm{~S}_{1}$ & $\begin{array}{c}133->135 \quad 0.54836 \\
{ }^{2} \mathrm{HOMO} \rightarrow{ }^{2} \mathrm{LUMO} \\
(0.54836)\end{array}$ & 2.968 & & \\
\hline & $\mathrm{CT}$ & $\begin{array}{c}133->134 \quad 0.67310 \\
{ }^{2} \mathrm{HOMO} \rightarrow{ }^{1} \text { LUMO } \\
(0.67310)\end{array}$ & 3.154 & & \\
\hline & ${ }^{2} \mathrm{~T}_{1}$ & $\begin{array}{c}133->135 \quad 0.69572 \\
{ }^{2} \mathrm{HOMO} \rightarrow{ }^{2} \mathrm{LUMO} \\
(0.69572)\end{array}$ & 1.484 & & \\
\hline & ${ }^{1} \mathrm{~T}_{1}$ & $\begin{array}{c}132->134 \quad 0.69896 \\
{ }^{1} \mathrm{HOMO} \rightarrow{ }^{1} \mathrm{LUMO} \\
(0.69896)\end{array}$ & 1.511 & & \\
\hline
\end{tabular}


Table S4. Frontier Molecular Orbitals of di-BODIPY in $\mathrm{CHCl}_{3}$

\begin{tabular}{|c|c|c|c|}
\hline $\mathrm{CHCl}_{3}$ & BODIPY(1) & & BODIPY(2) \\
\hline $\begin{array}{c}{ }^{1} \text { LUMO+1 } \\
\quad(136)\end{array}$ & & $\begin{array}{c}{ }^{2} \mathrm{LUMO}+1 \\
\quad(137)\end{array}$ & \\
\hline $\begin{array}{l}{ }^{1} \text { LUMO } \\
(134)\end{array}$ & & $\begin{array}{c}{ }^{2} \text { LUMO } \\
(135)\end{array}$ & \\
\hline $\begin{array}{c}{ }^{1} \mathrm{HOMO} \\
(132)\end{array}$ & & $\begin{array}{c}{ }^{2} \mathrm{HOMO} \\
(133)\end{array}$ & \\
\hline $\begin{array}{c}{ }^{1} \mathrm{HOMO}-1 \\
(130)\end{array}$ & & $\begin{array}{c}{ }^{2} \mathrm{HOMO}-1 \\
\text { (131) }\end{array}$ & \\
\hline
\end{tabular}

Table S5. Electron Transitions of Excited States and Their Corresponding Frontier Molecular Orbitals in the Optimized Ground State Structure of di-BODIPY in $\mathrm{CHCl}_{3}$

\begin{tabular}{|c|c|c|c|c|c|}
\hline Solvent & $\begin{array}{c}\text { Excited } \\
\text { states }\end{array}$ & Configuration & $\begin{array}{c}\text { Energy } \\
(\mathrm{eV})\end{array}$ & $\begin{array}{c}\text { Orbital } \\
\text { (occupied) }\end{array}$ & $\begin{array}{c}\text { Orbital } \\
\text { (unoccupied) }\end{array}$ \\
\hline \multirow{5}{*}{$\mathrm{CHCl}_{3}$} & ${ }^{2} \mathrm{~S}_{1}$ & $\begin{array}{c}133->135 \quad 0.53825 \\
{ }^{2} \mathrm{HOMO} \rightarrow{ }^{2} \text { LUMO } \\
(0.53825)\end{array}$ & 2.836 & & \\
\hline & ${ }^{1} \mathrm{~S}_{1}$ & $\begin{array}{c}132->134 \quad 0.64481 \\
{ }^{1} \mathrm{HOMO} \rightarrow{ }^{1} \text { LUMO } \\
(0.64481)\end{array}$ & 2.862 & & \\
\hline & CT & $\begin{array}{c}133->134 \quad 0.56074 \\
{ }^{2} \mathrm{HOMO} \rightarrow{ }^{1} \text { LUMO } \\
(0.56074)\end{array}$ & 3.371 & & \\
\hline & ${ }^{2} \mathrm{~T}_{1}$ & $\begin{array}{c}133->135 \quad 0.59663 \\
{ }^{2} \mathrm{HOMO} \rightarrow{ }^{2} \mathrm{LUMO} \\
(0.59663)\end{array}$ & 1.505 & & \\
\hline & ${ }^{1} \mathrm{~T}_{1}$ & $\begin{array}{c}132->134 \quad 0.61660 \\
{ }^{1} \mathrm{HOMO} \rightarrow{ }^{1} \mathrm{LUMMO} \\
(0.61660) \\
\end{array}$ & 1.547 & & \\
\hline
\end{tabular}


Table S6. Frontier Molecular Orbitals of di-BODIPY in cyclohexane

\begin{tabular}{cccc}
\hline cyclohexane BODIPY(1) & & BODIPY(2) \\
${ }^{1}$ LUMO+1 \\
$(136)$
\end{tabular}

Table S7. Electron Transitions of Excited States and Their Corresponding Frontier Molecular Orbitals of the Optimized Ground State Structure of di-BODIPY in cyclohexane

\begin{tabular}{|c|c|c|c|c|c|}
\hline Solvent & Excited states & Configuration & $\begin{array}{c}\text { Energy } \\
(\mathrm{eV})\end{array}$ & $\begin{array}{c}\text { Orbital } \\
\text { (occupied) }\end{array}$ & $\begin{array}{c}\text { Orbital } \\
\text { (unoccupied) }\end{array}$ \\
\hline \multirow{5}{*}{ cyclohexane } & ${ }^{2} \mathrm{~S}_{1}$ & $\begin{array}{c}133->135 \quad 0.53904 \\
{ }^{2} \mathrm{HOMO} \rightarrow{ }^{2} \mathrm{LUMO} \\
(0.53904)\end{array}$ & 2.837 & & \\
\hline & ${ }^{1} \mathrm{~S}_{1}$ & $\begin{array}{c}132->134 \quad 0.64177 \\
{ }^{1} \mathrm{HOMO} \rightarrow{ }^{1} \mathrm{LUMO} \\
(0.64177)\end{array}$ & 2.863 & & \\
\hline & CT & $\begin{array}{c}133->134 \quad 0.56528 \\
{ }^{2} \mathrm{HOMO} \rightarrow{ }^{1} \mathrm{LUMO} \\
(0.56528)\end{array}$ & 3.390 & & \\
\hline & ${ }^{2} \mathrm{~T}_{1}$ & $\begin{array}{c}133->135 \quad 0.60098 \\
{ }^{2} \mathrm{HOMO} \rightarrow{ }^{2} \mathrm{LUMO} \\
(0.60098)\end{array}$ & 1.506 & & \\
\hline & ${ }^{1} \mathrm{~T}_{1}$ & $\begin{array}{c}132->134 \quad 0.61926 \\
{ }^{1} \mathrm{HOMO} \rightarrow{ }^{1} \text { LUMO } \\
(0.61926)\end{array}$ & 1.565 & & \\
\hline
\end{tabular}


Table S8. The HOMO and LUMO Distributions of di-BODIPY and Its Complexes with One and Two $\mathrm{CHCl}_{3}$ Molecules

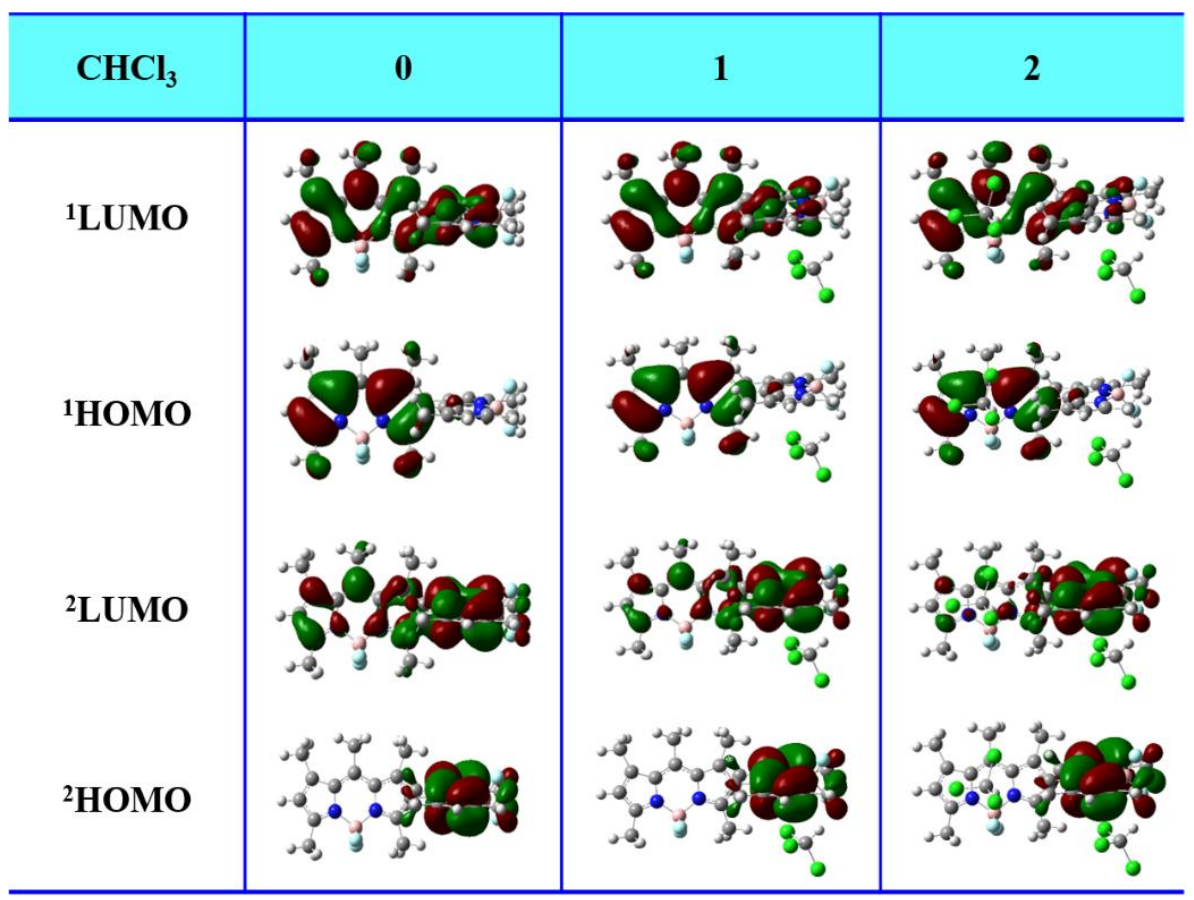

Table S9. The HOMO and LUMO Distributions of di-BODIPY and Its Complexes with One and Two Cyclohexane Molecules

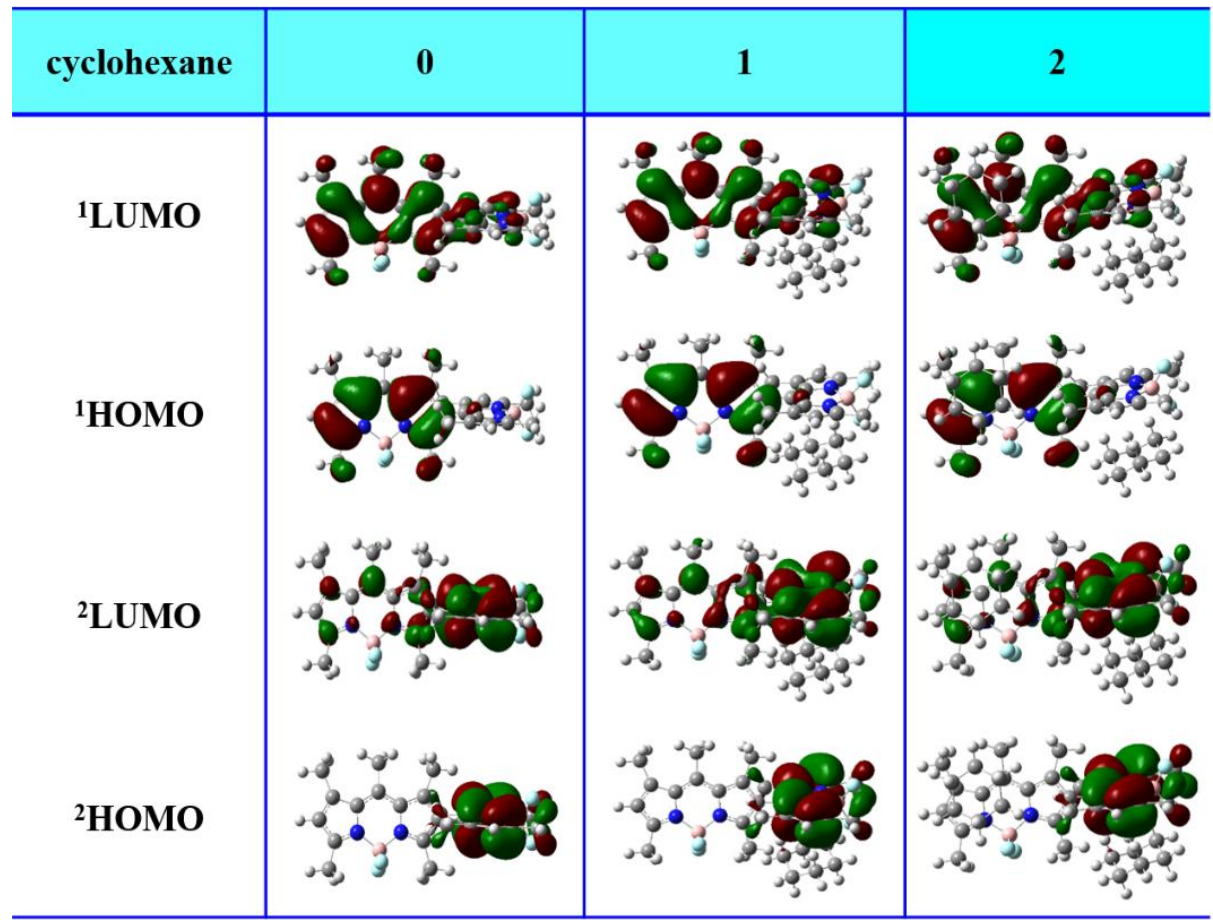




\section{Dynamics Simulation Data of di-BODIPY in the Gas Phase}

Relevant results in the gas phase are shown in Figure S1. In Figure S1(a), the probability of meeting SF energy criterion in gaseous phase is only $3 \%$, indicating that di-BODIPY hardly undergo SF in the gas phase even though at its deformed geometries and structure perturbation of chromophore doesn't provide sufficient condition for SF alone. There is no direct relationship between $\mathrm{E}\left(\mathrm{S}_{1}\right)$ and $\Delta \mathrm{E}_{\mathrm{SF}}($ Figure $\mathbf{S 1}(\mathbf{b}))$. Unlike $\mathrm{E}\left(\mathrm{S}_{1}\right), \Delta \mathrm{E}_{\mathrm{SF}}$ presents a good linear correlation with $\mathrm{E}\left({ }^{1} \mathrm{~T}_{1}\right), \mathrm{E}\left({ }^{2} \mathrm{~T}_{1}\right)$ and the sum of $\mathrm{E}\left({ }^{1} \mathrm{~T}_{1}\right)$ and $\mathrm{E}\left({ }^{2} \mathrm{~T}_{1}\right)$ (Figure $\left.\mathrm{S1}(\mathbf{c})\right)$. Similar to $\mathrm{E}\left(\mathrm{S}_{1}\right)$ and $\Delta \mathrm{E}_{\mathrm{SF}}$ (Figure S1(a)), there is no linear relationship between $\Delta \mathrm{E}_{\mathrm{CT}-\mathrm{S} 1}$ and $\mathrm{E}\left(\mathrm{S}_{1}\right)$ (Figure S1(d)). However, there is a linear correlation between $\Delta \mathrm{E}_{\mathrm{CT}-\mathrm{S} 1}$ and $\mathrm{E}(\mathrm{CT})$ in the gaseous phase (Figure S1(d)).

The relationship between $\mathrm{E}\left({ }^{1} \mathrm{~T}_{1}\right) / \mathrm{E}\left({ }^{2} \mathrm{~T}_{1}\right)$ and ${ }^{1} \mathrm{HOMO}-{ }^{1} \mathrm{LUMO} /{ }^{2} \mathrm{HOMO}-{ }^{2} \mathrm{LUMO}$ energy gap is analyzed in the gaseous phase, as shown in Figure S2(a). There is a linear correlation between $\mathrm{E}\left({ }^{1} \mathrm{~T}_{1}\right)$ and the ${ }^{1} \mathrm{HOMO}-{ }^{1} \mathrm{LUMO}$ energy gaps in the gaseous phase. The relationship between $\mathrm{E}\left({ }^{2} \mathrm{~T}_{1}\right)$ and the ${ }^{2} \mathrm{HOMO}-{ }^{2} \mathrm{LUMO}$ is also a good linear correlation. This indicates that $\mathrm{E}\left(\mathrm{T}_{1}\right)$ is determined by the HOMO-LUMO energy gap. The relationships between the ${ }^{1} \mathrm{LUMO}$ energy and the $\mathrm{CT}$ state energy or $\Delta \mathrm{E}_{\mathrm{CT}-\mathrm{S} 1}$ is also examined in the gaseous phase, as illustrated in Figure S2(b). Figure S2(b) displays the CT state energies or $\Delta \mathrm{E}_{\mathrm{CT} \text {-S1 }}$ and ${ }^{1} \mathrm{LUMO}$ energies with a quasilinear correlation between them and the $\mathrm{CT}$ state energy or $\Delta \mathrm{E}_{\mathrm{CT}-\mathrm{S} 1}$ increases gradually with arising of the ${ }^{1}$ LUMO energy. These results are similar to those in solvents.

We also explore the origin of the effect of the inner-sphere interaction between chromophore and solvent molecules on the energies. The direct inner-sphere interaction with solvent molecules is explicitly taken into account. Calculations indicate that the combined effect of direct inner-sphere solvent interaction and outer-sphere solvent polarity reduces both the excitation energies of $S_{1}$ and $T_{1}$ simultaneously. As shown in Figure S3(c), the $S_{1}$ excitation energy of di-BODIPY in $\mathrm{CHCl}_{3}$ in molecular cluster models is reduced from $2.836 \mathrm{eV}$ (in PCM model without explicit solvent molecules) to $2.817 \mathrm{eV}$, while the ${ }^{2} \mathrm{~T}_{1}$ excitation energy decreases from $1.505 \mathrm{eV}$ to $1.436 \mathrm{eV}$ and ${ }^{1} \mathrm{~T}_{1}$ excitation decreases from $1.547 \mathrm{eV}$ (PCM) to $1.489 \mathrm{eV}$. The excitation energies of di-BODIPY in c-hex has a similar tendency, but the magnitudes are slightly different. Clearly, the ${ }^{1} \mathrm{~T}_{1} /{ }^{2} \mathrm{~T}_{1}$ excitation energies have relatively larger decreases compared 
with the $\mathrm{S}_{1}$ excitation energy $(0.058 / 0.069 \mathrm{eV}$ versus $0.019 \mathrm{eV})$, and thus such variations are favorable to the driving force of SF. Clearly, it can be expected that chromophore interacting directly with more solvent molecules could get more favorable driving force for SF, as evidenced in the results from the sampling configurations.

We further make an analysis on the reliability of the TD-M062X/CC-pVDZ method as the followings. In fact, we made relevant calculations at the high level wave-function-correlated EOM-CCSD method, but we only make partial analysis in the previous responses.

The results of the high-level wave-function-correlated EOM-CCSD method are consistent with M062X method. For the CT state energies of gaseous static di-BODIPY, the TD-M062X result $(3.154 \mathrm{eV})$ is close to the TD-EOM-CCSD value $(3.290 \mathrm{eV})$, and for two switching quantities $\left(\Delta \mathrm{E}_{\mathrm{SF}}, \Delta \mathrm{E}_{\mathrm{CT}-\mathrm{S} 1}\right)$, the TD-M062X values $(0.046$ and $0.205 \mathrm{eV})$ are quite close to the TDEOM-CCSD ones (0.092 and $0.224 \mathrm{eV})$. Furthermore, we calculated the changes of vertical excitation energies of all sampling configurations under intermolecular interaction between diBODIPY and $\mathrm{CHCl}_{3} / \mathrm{c}$-hex solvent molecules using the TD-M062X and TD-EOM-CCSD method, as shown in Figure S3 (c,d). At the M062X/cc-pVDZ level, the excitation energy $\mathrm{S}_{1}$, ${ }^{1} \mathrm{~T}_{1},{ }^{2} \mathrm{~T}_{1}$ and CT of di-BODIPY in $\mathrm{CHCl}_{3}$ and in c-hex decrease with the increase of solvent molecule interaction. The results of the EOM-CCSD/cc-pVDZ level have a similar tendency, but the magnitudes are slightly different (Figure S3 (c)). Most of all, $\Delta \mathrm{E}_{\mathrm{SF}}$ and $\Delta \mathrm{E}_{\mathrm{CT}-\mathrm{S} 1}$ calculated using two methods are very close to each other, as shown in Figure S3 (d).

Besides, we also compared the experimental and theoretical values of di-BODIPY in $\mathrm{CHCl}_{3}$, the vertical excitation energies of singlet and triplet states actually agree well with other calculated and experimental values. We calculated the adiabatic excitation energies of BODIPYs 10 in ref Buck et al., Chem 2019 at the M062X/CC-pVDZ level, and the calculated values are quite close to the experimental values. Various excitation energies of the gaseous static di-BODIPY were calculated using other functionals B3LYP, PBE0 and CAM-B3LYP with the cc-pVDZ basis set at the TDDFT framework and we also used the B3LYP and CAM-B3LYP methods to calculate the $\mathrm{CT}$ excitation energies and $\Delta \mathrm{E}_{\mathrm{CT}-\mathrm{S} 1}$ of some AIMD sampling configurations of di-BODIPY in $\mathrm{CHCl}_{3}$ and c-hex solvents, and all results are in good agreement for several methods. 
(a)

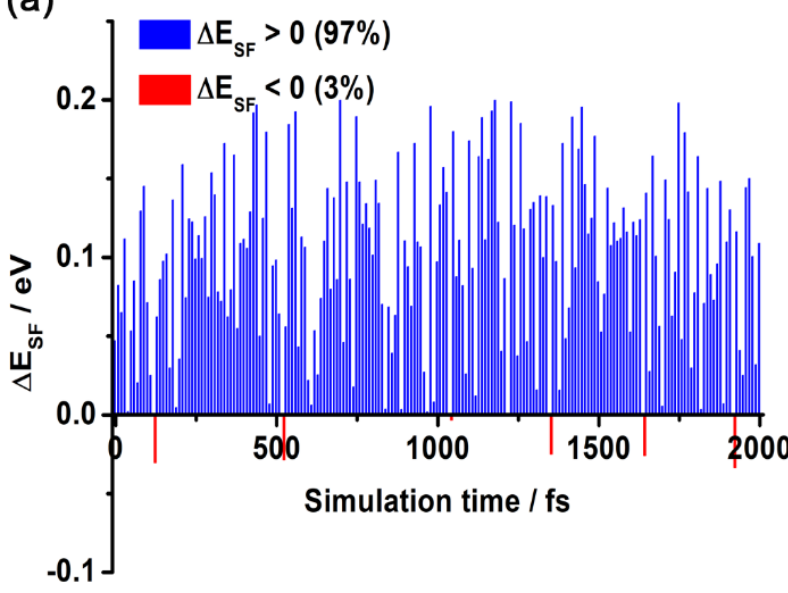

(c)

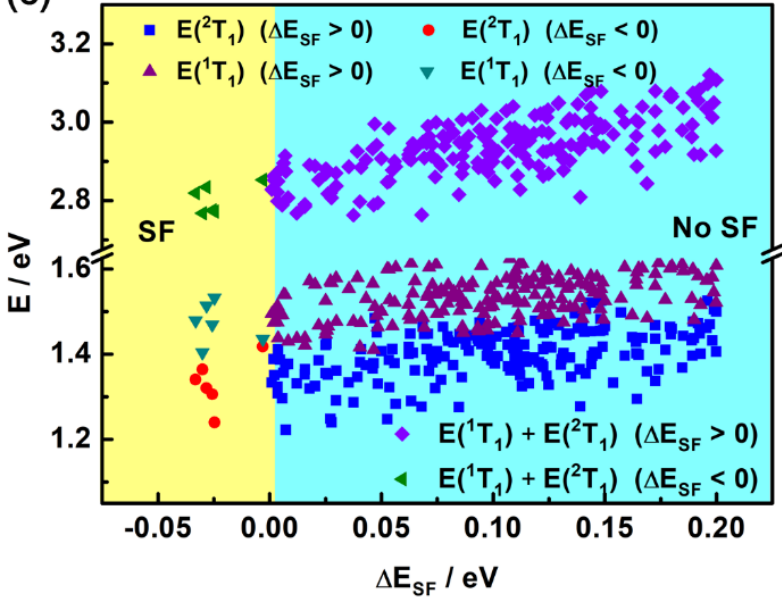

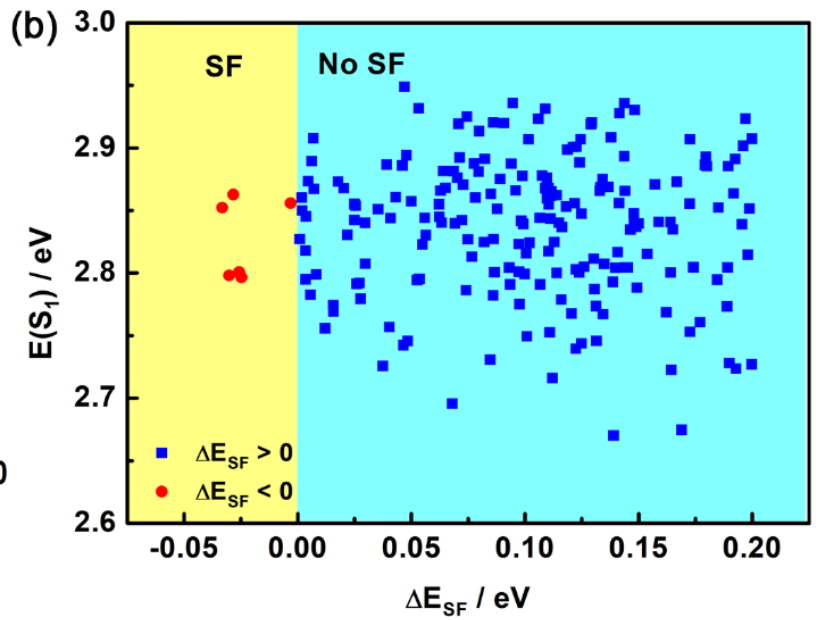

(d)

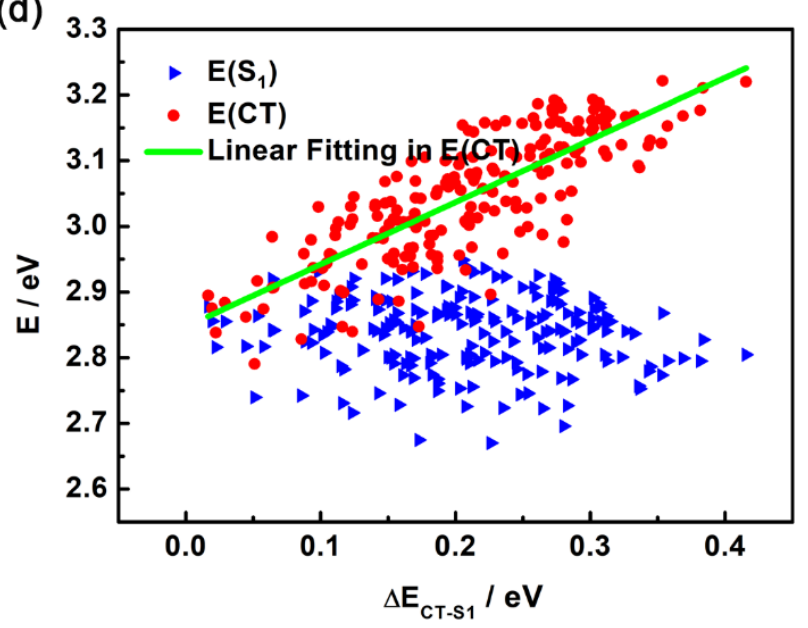

Figure S1. (a) Relevant results in the gas phase. The SF driving force $\left(\Delta \mathrm{E}_{\mathrm{SF}}\right)$ of di-BODIPY in the gas phase for all 200 instantaneous configurations extracted every 10 fs from a 2 ps AIMD simulation trajectory. $\Delta \mathrm{E}_{\mathrm{SF}} \leq 0$ means that di-BODIPY meets the $\mathrm{SF}$ thermodynamic criterion in the gas phase (red bars), while $\Delta \mathrm{E}_{\mathrm{SF}}>0$ means that di-BODIPY cannot undergo SF in the gas phase (blue bars). The probability of meeting SF energy requirement in the gas phase is only $3 \%$. (b) The relationship between the $\mathrm{SF}$ driving force $\left(\Delta \mathrm{E}_{\mathrm{SF}}\right)$ and smallest singlet state vertical excitation energies $\left(E\left(S_{1}\right)\right)$. Red dots $\left(\Delta E_{S F} \leq 0\right)$ denote that di-BODIPY meets the SF thermodynamic criterion in the gas phase and blue dots $\left(\Delta \mathrm{E}_{\mathrm{SF}}>0\right)$ denote that di-BODIPY does not in the gas phase. (c) The relationship between the SF driving force $\left(\Delta \mathrm{E}_{S F}\right)$ and $\mathrm{E}\left({ }^{2} \mathrm{~T}_{1}\right)$ or $\mathrm{E}\left({ }^{1} \mathrm{~T}_{1}\right)$ or the sum of $\mathrm{E}\left({ }^{1} \mathrm{~T}_{1}\right)$ and $\mathrm{E}\left({ }^{2} \mathrm{~T}_{1}\right)$, respectively. The red, dark cyan and olive $\left(\Delta \mathrm{E}_{\mathrm{SF}} \leq 0\right)$ 
dots denote the di-BODIPY snapshot configurations meet the SF thermodynamic criterion in the gas phase, while the blue, purple and violet $\left(\Delta \mathrm{E}_{\mathrm{SF}}>0\right)$ dots represent that the di-BODIPY snapshot configurations don't meet the SF energy requirement in the gas phase, respectively.

The relationship between the relative energies ( $\left.\Delta \mathrm{E}_{\mathrm{CT}-\mathrm{S} 1}\right)$ of the $\mathrm{CT}$ state to the $\mathrm{S}_{1}$ state and smallest vertical excitation energy intervals of the singlet state $\left(E\left(S_{1}\right)\right)$ (red dots) of di-BODIPY in the gas phase for 200 snapshot configurations. The relationship between $\Delta \mathrm{E}_{\mathrm{CT}-\mathrm{S} 1}$ and the $\mathrm{CT}$ state energy $(\mathrm{E}(\mathrm{CT}))$ (blue dots) are also displayed.
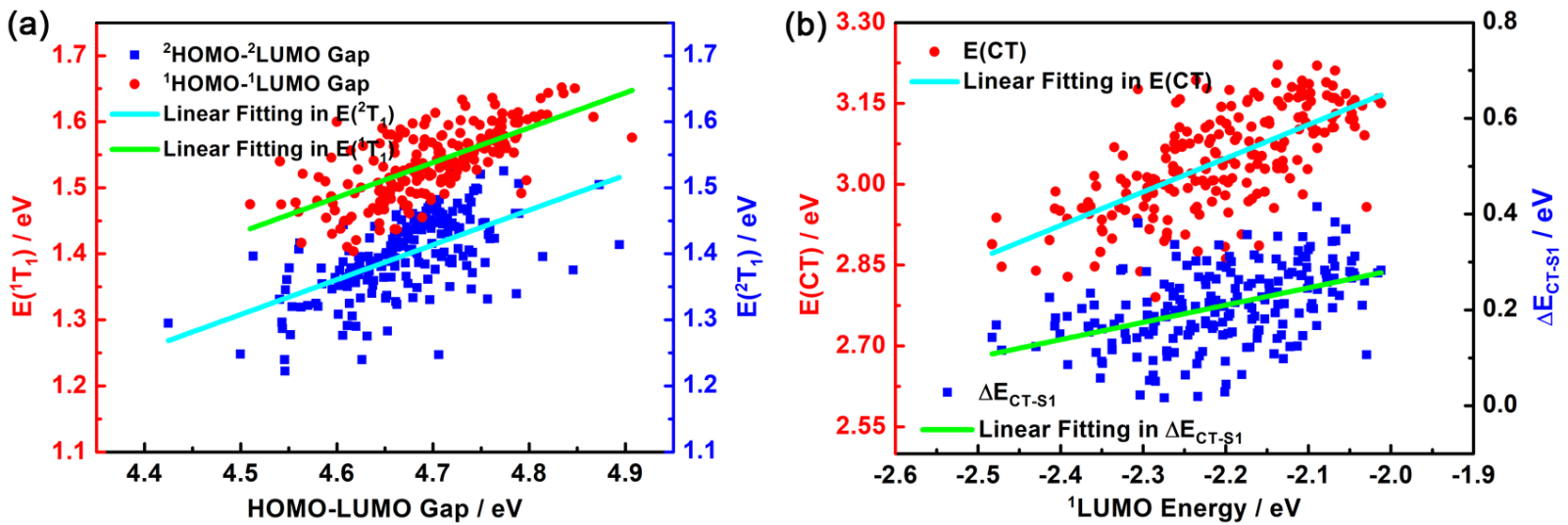

Figure S2. (a) The relationship between the ${ }^{1} \mathrm{HOMO}-{ }^{1} \mathrm{LUMO}$ energy gap and $\mathrm{E}\left({ }^{1} \mathrm{~T}_{1}\right)$ (red dots) of di-BODIPY in the gas phase for 200 snapshot configurations. The relationship between the ${ }^{2} \mathrm{HOMO}-{ }^{2} \mathrm{LUMO}$ energy gap and $\mathrm{E}\left({ }^{2} \mathrm{~T}_{1}\right)$ (blue dots) are also displayed. (b) The relationship between the ${ }^{1}$ LUMO energies and CT state energies $(\mathrm{E}(\mathrm{CT}))$ (red dots) or $\Delta \mathrm{E}_{\mathrm{CT}-\mathrm{S} 1}$ (blue dots) of di-BODIPY in the gas phase for 200 snapshot configurations, respectively. 

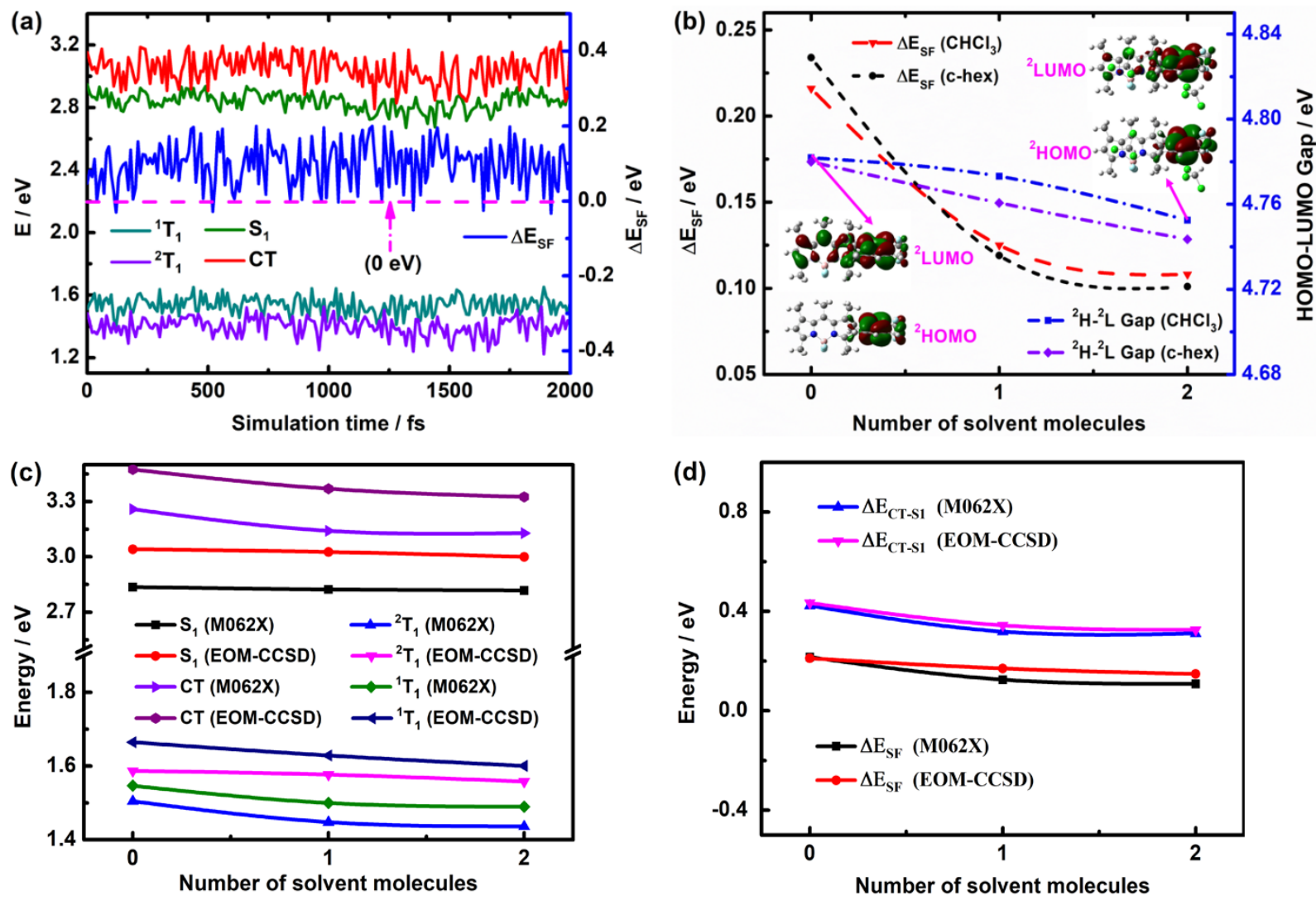

Figure S3. Solvent effect analyses. (a) Time evolutions of the smallest vertical excitation energies to the singlet excited state $\left(\mathrm{S}_{0} \rightarrow \mathrm{S}_{1}, \mathrm{E}\left(\mathrm{S}_{1}\right) /\right.$ red curve $)$ and triplet excited state $\left(\mathrm{S}_{0} \rightarrow{ }^{2} \mathrm{~T}_{1}\right.$, $\mathrm{E}\left({ }^{2} \mathrm{~T}_{1}\right) /$ olive curve $)$ and the SF driving force ( $\Delta \mathrm{E}_{\mathrm{SF}}$, blue curve) of di-BODIPY in the gas phase. (b) Intermolecular interaction (between di-BODIPY and $\mathrm{CHCl}_{3} / \mathrm{c}$-hex solvent molecules) dependence of the SF driving force $\left(\Delta \mathrm{E}_{\mathrm{SF}}\right.$, red dash and black short dash lines) and ${ }^{2} \mathrm{HOMO}-{ }^{2}$ LUMO energy gap (blue dash-dot and purple dash-dot-dot lines). Inserted pictures represent the ${ }^{2} \mathrm{HOMO}$ and ${ }^{2} \mathrm{LUMO}$ distributions of di-BODIPY interacted by two $\mathrm{CHCl}_{3}$ molecules, respectively. Here, $\Delta \mathrm{E}_{\mathrm{SF}}>0$ denotes that di-BODIPY doesn't meet $\mathrm{SF}$ energy requirement in both solvents. (c) Intermolecular interaction (between di-BODIPY and $\mathrm{CHCl}_{3}$ solvent molecules) dependence of the vertical excitation energies of the singlet excited state $\left(\mathrm{S}_{0} \rightarrow \mathrm{S}_{1}, \mathrm{E}\left(\mathrm{S}_{1}\right)\right)$ and triplet excited state $\left(\mathrm{S}_{0} \rightarrow{ }^{2} \mathrm{~T}_{1} /{ }^{1} \mathrm{~T}_{1}, \mathrm{E}\left({ }^{2} \mathrm{~T}_{1}\right) / \mathrm{E}\left({ }^{1} \mathrm{~T}_{1}\right)\right)$ and $\mathrm{CT}$ state energy with the M062X and EOM-CCSD methods and the cc-pVDZ basis set, respectively. (d) Intermolecular interaction (between di-BODIPY and $\mathrm{CHCl}_{3}$ solvent molecules) dependence of the SF driving

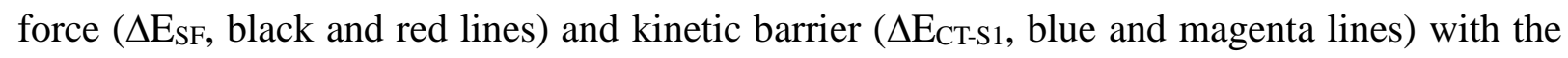
M062X and EOM-CCSD methods and the cc-pVDZ basis set, respectively. 


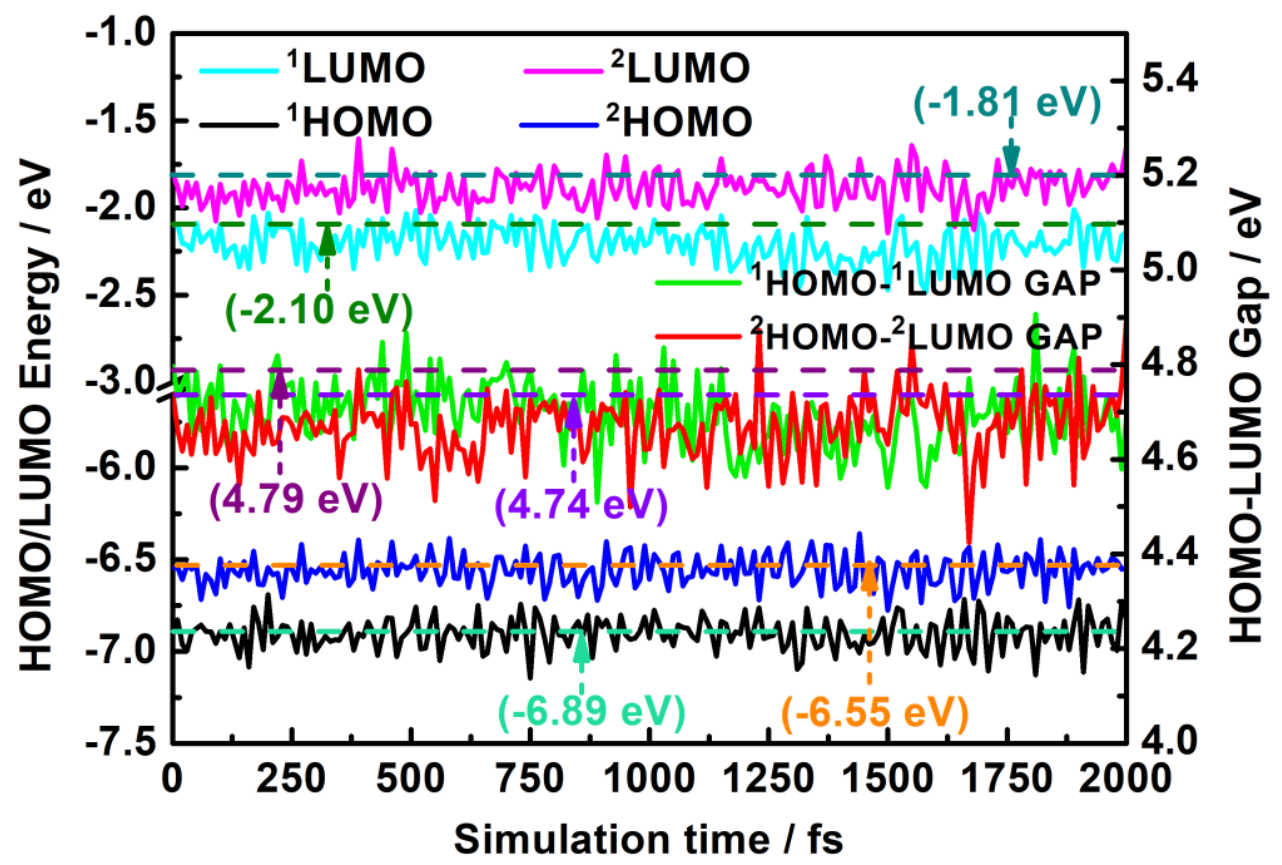

Figure S4. The energy levels of ${ }^{1} \mathrm{HOMO},{ }^{1} \mathrm{LUMO},{ }^{2} \mathrm{HOMO}$ and ${ }^{2} \mathrm{LUMO}$, the ${ }^{1} \mathrm{HOMO}-{ }^{1} \mathrm{LUMO}$ energy gaps and ${ }^{2} \mathrm{HOMO}-{ }^{2}$ LUMO energy gaps of di-BODIPY in the gas phase for all 200 instantaneous configurations extracted every 10 fs from their corresponding 2 ps AIMD simulation trajectories, respectively. The ${ }^{1} \mathrm{HOMO}(-6.89 \mathrm{eV})$ or ${ }^{1} \mathrm{LUMO}(-2.10 \mathrm{eV})$ or ${ }^{2} \mathrm{HOMO}(-$ $6.55 \mathrm{eV})$ or ${ }^{2} \mathrm{LUMO}(-1.81 \mathrm{eV})$ energies and the ${ }^{1} \mathrm{HOMO}-{ }^{1} \mathrm{LUMO}$ energy gap (4.79 eV) or ${ }^{2} \mathrm{HOMO}-{ }^{2} \mathrm{LUMO}$ energy gap $(4.74 \mathrm{eV})$ of the static di-BODIPY molecule are also shown for comparison (light green, olive, orange, dark cyan, purple and violet dash-dot lines). 


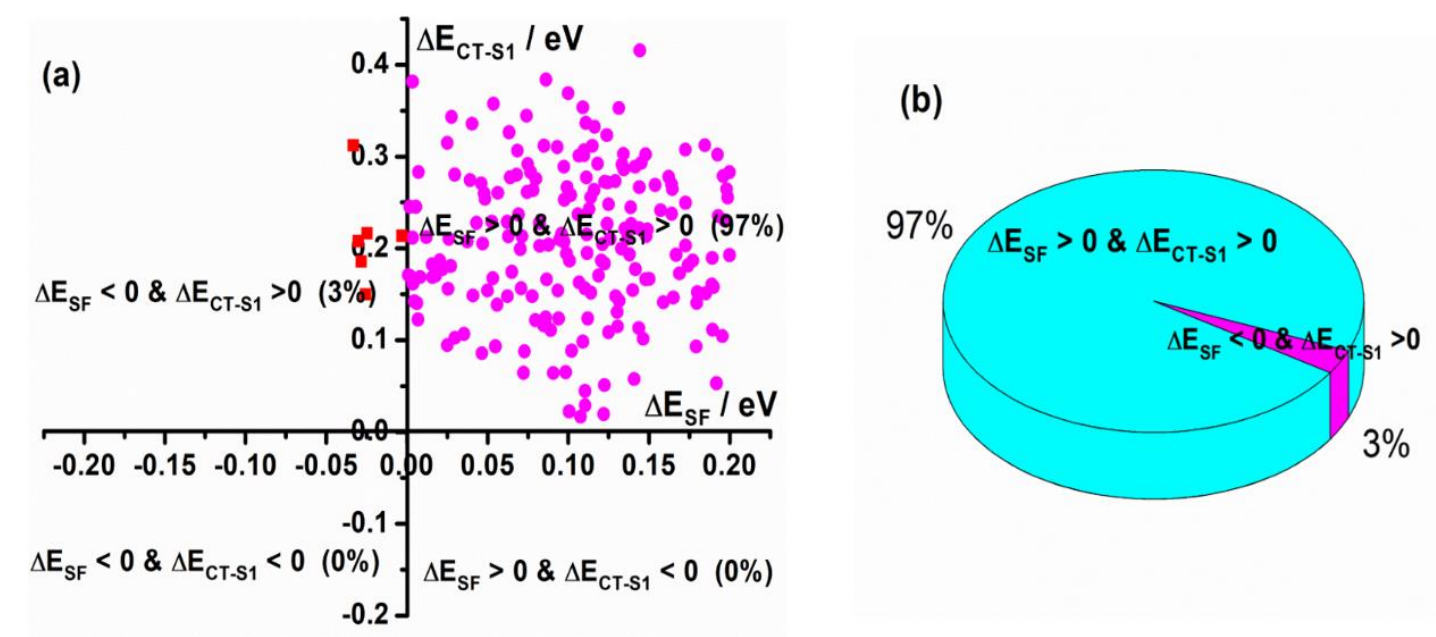

Figure S5. (a) The relationship between thermodynamics $\left(\Delta \mathrm{E}_{\mathrm{SF}}\right)$ and dynamic $\left(\Delta \mathrm{E}_{\mathrm{CT}-\mathrm{S} 1}\right)$ conditions of di-BODIPY in the gas phase for all 200 instantaneous configurations. (b) Probability distributions under different thermodynamics $\left(\Delta \mathrm{E}_{\mathrm{SF}}\right)$ and dynamic $\left(\Delta \mathrm{E}_{\mathrm{CT}-\mathrm{S} 1}\right)$ conditions in the gas phase.

\section{Electron Transitions of Excited States and Their Corresponding Frontier Molecular Orbitals of di-BODIPY for the AIMD Instantaneous Configurations and Four Possible Singlet Fission Channels in $\mathrm{CHCl}_{3}$}

Examinations reveal two channels for SF in di-BODIPY in $\mathrm{CHCl}_{3}$ : Channel A (including sub-Channel A1 and sub-Channel A2) and Channel B (including sub-Channel A1 and subChannel A2) which are simply described below. The main molecular orbitals for the considered transient geometric configurations are given in Table S10. Briefly, the same for these two channels (four sub-channels) are that they have effective driving force for $\mathrm{SF}$ (i.e. $\Delta \mathrm{E}_{\mathrm{SF}}$ $\leq 0$ ), and the main difference among them is which BODIPY moiety in di-BODIPY is excited. In sub-Channel A1/sub-Channel B1, the BODIPY(1) moiety is excited, generating the ${ }^{1} \mathrm{~S}_{1}$ excited state, and the representative transitions are given in Table S11 and S14. In sub-Channel A2/sub-Channel B2, the BODIPY(2) moiety is excited, generating the ${ }^{2} \mathrm{~S}_{1}$ excited state, and the representative transitions are given in Table S12 and S13. For sub-Channel A1 and subChannel A2, each channel is followed by a higher energy CT state (than the corresponding $\mathrm{S}_{1}$ 
state) which inhibits the SF proceedings, whereas for sub-Channel B1 and sub-Channel B2, each is followed by a low-energy CT state which can mediate the SF from the $\mathrm{S}_{1}$ state to the triplet pair state. Schematic illustration of SF of di-BODIPY in these four channels are given in Scheme S1 (sub-Channel A1), Scheme S2 (sub-Channel A2), Scheme S3 (sub-Channel B1), and Scheme S4 (sub-Channel B2), respectively.

\section{Summary for These Channels}

\begin{tabular}{|c|c|c|c|c|}
\hline \multicolumn{2}{|c|}{ Channels } & \multicolumn{3}{c|}{ Main characters } \\
\hline \multirow{2}{*}{ Channel A } & $\begin{array}{c}\text { Kinetic } \\
\text { barriers }\end{array}$ & $\begin{array}{c}\text { Thermodynamic } \\
\text { driving forces }\end{array}$ & $\begin{array}{c}\text { Open/closed } \\
\text { cases }\end{array}$ \\
\cline { 2 - 5 } & Sub-Channel A1 & $\Delta \mathrm{E}_{\mathrm{CT}-\mathrm{S} 1>0}>0$ & $\Delta \mathrm{E}_{\mathrm{SF}} \leq 0$ & Closed \\
\hline \multirow{3}{*}{ Channel B } & Sub-Channel A2 & $\Delta \mathrm{E}_{\mathrm{CT}-\mathrm{S} 1}>0$ & $\Delta \mathrm{E}_{\mathrm{SF}} \leq 0$ & Closed \\
\cline { 2 - 5 } & Sub-Channel B1 & $\Delta \mathrm{E}_{\mathrm{CT}-\mathrm{S} 1 \leq 0} \leq \mathrm{E}_{\mathrm{SF}} \leq 0$ & Open \\
\cline { 2 - 5 } & Sub-Channel B2 & $\Delta \mathrm{E}_{\mathrm{CT}-\mathrm{S} 1 \leq 0} \leq \mathrm{E}_{\mathrm{SF}} \leq 0$ & Open \\
\hline
\end{tabular}

Table S10. The Examples of Frontier Molecular Orbitals from HOMO to LUMO of di-BODIPY in $\mathrm{CHCl}_{3}$ for only 20 Instantaneous Configurations Extracted from a 2 ps AIMD Simulation Trajectory

\begin{tabular}{|c|c|c|c|c|}
\hline \multirow[b]{2}{*}{ Time/fs } & \multicolumn{2}{|c|}{ BODIPY(1) } & \multicolumn{2}{|c|}{ BODIPY(2) } \\
\hline & $\begin{array}{c}{ }^{1} \text { LUMO } \\
(308)\end{array}$ & $\begin{array}{c}{ }^{1} \mathrm{HOMO} \\
(306)\end{array}$ & $\begin{array}{c}{ }^{2} \text { LUMO } \\
(309)\end{array}$ & $\begin{array}{c}{ }^{2} \mathrm{HOMO} \\
(307)\end{array}$ \\
\hline 100 & 30 & $\because$ & 3 & 30 \\
\hline 200 & & & $\therefore$ & \\
\hline 300 & 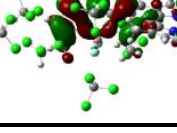 & & $\because+$ & 30 \\
\hline 400 & & & & \\
\hline
\end{tabular}




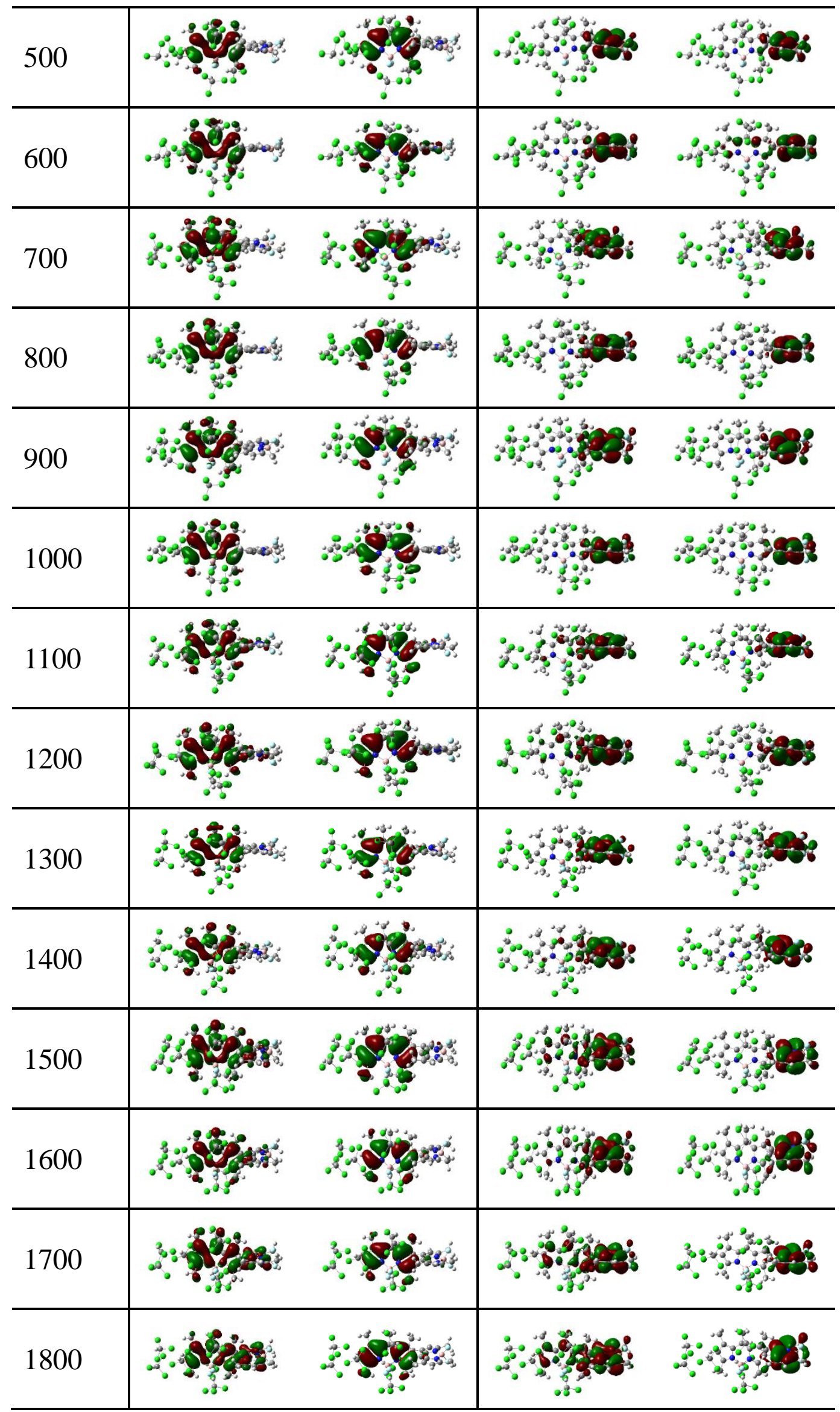




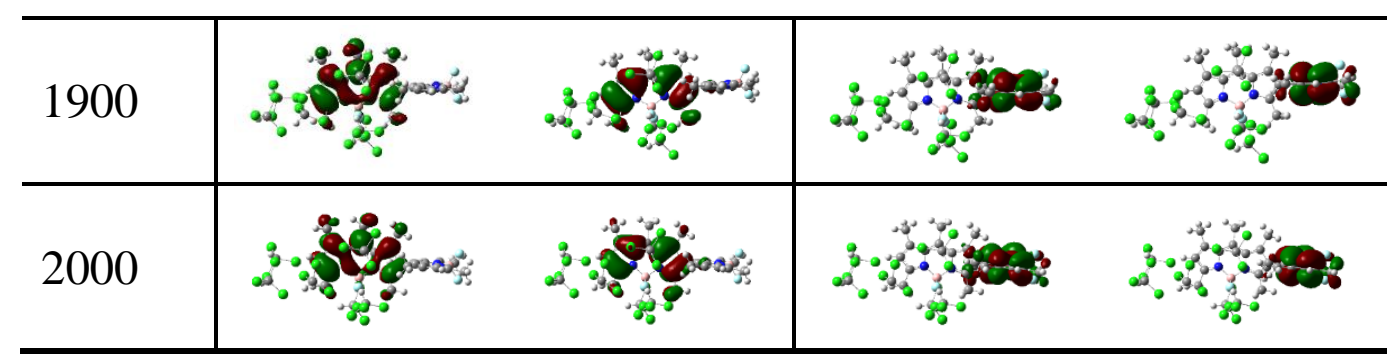

Note: $\overline{{ }^{1} \mathrm{HOMO} \text { and }{ }^{1} \text { LUMO represent the frontier molecular orbitals of BODIPY(1), while }{ }^{2} \mathrm{HOMO}}$ and ${ }^{2} \mathrm{LUMO}$ represent those of BODIPY(2).

\section{$\underline{\text { Sub-Channel A1 }}$}

Scheme S1. Schematic illustration of SF of di-BODIPY in sub-Channel A1. Photoexcitation Generates the Singlet excited state ${ }^{1} \mathrm{~S}_{1}$ (Table S11). A CT state is energetically higher than the singlet excited state and the singlet excited state cannot transition to the CT state. Thus, diBODIPY does not undergo SF in this channel. This is the case for di-BODIPY in the gas phase, apolar solvents (c-hex here) and even the weak polar solvents.

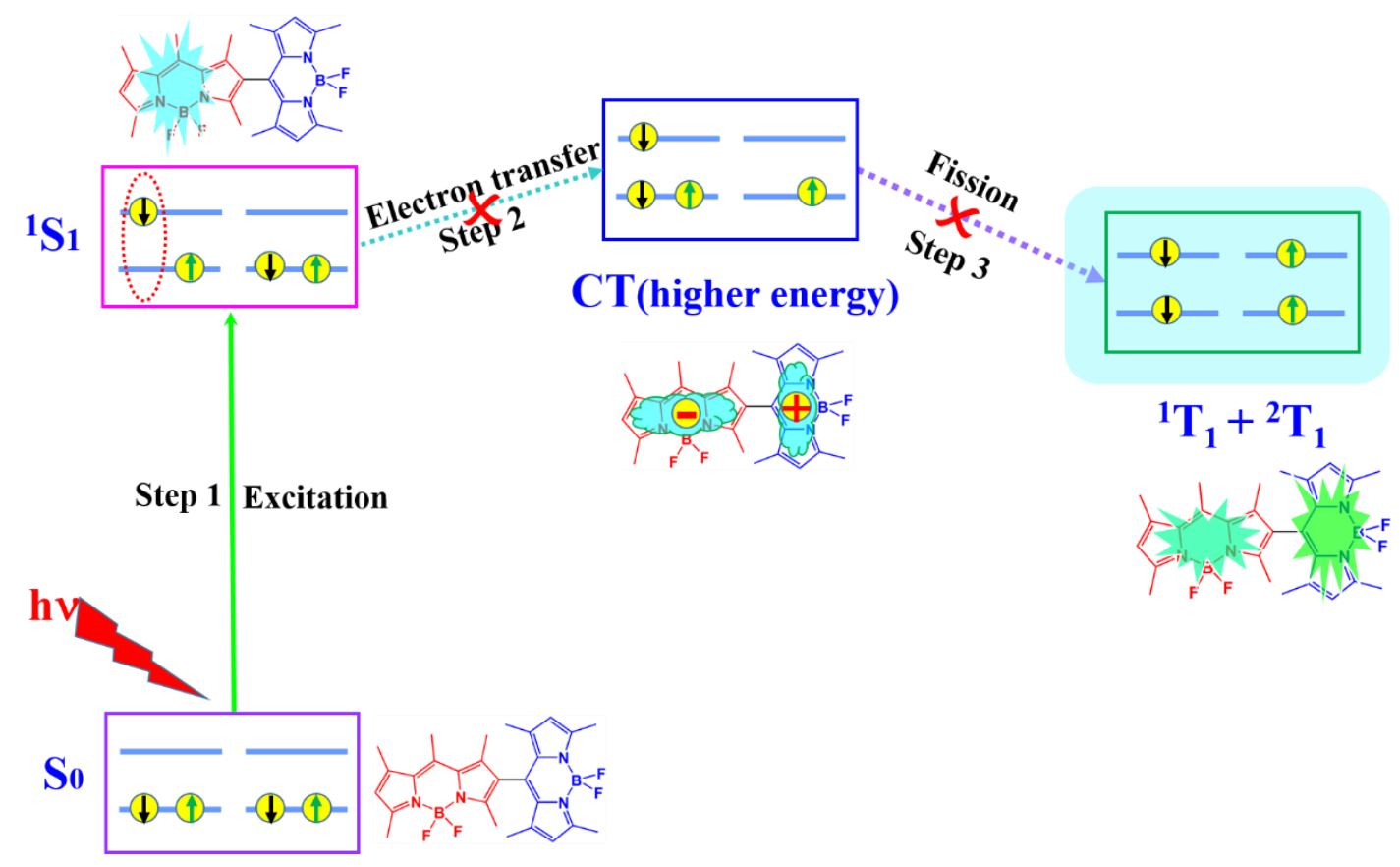


Table S11. The Example of Electron Transitions of Excited States and Their Corresponding Frontier Molecular Orbitals of di-BODIPY in $\mathrm{CHCl}_{3}$ with sub-Channel A1

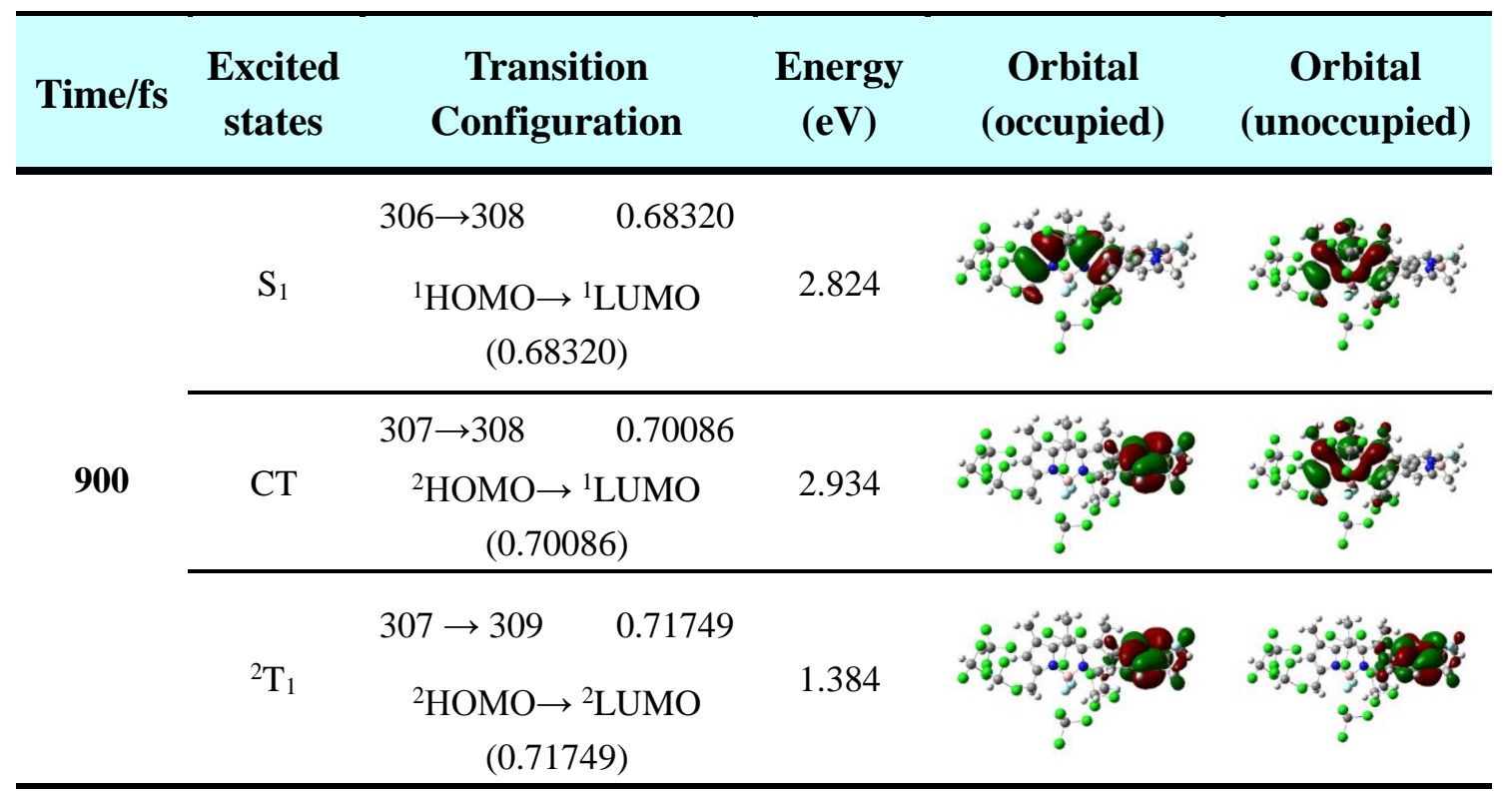

\section{Sub-Channel A2}

Scheme S2. Schematic illustration of SF of di-BODIPY in sub-Channel A2. Photoexcitation generates the singlet excited state ${ }^{2} \mathrm{~S}_{1}$ (Table S12). A CT state is energetically higher than the singlet excited state and the singlet excited state cannot transition to the CT state. Thus, diBODIPY does not undergo SF in this channel. This is also the case for di-BODIPY in the gas phase, apolar solvents (c-hex here) and even the weak polar solvents.

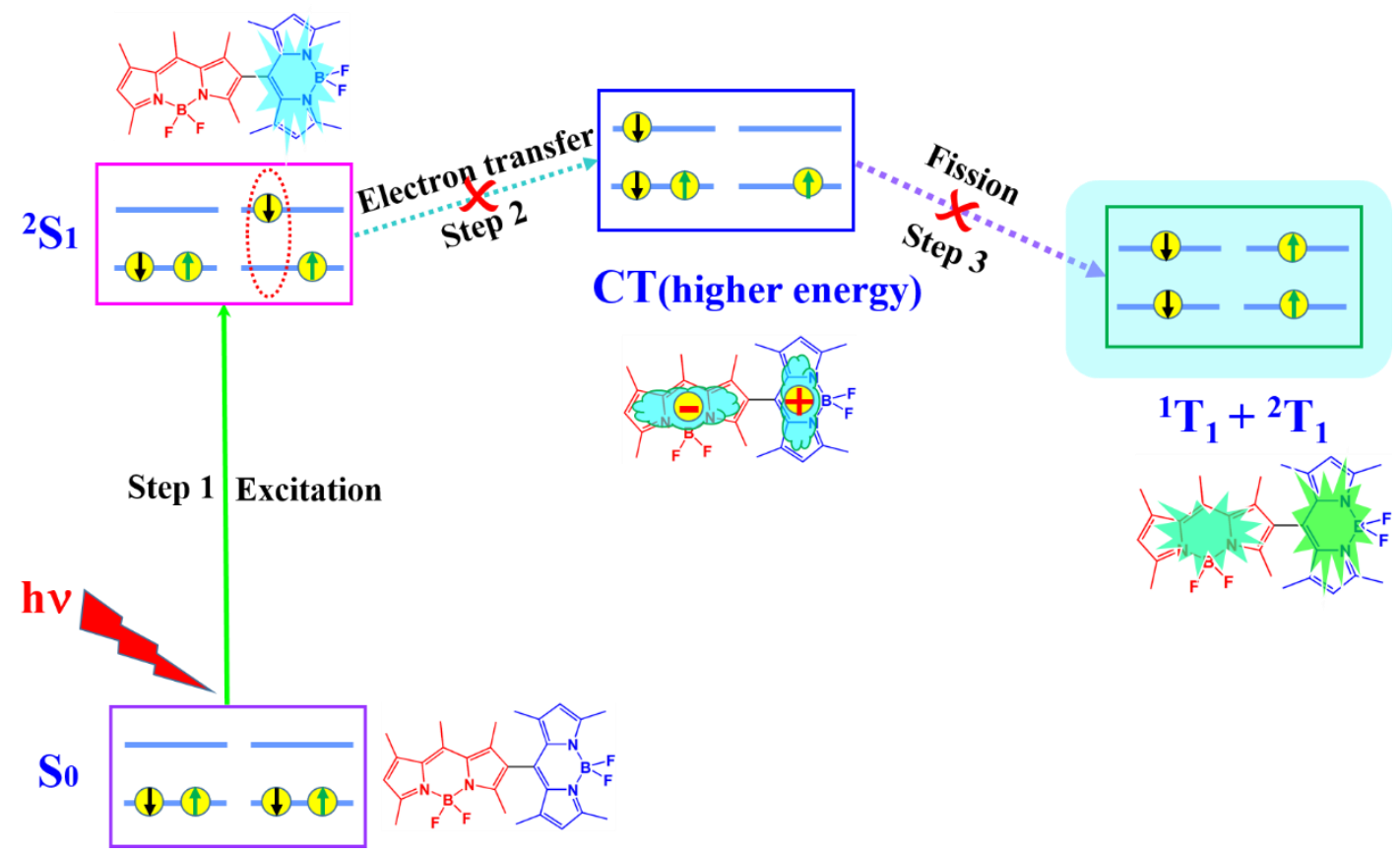


Table S12. The Examples of Electron Transitions of Excited States and Their Corresponding Frontier Molecular Orbitals of di-BODIPY in $\mathrm{CHCl}_{3}$ with sub-Channel $\mathbf{A 2}$

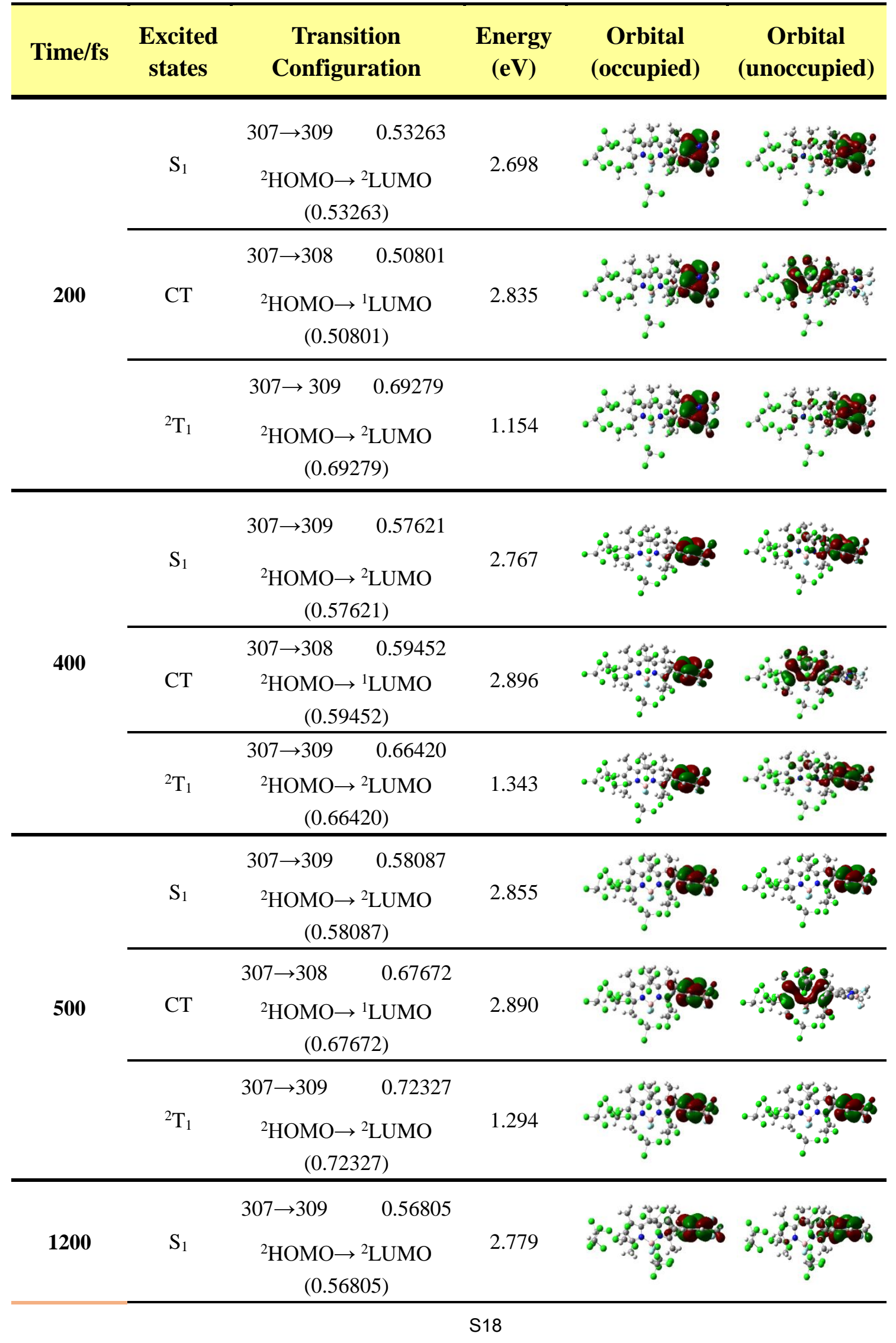




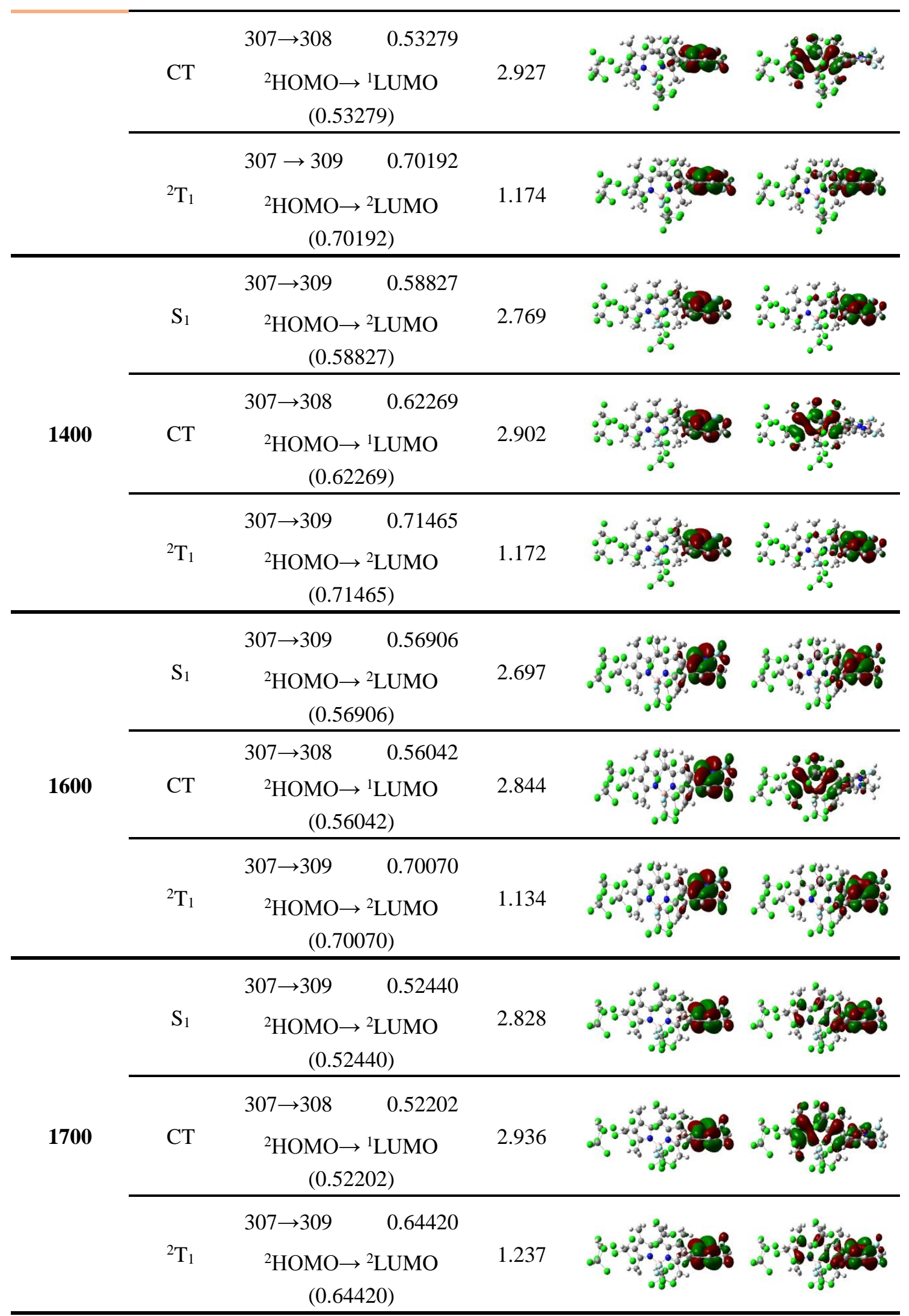




\section{Sub-Channel B2}

Scheme S3. Schematic illustration of SF of di-BODIPY through sub-Channel B2. Photoexcitation generates the singlet excited state ${ }^{2} \mathrm{~S}_{1}$ (Table S13), and a CT state is energetically lower than the singlet excited state, making the CT state an available intermediate state and providing a kinetic channel for the conversion of the singlet exciton to the CT state for the final triplet pair exciton. This is the case in $\mathrm{CHCl}_{3}$.

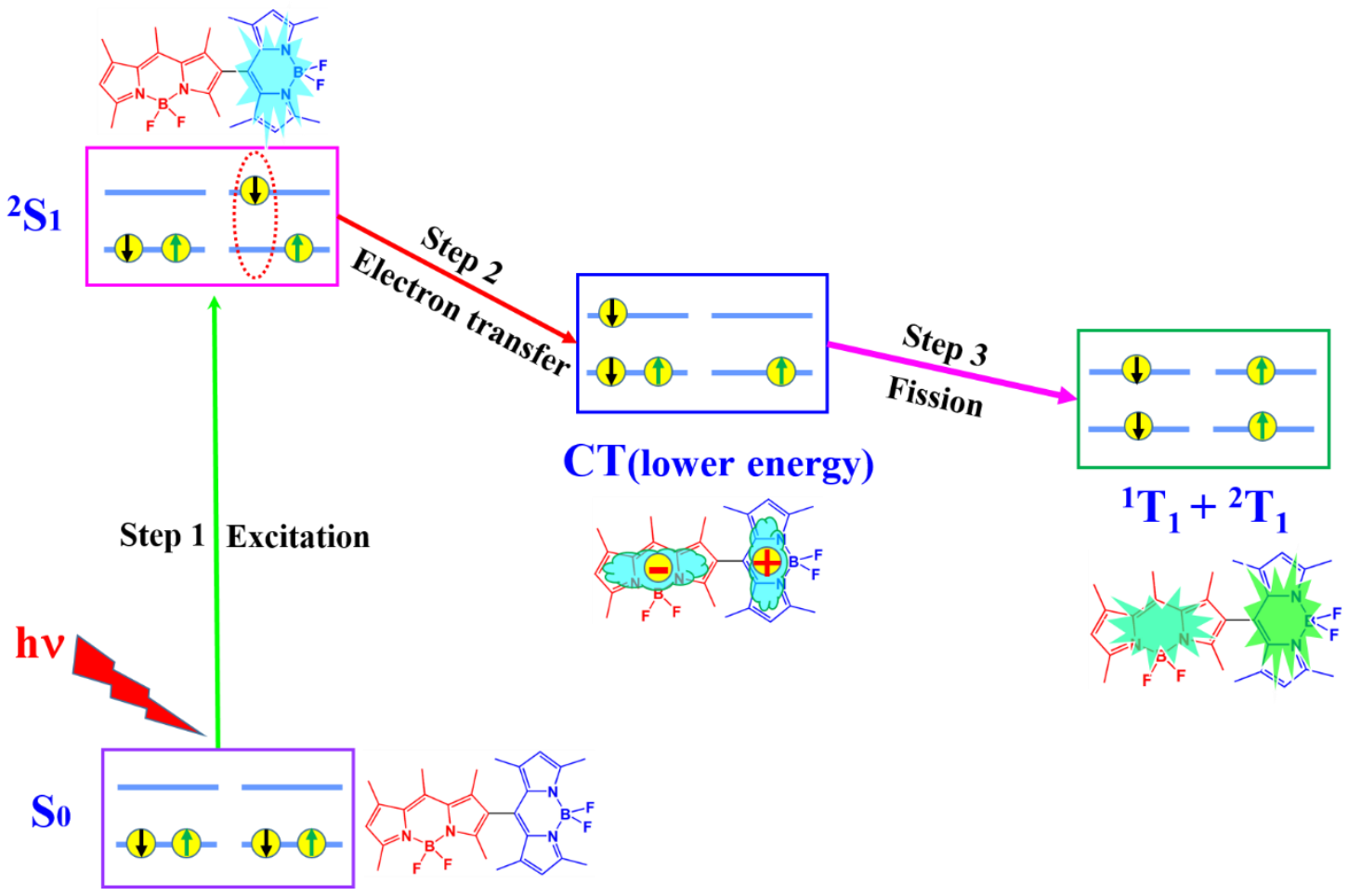

Table S13. The Examples of Electron Transitions of Excited States and Their Corresponding Frontier Molecular Orbitals of di-BODIPY in $\mathrm{CHCl}_{3}$ with sub-Channel $\mathbf{B 2}$

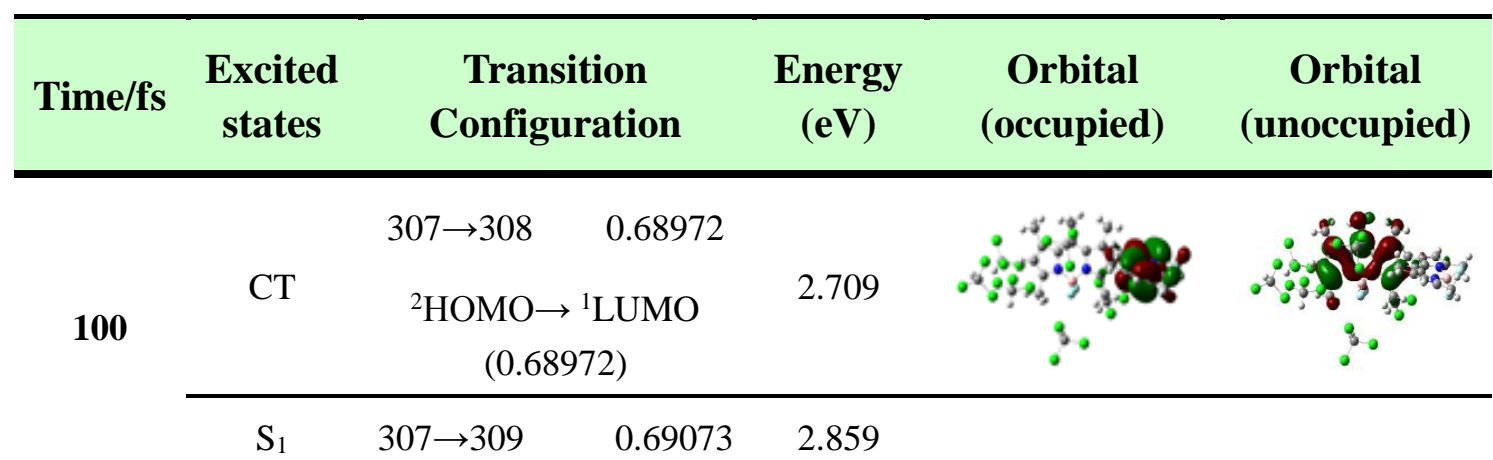




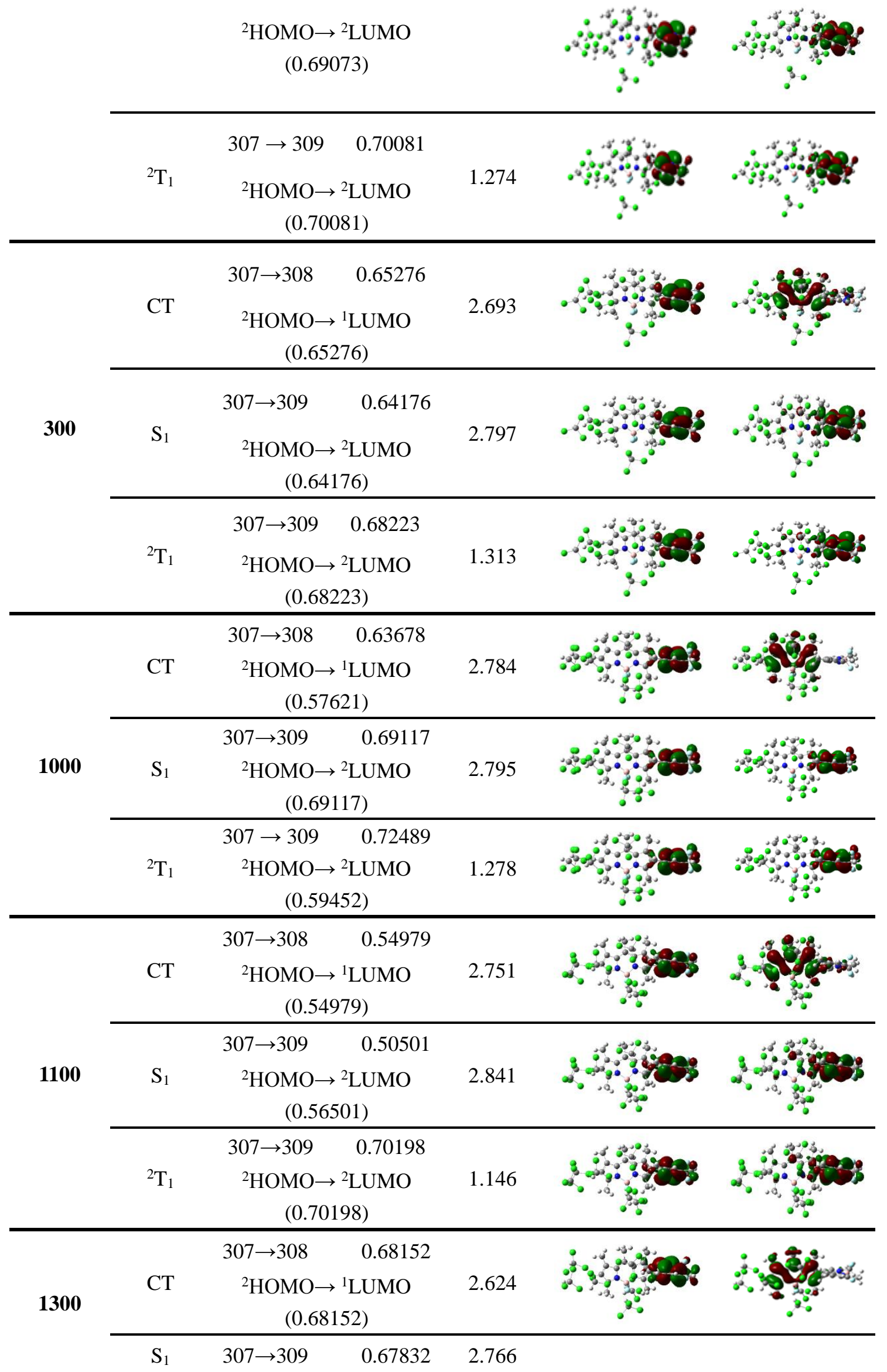




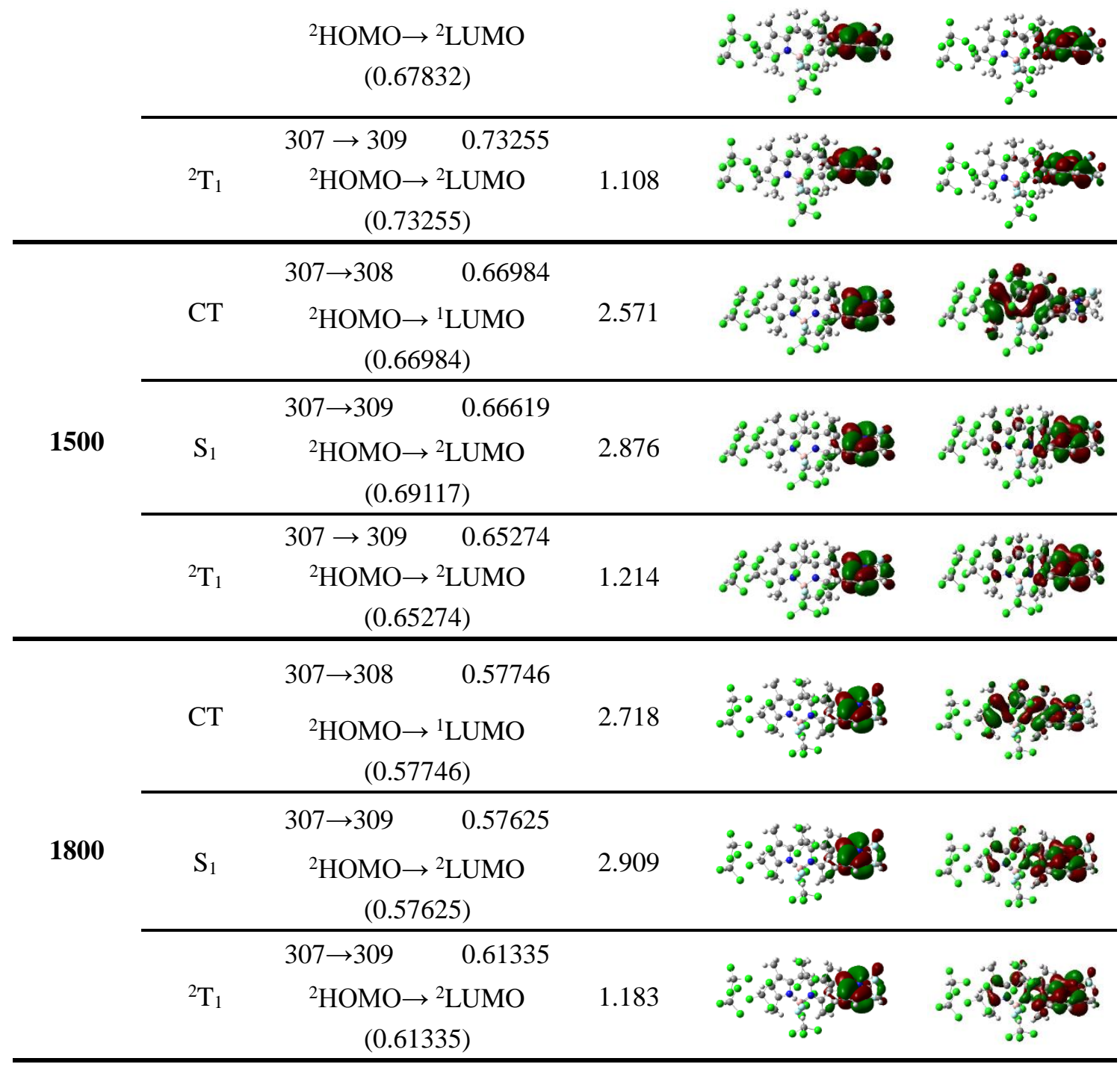

\section{Sub-Channel B1}

Scheme S4. Schematic illustration of SF of di-BODIPY in sub-Channel B1. Photoexcitation generates the singlet excited state ${ }^{1} \mathrm{~S}_{1}$ (Table S14), and a CT state is energetically lower than the singlet excited state, making the CT state an available intermediate state and providing a kinetic channel for the conversion of the singlet exciton to the CT state for the final triplet pair exciton. This is the case in $\mathrm{CHCl}_{3}$. 


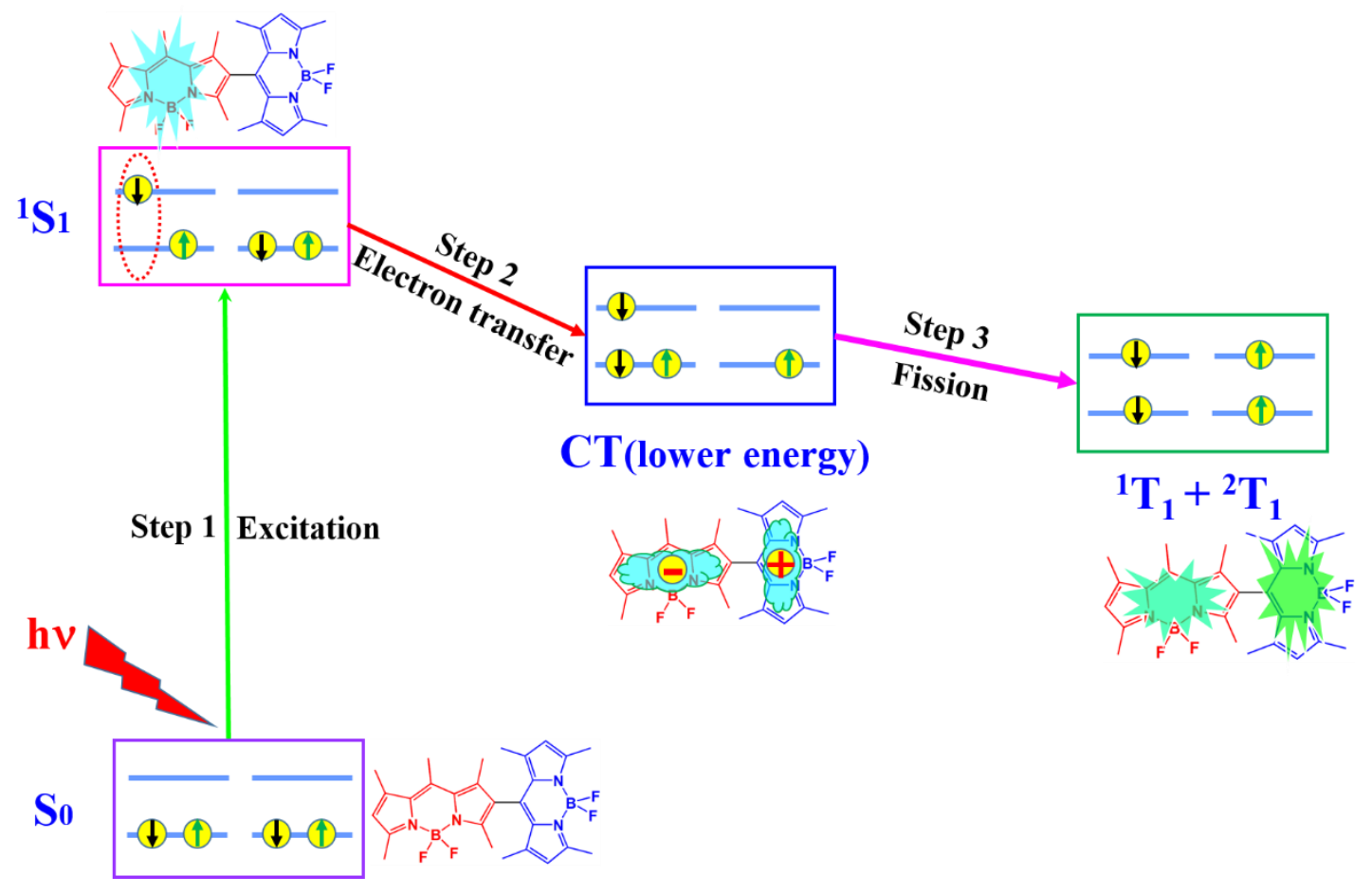

Table S14. The Example of Electron Transitions of Excited States and Their Corresponding Frontier Molecular Orbitals of di-BODIPY in $\mathrm{CHCl}_{3}$ with sub-Channel B1

\begin{tabular}{|c|c|c|c|c|c|}
\hline Time/fs & $\begin{array}{l}\text { Excited } \\
\text { states }\end{array}$ & $\begin{array}{c}\text { Transition } \\
\text { Configuration }\end{array}$ & $\begin{array}{c}\text { Energy } \\
(\mathrm{eV})\end{array}$ & $\begin{array}{c}\text { Orbital } \\
\text { (occupied) }\end{array}$ & $\begin{array}{c}\text { Orbital } \\
\text { (unoccupied) }\end{array}$ \\
\hline \multirow{5}{*}{450} & & $307 \rightarrow 308 \quad 0.55379$ & \multirow[b]{2}{*}{2.8162} & & \\
\hline & $\mathrm{CT}$ & $(0.55379)$ & & & \\
\hline & & $306 \rightarrow 308 \quad 0.60535$ & \multirow[b]{2}{*}{2.8294} & & \\
\hline & $S_{1}$ & $\begin{array}{c}{ }^{1} \mathrm{HOMO} \rightarrow{ }^{1} \mathrm{LUMO} \\
(0.70086) \\
\end{array}$ & & & \\
\hline & ${ }^{2} \mathrm{~T}_{1}$ & $\begin{array}{c}307 \rightarrow 309 \quad 0.69759 \\
{ }^{2} \mathrm{HOMO} \rightarrow{ }^{2} \mathrm{LUMO} \\
(0.69759)\end{array}$ & 1.3632 & & \\
\hline
\end{tabular}




\section{Electron Transitions of Excited States and Their Corresponding Frontier Molecular Orbitals of di-BODIPY for the AIMD Instantaneous Configurations and Two Possible Singlet Fission Channels in cyclohexane}

Examinations reveal a Channel A (including two sub-channels) for SF in di-BODIPY in cyclohexane: sub-Channel A1 and sub-Channel A2 which are simply described below. The main molecular orbitals for the considered transient geometric configurations are given in Table S15. Briefly, the main difference between the two channels is which BODIPY moiety in diBODIPY is excited. In sub-Channel A1, the BODIPY(1) moiety is excited, generating the ${ }^{1} \mathrm{~S}_{1}$ excited state, and the representative transitions are given in Table S16. In sub-Channel A2, the BODIPY(2) is excited, generating the ${ }^{2} S_{1}$ excited state, and the representative transitions are given in Table S17. Each channel is followed by a higher energy CT state (than the corresponding $\mathrm{S}_{1}$ state) which inhibits the SF proceedings. Schematic illustration of SF of diBODIPY in these two channels are given in Scheme S5 (sub-Channel A1) and Scheme S6 (subChannel A2), respectively.

Summary for The Channel A (including Two sub-Channels)

\begin{tabular}{|c|c|c|c|c|}
\hline \multicolumn{2}{|c|}{ Channels } & \multicolumn{3}{c|}{ Main characters } \\
\hline \multicolumn{2}{|c|}{} & Kinetic barriers & $\begin{array}{c}\text { Thermodynamic } \\
\text { driving force }\end{array}$ & $\begin{array}{c}\text { Open/closed } \\
\text { cases }\end{array}$ \\
\hline \multirow{3}{*}{ Channel A } & Sub-Channel A1 & $\Delta \mathrm{E}_{\mathrm{CT}-\mathrm{S} 1>0}>0$ & $\Delta \mathrm{E}_{\mathrm{SF}} \leq 0$ & Closed \\
\cline { 2 - 5 } & Sub-Channel A2 & $\Delta \mathrm{E}_{\mathrm{CT}-\mathrm{S} 1}>0$ & $\Delta \mathrm{E}_{\mathrm{SF}} \leq 0$ & Closed \\
\hline
\end{tabular}


Table S15. The Examples of Frontier Molecular Orbitals from HOMO to LUMO of di-BODIPY in c cyclohexane for only 20 Instantaneous Configurations Extracted from a 2 ps AIMD Simulation Trajectory

\begin{tabular}{|c|c|c|c|c|}
\hline \multirow[b]{2}{*}{ Time/fs } & \multicolumn{2}{|c|}{ BODIPY(1) } & \multicolumn{2}{|c|}{ BODIPY(2) } \\
\hline & $\begin{array}{c}{ }^{1} \text { LUMO } \\
(278)\end{array}$ & $\begin{array}{c}{ }^{1} \mathrm{HOMO} \\
(276)\end{array}$ & $\begin{array}{c}{ }^{2} \mathrm{LUMO} \\
(279)\end{array}$ & $\begin{array}{c}{ }^{2} \mathrm{HOMO} \\
(277)\end{array}$ \\
\hline 100 & & & & \\
\hline 200 & & & & \\
\hline 300 & & & 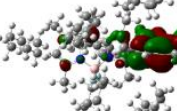 & \\
\hline 400 & & & & \\
\hline 500 & & & & \\
\hline 600 & & & & \\
\hline 700 & & & & \\
\hline 800 & & & 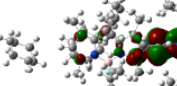 & \\
\hline 900 & & & 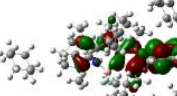 & 屬 \\
\hline 1000 & & & 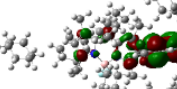 & \\
\hline 1100 & & & 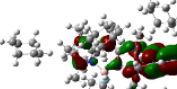 & 50 \\
\hline
\end{tabular}




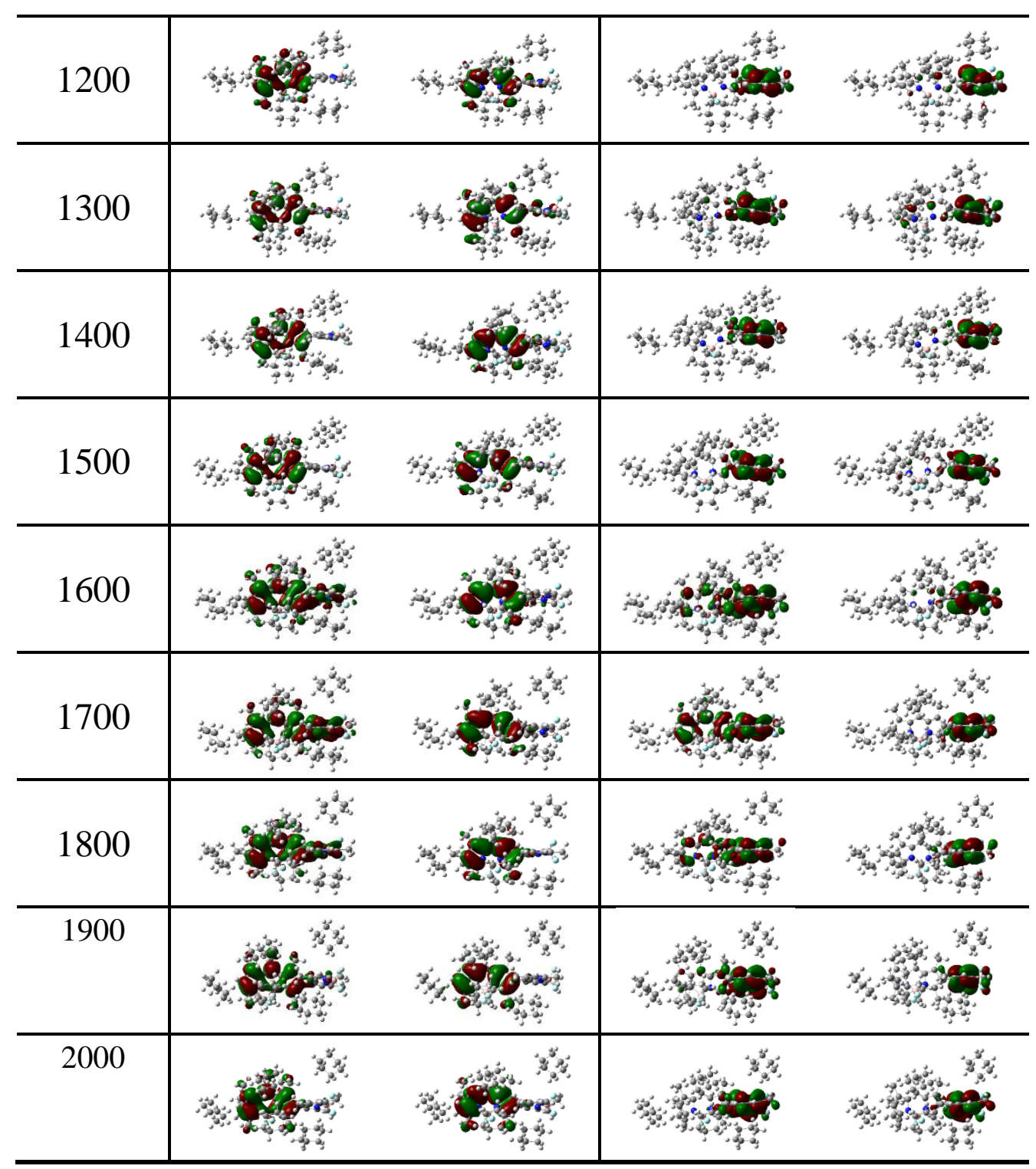




\section{Sub-Channel A1:}

Scheme S5. Schematic illustration of SF of di-BODIPY in sub-Channel A1. Photoexcitation generates the singlet excited state ${ }^{1} \mathrm{~S}_{1}$ (Table S16, exciting the BODIPY(1)). A CT state is energetically higher than the singlet excited state and the singlet excited states cannot transition to the CT state. Thus, di-BODIPY does not undergo SF in this channel.

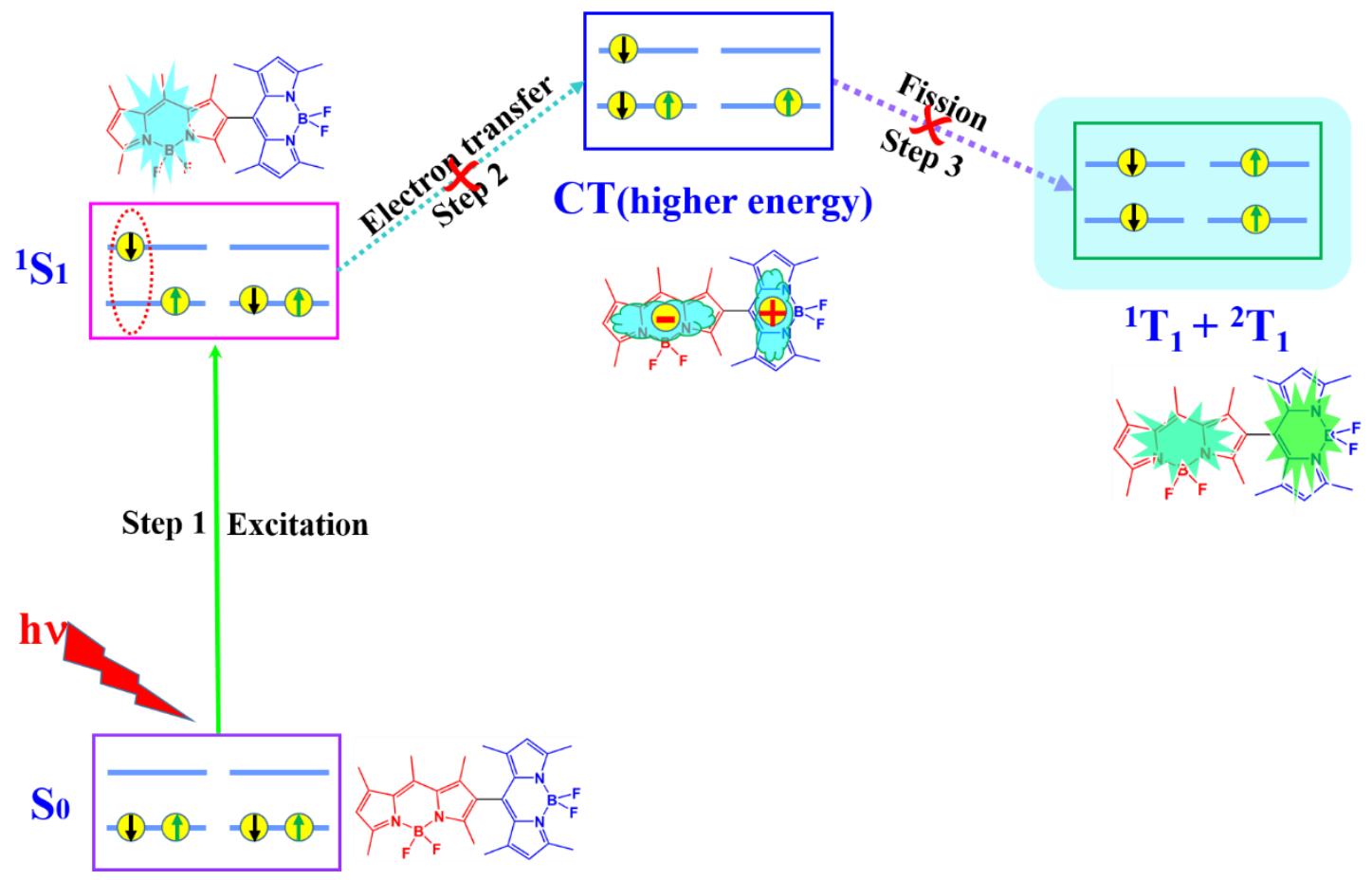

Table S16. The Examples of Electron Transitions of Excited States and Their Corresponding Frontier Molecular Orbitals of di-BODIPY in cyclohexane with sub-Channel A1

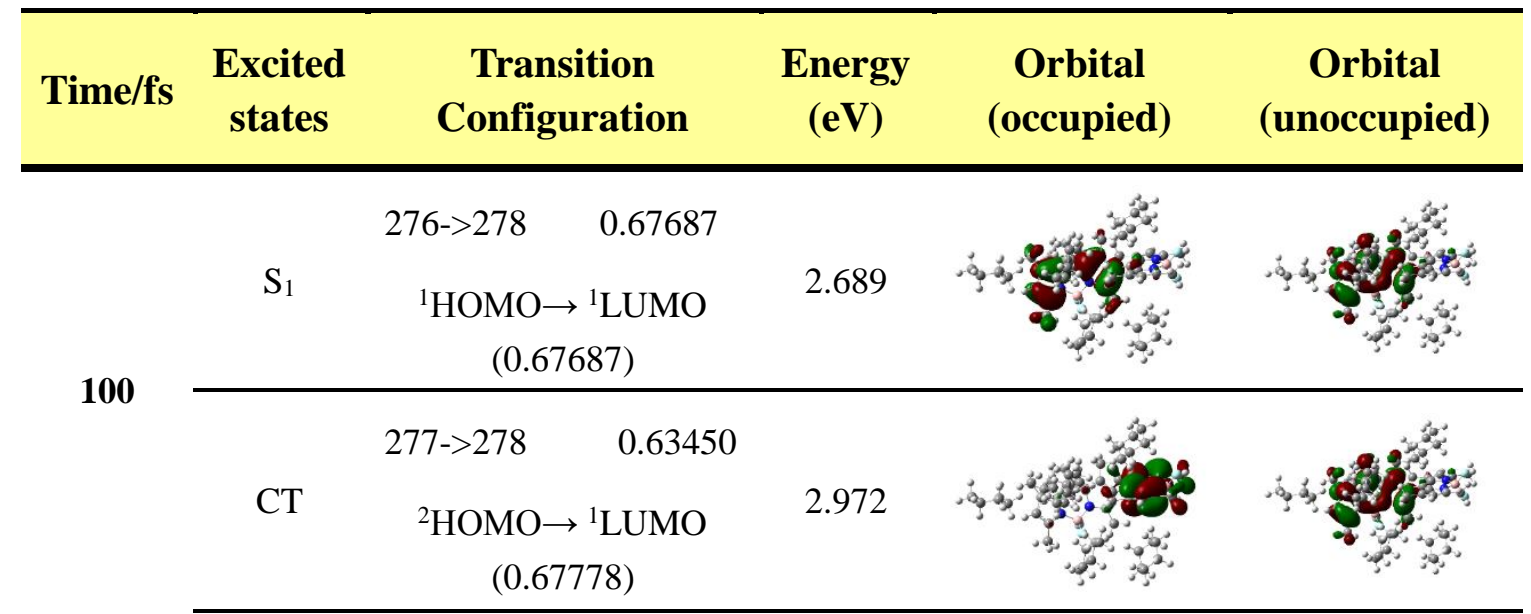




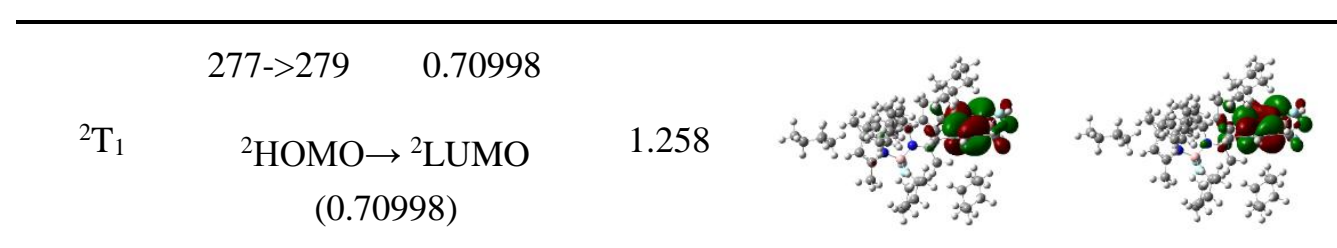

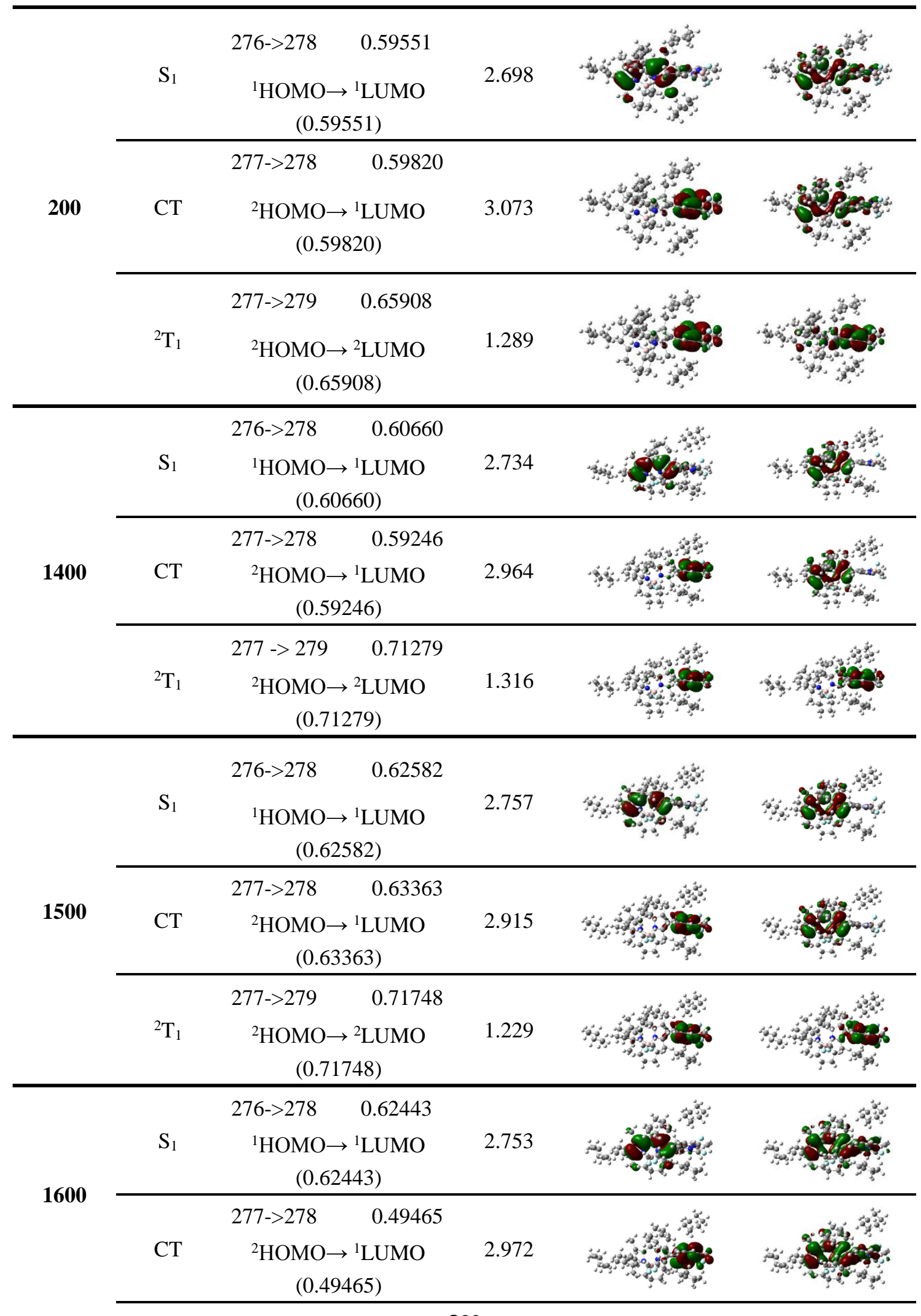




$\begin{array}{cccc}277->279 \quad 0.62676 & & \\ { }^{2} \mathrm{~T}_{1} & { }^{2} \mathrm{HOMO} \rightarrow{ }^{2} \mathrm{LUMO} & 1.353 & \\ & (0.62676) & & \end{array}$

\section{Sub-Channel A2:}

Scheme S6. Schematic illustration of SF of di-BODIPY in sub-Channel A2. Photoexcitation generates the singlet excited state ${ }^{2} \mathrm{~S}_{1}$ (Table S17, exciting the BODIPY(2)). A CT state is energetically higher than the singlet excited state and the singlet excited state cannot transition to the CT state. Thus, di-BODIPY does not undergo SF in this channel.

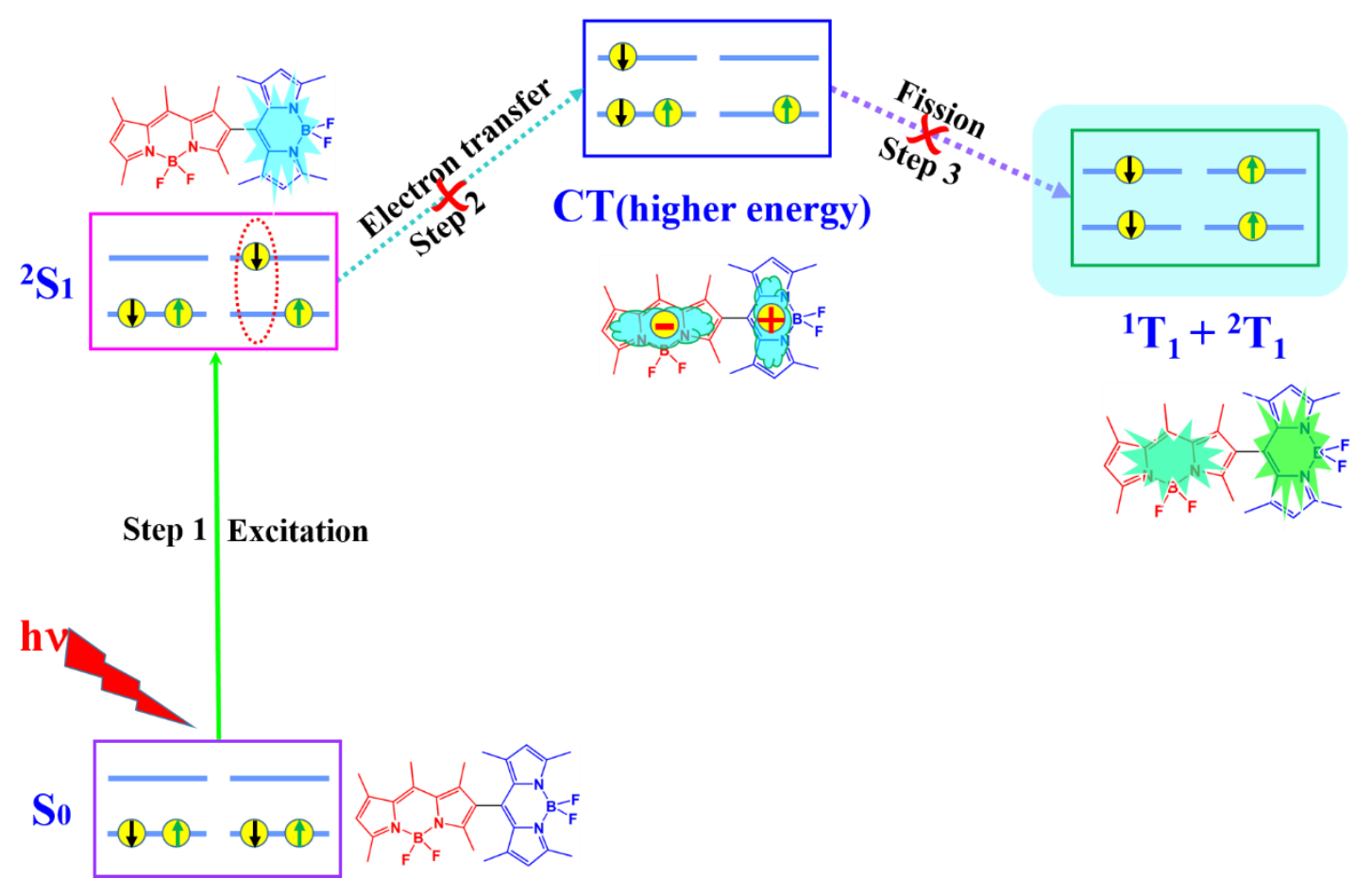


Table S17. The Example of Electron Transitions of Excited States and Their Corresponding Frontier Molecular Orbitals of di-BODIPY in cyclohexane with sub-Channel A2

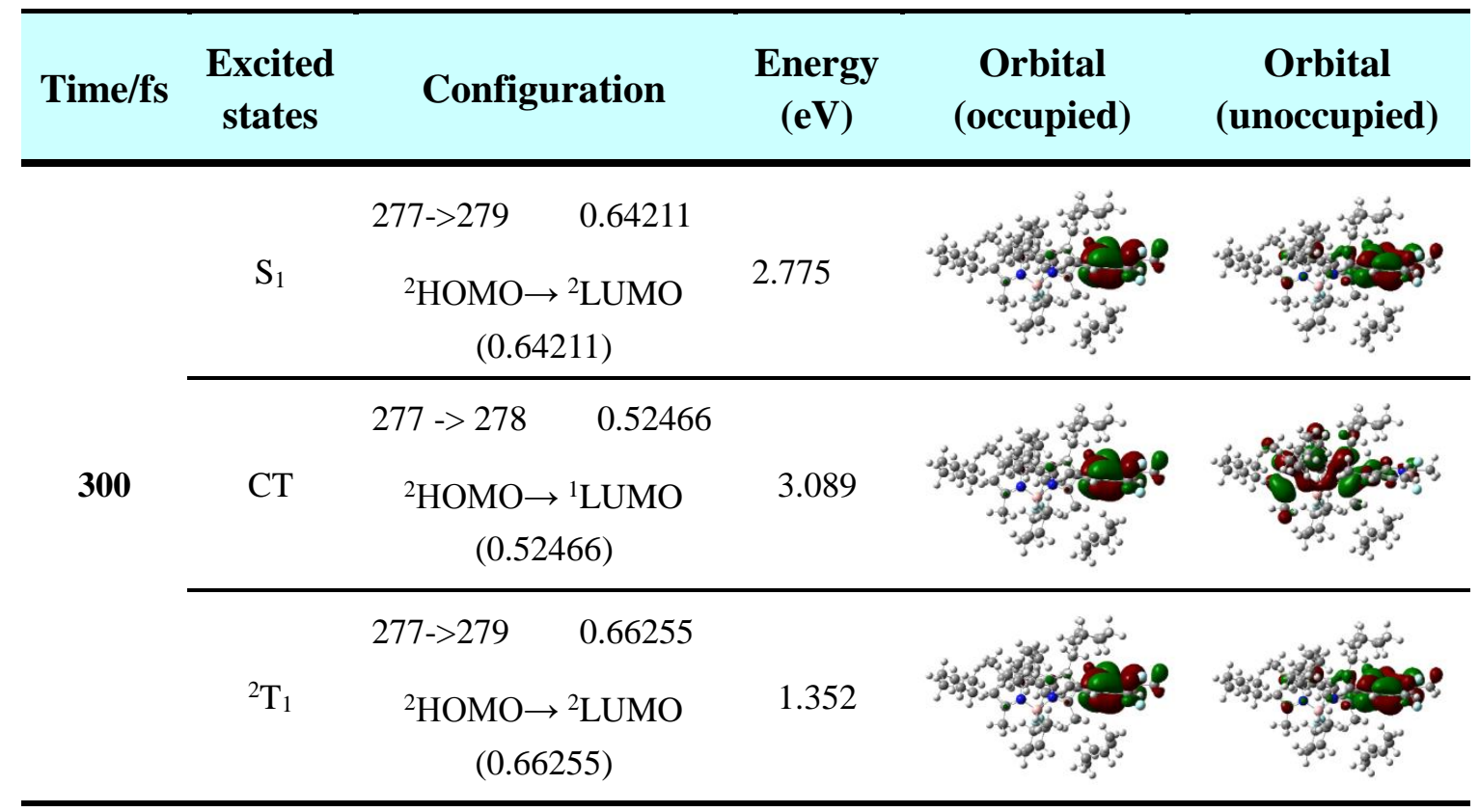

\section{Transition Configurations of the Smallest Vertical Excitation Energy of the} Triplet State

Table S18. The Smallest Triplet State Vertical Excitation Energies $\left(E\left({ }^{2} \mathrm{~T}_{1}\right)\right)$ and Corresponding Transitions of di-BODIPY for Partial Snapshot Configurations Which Enable $\mathrm{SF}$ in $\mathrm{CHCl}_{3}$

\begin{tabular}{c|c|cc|c|c|cc}
\hline $\begin{array}{c}\text { Times } \\
(\mathbf{f s})\end{array}$ & $\begin{array}{c}\mathbf{E}\left({ }^{2} \mathbf{T} \mathbf{1}\right) \\
(\mathbf{e V})\end{array}$ & \multicolumn{2}{|c|}{$\begin{array}{c}\text { Transition } \\
\text { Configurations }\end{array}$} & $\begin{array}{c}\text { Times } \\
(\mathbf{f s})\end{array}$ & $\begin{array}{c}\mathbf{E}\left({ }^{2} \mathbf{T}_{\mathbf{1}}\right) \\
(\mathbf{e V})\end{array}$ & \multicolumn{2}{c}{$\begin{array}{c}\text { Transition } \\
\text { Configurations }\end{array}$} \\
\hline 30 & 1.251 & $307->309$ & $60.28 \%$ & 980 & 1.323 & $307->309$ & $69.59 \%$ \\
50 & 1.261 & $307->309$ & $69.36 \%$ & 990 & 1.339 & $307->309$ & $71.67 \%$ \\
60 & 1.224 & $307->309$ & $68.30 \%$ & 1000 & 1.278 & $307->309$ & $72.49 \%$ \\
80 & 1.314 & $307->309$ & $71.57 \%$ & 1010 & 1.341 & $307->309$ & $71.95 \%$ \\
100 & 1.274 & $307->309$ & $70.08 \%$ & 1020 & 1.409 & $307->309$ & $69.28 \%$ \\
110 & 1.301 & $307->309$ & $65.63 \%$ & 1040 & 1.339 & $307->309$ & $72.23 \%$ \\
130 & 1.043 & $307->309$ & $62.99 \%$ & 1050 & 1.300 & $307->309$ & $71.52 \%$ \\
150 & 1.297 & $307->309$ & $67.07 \%$ & 1070 & 1.205 & $307->309$ & $70.80 \%$ \\
180 & 1.258 & $307->309$ & $71.63 \%$ & 1100 & 1.174 & $307->309$ & $70.19 \%$ \\
200 & 1.154 & $307->309$ & $69.28 \%$ & 1140 & 1.315 & $307->309$ & $66.16 \%$
\end{tabular}




\begin{tabular}{|c|c|c|c|c|c|c|c|}
\hline 230 & 1.162 & $307->309$ & $63.58 \%$ & 1170 & 1.206 & $307->309$ & $69.29 \%$ \\
\hline 260 & 1.252 & 307 -> 309 & $71.41 \%$ & 1190 & 1.296 & $307->309$ & $72.02 \%$ \\
\hline 280 & 1.276 & 307 -> 309 & $70.33 \%$ & 1200 & 1.146 & $307->309$ & $70.20 \%$ \\
\hline 300 & 1.313 & 307 -> 309 & $68.22 \%$ & 1220 & 1.303 & $307->309$ & $68.15 \%$ \\
\hline 330 & 1.161 & 307 -> 309 & $72.56 \%$ & 1230 & 1.356 & $307->309$ & $67.99 \%$ \\
\hline 350 & 1.299 & 307 -> 309 & $71.32 \%$ & 1260 & 1.297 & $307->309$ & $72.08 \%$ \\
\hline 360 & 1.145 & 307 -> 309 & $72.86 \%$ & 1290 & 1.257 & $307->309$ & $70.89 \%$ \\
\hline 370 & 1.382 & 307 -> 309 & $70.25 \%$ & 1300 & 1.037 & $307->309$ & $73.26 \%$ \\
\hline 380 & 1.254 & 307 -> 309 & $69.80 \%$ & 1350 & 1.296 & $307->309$ & $70.05 \%$ \\
\hline 390 & 1.360 & 307 -> 309 & $70.64 \%$ & 1360 & 1.298 & $307->309$ & $68.07 \%$ \\
\hline 400 & 1.343 & 307 -> 309 & $66.42 \%$ & 1390 & 1.343 & $307->309$ & $66.68 \%$ \\
\hline 410 & 1.257 & 307 -> 309 & $72.14 \%$ & 1400 & 1.172 & $307->309$ & $71.47 \%$ \\
\hline 430 & 1.290 & $307->309$ & $69.87 \%$ & 1410 & 1.319 & $307->309$ & $60.94 \%$ \\
\hline 440 & 1.324 & 307 -> 309 & $70.76 \%$ & 1420 & 1.294 & $307->309$ & $67.89 \%$ \\
\hline 450 & 1.363 & 307 -> 309 & $51.05 \%$ & 1450 & 1.098 & $307->309$ & $65.96 \%$ \\
\hline 460 & 1.288 & 307 -> 309 & $70.41 \%$ & 1480 & 1.213 & $307->309$ & $62.45 \%$ \\
\hline 500 & 1.294 & $307->309$ & $72.33 \%$ & 1500 & 1.214 & $307->309$ & $65.55 \%$ \\
\hline 510 & 1.087 & 307 -> 309 & $73.70 \%$ & 1510 & 1.156 & $307->309$ & $64.82 \%$ \\
\hline 530 & 1.319 & 307 -> 309 & $72.37 \%$ & 1550 & 1.332 & $307->309$ & $71.15 \%$ \\
\hline 540 & 1.277 & 307 -> 309 & $71.11 \%$ & 1580 & 1.005 & $307->309$ & $71.75 \%$ \\
\hline 560 & 1.265 & 307 -> 309 & $71.53 \%$ & 1600 & 1.134 & $307->309$ & $70.07 \%$ \\
\hline 590 & 1.272 & 307 -> 309 & $71.96 \%$ & 1610 & 1.203 & $307->309$ & $60.04 \%$ \\
\hline 640 & 1.285 & 307 -> 309 & $70.99 \%$ & 1650 & 1.396 & $307->309$ & $67.36 \%$ \\
\hline 660 & 1.149 & 307 -> 309 & $71.83 \%$ & 1670 & 1.179 & $307->309$ & $58.33 \%$ \\
\hline 670 & 1.241 & $307->309$ & $62.07 \%$ & 1680 & 1.305 & $307->309$ & $68.02 \%$ \\
\hline 690 & 1.220 & 307 -> 309 & $71.58 \%$ & 1700 & 1.237 & $307->309$ & $64.42 \%$ \\
\hline 720 & 1.364 & 307 -> 309 & $70.72 \%$ & 1710 & 1.334 & $307->309$ & $63.50 \%$ \\
\hline 730 & 1.428 & 307 -> 309 & $71.75 \%$ & 1760 & 1.260 & $307->309$ & $69.79 \%$ \\
\hline 740 & 1.329 & 307 -> 309 & $70.49 \%$ & 1780 & 1.356 & $307->309$ & $69.65 \%$ \\
\hline 760 & 1.274 & 307 -> 309 & $70.58 \%$ & 1800 & 1.183 & $307->309$ & $61.34 \%$ \\
\hline 790 & 1.262 & 307 -> 309 & $85.57 \%$ & 1830 & 1.295 & $307->309$ & $62.93 \%$ \\
\hline 820 & 1.275 & 307 -> 309 & $71.79 \%$ & 1860 & 1.192 & $307->309$ & $69.16 \%$ \\
\hline 850 & 1.371 & 307 -> 309 & $71.90 \%$ & 1890 & 1.238 & $307->309$ & $70.71 \%$ \\
\hline 890 & 1.244 & 307 -> 309 & $68.98 \%$ & 1910 & 1.338 & $307->309$ & $66.43 \%$ \\
\hline 910 & 1.414 & 307 -> 309 & $70.79 \%$ & 1930 & 1.326 & $307->309$ & $66.94 \%$ \\
\hline 920 & 1.192 & 307 -> 309 & $72.80 \%$ & 1960 & 1.047 & 307 -> 309 & $71.90 \%$ \\
\hline 940 & 1.343 & 307 -> 309 & $71.99 \%$ & 1990 & 1.311 & $307->309$ & $71.86 \%$ \\
\hline 950 & 1.235 & 307 -> 309 & $69.68 \%$ & & & & \\
\hline
\end{tabular}


Table S19. The Smallest Triplet State Vertical Excitation Energies $\left(E\left({ }^{2} \mathrm{~T}_{1}\right)\right)$ and Corresponding Transitions of di-BODIPY for Partial Snapshot Configurations Which Enable SF in cyclohexane

\begin{tabular}{|c|c|c|c|c|c|c|c|}
\hline \multirow{2}{*}{$\begin{array}{c}\begin{array}{c}\text { Times } \\
\text { (fs) }\end{array} \\
40\end{array}$} & \multirow{2}{*}{$\begin{array}{c}\mathbf{E}\left({ }^{2} \mathbf{T}_{\mathbf{1}}\right) \\
(\mathbf{e V}) \\
1.170\end{array}$} & \multicolumn{2}{|c|}{$\begin{array}{c}\text { Transition } \\
\text { Configurations }\end{array}$} & \multirow{2}{*}{$\begin{array}{c}\begin{array}{c}\text { Times } \\
(\mathbf{f s})\end{array} \\
1120\end{array}$} & \multirow{2}{*}{$\begin{array}{c}\mathbf{E}\left({ }^{2} \mathbf{T}_{1}\right) \\
(\mathbf{e V})\end{array}$} & \multicolumn{2}{|c|}{$\begin{array}{c}\text { Transition } \\
\text { Configurations }\end{array}$} \\
\hline & & $277->279$ & $72.42 \%$ & & & 277 -> 279 & $57.18 \%$ \\
\hline 70 & 1.335 & $277->279$ & $72.23 \%$ & 1140 & 1.189 & 277 -> 279 & $67.57 \%$ \\
\hline 100 & 1.258 & 277 -> 279 & $70.99 \%$ & 1180 & 1.331 & 277 -> 279 & $72.01 \%$ \\
\hline 130 & 1.276 & $277->279$ & $57.78 \%$ & 1190 & 1.207 & 277 -> 279 & $65.51 \%$ \\
\hline 150 & 1.250 & $277->279$ & $71.46 \%$ & 1210 & 1.205 & 277 -> 279 & $65.56 \%$ \\
\hline 160 & 1.319 & 277 -> 279 & $71.27 \%$ & 1220 & 1.251 & 277 -> 279 & $69.37 \%$ \\
\hline 200 & 1.289 & $277->279$ & $65.91 \%$ & 1240 & 1.104 & $277->279$ & $52.02 \%$ \\
\hline 230 & 1.225 & $277->279$ & $72.49 \%$ & 1250 & 1.315 & $277->279$ & $51.70 \%$ \\
\hline 250 & 1.349 & 277 -> 279 & $64.27 \%$ & 1270 & 1.254 & 277 -> 279 & $70.94 \%$ \\
\hline 260 & 1.218 & 277 -> 279 & $71.62 \%$ & 1280 & 1.331 & 277 -> 279 & $67.34 \%$ \\
\hline 280 & 1.252 & 277 -> 279 & $70.38 \%$ & 1290 & 1.308 & 277 -> 279 & $61.49 \%$ \\
\hline 300 & 1.352 & 277 -> 279 & $66.26 \%$ & 1310 & 1.254 & 277 -> 279 & $68.92 \%$ \\
\hline 330 & 1.0630 & 277 -> 279 & $71.05 \%$ & 1340 & 1.268 & 277 -> 279 & $71.85 \%$ \\
\hline 360 & 1.190 & 277 -> 279 & $68.94 \%$ & 1370 & 1.166 & 277 -> 279 & $72.52 \%$ \\
\hline 410 & 1.268 & $277->279$ & $71.51 \%$ & 1390 & 1.366 & $277->279$ & $66.97 \%$ \\
\hline 430 & 1.255 & 277 -> 279 & $72.18 \%$ & 1400 & 1.316 & 277 -> 279 & $71.28 \%$ \\
\hline 450 & 1.299 & $277->279$ & $71.98 \%$ & 1410 & 1.192 & $277->279$ & $50.63 \%$ \\
\hline 480 & 1.294 & $277->279$ & $71.05 \%$ & 1430 & 1.362 & $277->279$ & $59.13 \%$ \\
\hline 510 & 1.130 & $277->279$ & $61.73 \%$ & 1440 & 1.317 & $277->279$ & $61.27 \%$ \\
\hline 550 & 1.406 & 277 -> 279 & $71.40 \%$ & 1460 & 1.238 & 277 -> 279 & $52.82 \%$ \\
\hline 570 & 1.341 & $277->279$ & $63.47 \%$ & 1480 & 1.318 & $277->279$ & $63.07 \%$ \\
\hline 580 & 1.169 & 277 -> 279 & $72.20 \%$ & 1500 & 1.229 & 277 -> 279 & $71.75 \%$ \\
\hline 610 & 1.196 & 277 -> 279 & $71.83 \%$ & 1530 & 1.129 & 277 -> 279 & $67.26 \%$ \\
\hline 640 & 1.187 & 277 -> 279 & $71.64 \%$ & 1560 & 1.276 & 277 -> 279 & $69.21 \%$ \\
\hline 660 & 1.328 & $277->279$ & $71.62 \%$ & 1600 & 1.353 & $277->279$ & $62.68 \%$ \\
\hline 670 & 1.242 & 277 -> 279 & $70.05 \%$ & 1630 & 1.104 & 277 -> 279 & $60.59 \%$ \\
\hline 680 & 1.244 & 277 -> 279 & $71.54 \%$ & 1660 & 1.244 & 277 -> 279 & $70.41 \%$ \\
\hline 690 & 1.391 & 277 -> 279 & $71.66 \%$ & 1680 & 1.391 & 277 -> 279 & $51.67 \%$ \\
\hline 710 & 1.304 & 277 -> 279 & $71.92 \%$ & 1700 & 1.343 & 277 -> 279 & $59.23 \%$ \\
\hline 740 & 1.110 & 277 -> 279 & $65.24 \%$ & 1710 & 1.321 & 277 -> 279 & $61.43 \%$ \\
\hline 790 & 1.240 & 277 -> 279 & $54.74 \%$ & 1730 & 1.365 & 277 -> 279 & $69.30 \%$ \\
\hline 860 & 1.319 & 277 -> 279 & $71.72 \%$ & 1760 & 1.309 & 277 -> 279 & $72.49 \%$ \\
\hline
\end{tabular}




\begin{tabular}{c|l|ll|l|l|ll}
890 & 1.263 & $277->279$ & $67.23 \%$ & 1780 & 1.359 & 277 -> 279 & $70.05 \%$ \\
920 & 1.280 & $277->279$ & $57.40 \%$ & 1810 & 1.385 & $277->279$ & $68.34 \%$ \\
960 & 1.220 & $277->279$ & $68.41 \%$ & 1860 & 1.319 & $277->279$ & $70.98 \%$ \\
970 & 1.294 & $277->279$ & $48.48 \%$ & 1880 & 1.175 & $277->279$ & $54.89 \%$ \\
990 & 1.139 & $277->279$ & $55.45 \%$ & 1910 & 1.298 & $277->279$ & $71.45 \%$ \\
1020 & 1.243 & $277->279$ & $60.37 \%$ & 1940 & 1.335 & $277->279$ & $66.89 \%$ \\
1070 & 1.342 & $277->279$ & $71.34 \%$ & 1950 & 1.132 & $277->279$ & $68.69 \%$ \\
1090 & 1.226 & $277->279$ & $70.82 \%$ & 1980 & 1.108 & $277->279$ & $71.13 \%$ \\
\hline
\end{tabular}

\section{Orbital Energy Correlation and Time Evolution Dynamics and Relationship between the CT State Energies and ${ }^{1}$ LUMO Energies}

The molecular orbital energy correlation and time evolution dynamics are displayed in Figure S6. The relationships between the ${ }^{1}$ LUMO energy and the CT state energy is illustrated in Figure S7, displaying the CT state energies and ${ }^{1}$ LUMO energies with a quasilinear correlation between them. No matter in $\mathrm{CHCl}_{3}$ or cyclohexane, the $\mathrm{CT}$ state energy increases gradually with arising of the ${ }^{1}$ LUMO energy. Clearly, the CT state energy is basically determined by the ${ }^{1}$ LUMO energy in both solvents. The low correlativity should be attributed to the fact that the CT occurs between two molecular fragments which have different changes in structural fluctuation of di-BODIPY and thus different electronic property changes.
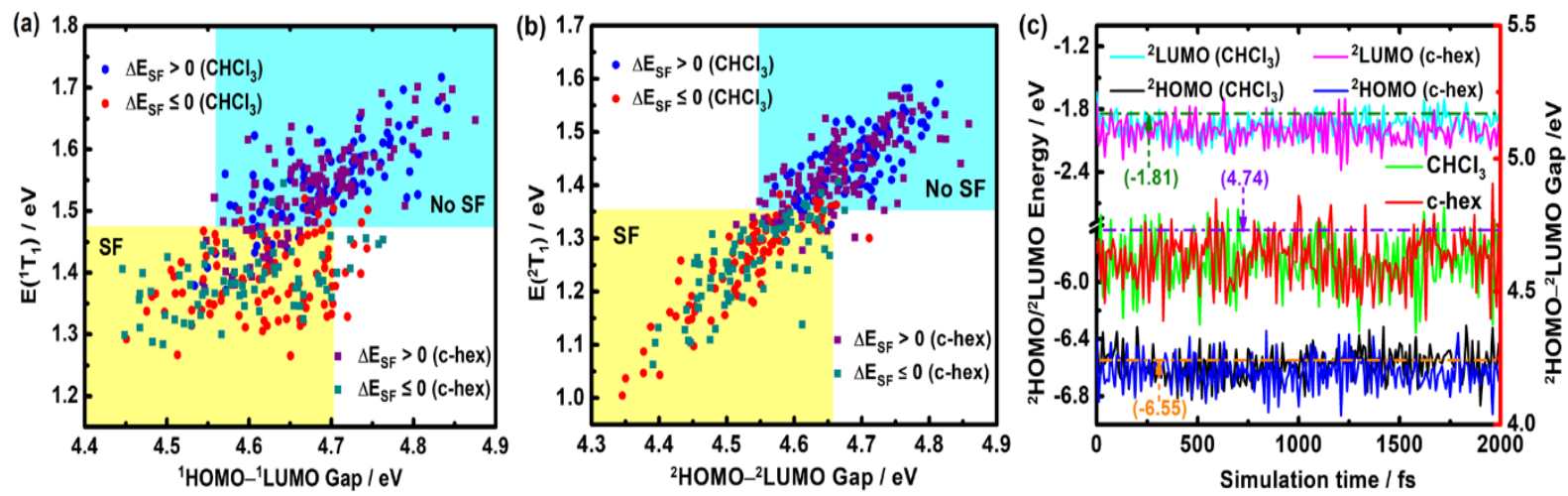

Figure S6. Orbital energy correlation and time evolution dynamics.

(a) The relationship between the ${ }^{1} \mathrm{HOMO}-{ }^{1}$ LUMO energy gap and smallest vertical local excitation energy of triplet state $\left(\mathrm{E}\left({ }^{1} \mathrm{~T}_{1}\right)\right)$. (b) The relationship between the ${ }^{2} \mathrm{HOMO}-{ }^{2} \mathrm{LUMO}$ energy gap and smallest 
vertical local excitation energy of triplet state $\left(E\left({ }^{2} \mathrm{~T}_{1}\right)\right)$. The red dots $\left(\Delta \mathrm{E}_{\mathrm{SF}} \leq 0\right)$ denote that diBODIPY meets the SF energy criterion in $\mathrm{CHCl}_{3}$ and the blue dots $\left(\Delta \mathrm{E}_{\mathrm{SF}}>0\right)$ denote that it doesn't in $\mathrm{CHCl}_{3}$. The dark cyan dots $\left(\Delta \mathrm{E}_{\mathrm{SF}} \leq 0\right)$ represent that di-BODIPY meets the SF energy criterion in c-hex and the purple dots $\left(\Delta \mathrm{E}_{\mathrm{SF}}>0\right)$ represent that it doesn't in c-hex. (c) Time evolution of the ${ }^{2} \mathrm{HOMO}$ and ${ }^{2} \mathrm{LUMO}$ energies and the ${ }^{2} \mathrm{HOMO}-{ }^{2} \mathrm{LUMO}$ energy gaps of diBODIPY in $\mathrm{CHCl}_{3}$ and c-hex represented by 200 transient geometries extracted from their corresponding 2 ps AIMD simulation trajectories, respectively. The ${ }^{2} \mathrm{HOMO}(-6.55 \mathrm{eV})$ and ${ }^{2} \mathrm{LUMO}(-1.81 \mathrm{eV})$ energies and the ${ }^{2} \mathrm{HOMO}-{ }^{2} \mathrm{LUMO}$ energy gap $(4.74 \mathrm{eV})$ of the gaseous static diBODIPY are also shown for comparison (orange, olive and violet dash-dot lines).

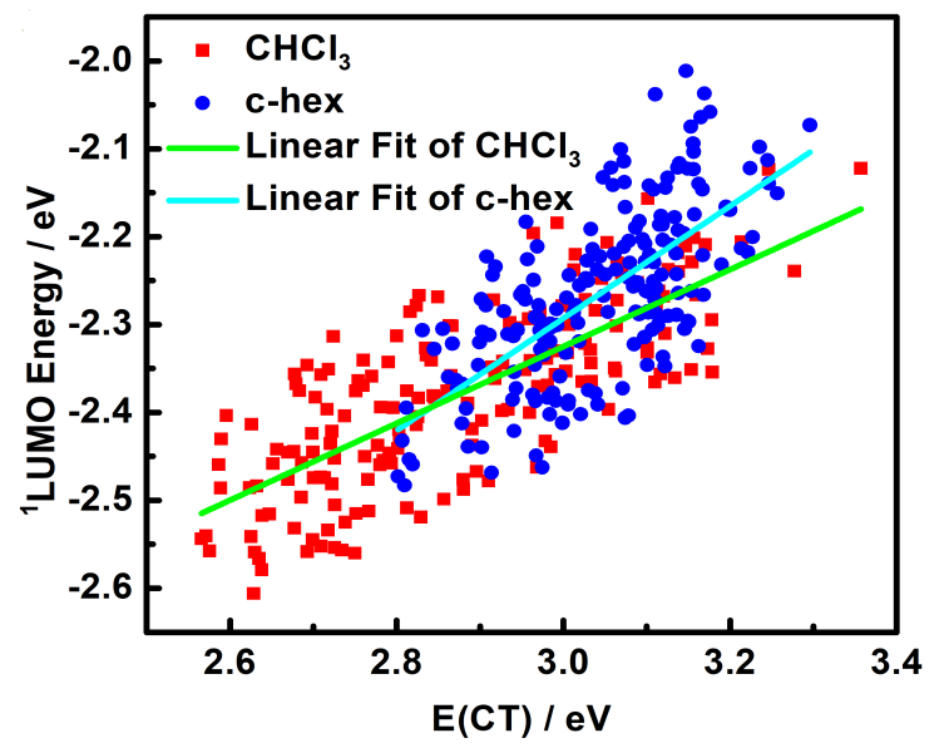

Figure S7. The relationship between the CT state energies and ${ }^{1}$ LUMO energies of di-BODIPY in $\mathrm{CHCl}_{3}$ (red dots) and cyclohexane (c-hex, blue dots) for 200 snapshot configurations, respectively. 


\section{Further Verification of the Proposed Dual-Switched Singlet Fission Channel for Di-BODIPY in Frozen DCM/toluene at $80 \mathrm{~K}$}

A recent work reported a different experimental observation about the photophysics of diBODIPY in a different experimental condition (in frozen DCM/toluene $(1: 1, \mathrm{v} / \mathrm{v})$ at $80 \mathrm{~K})(\mathrm{see}$ Ref 58. Kandrashkin, Y. E.; et al. Balance between triplet states in photoexcited orthogonal BODIPY dimers. J Phys Chem Lett. 2019, 10 (15), 4157-4163).

To clarify if our proposed channel can interpret that experimental phenomenon, AIMD simulation was performed on di-BODIPY in frozen DCM/toluene $(1: 1, \mathrm{v} / \mathrm{v})$ at $80 \mathrm{~K}$ for $2 \mathrm{ps}$ and transient configurations of di-BODIPY with six nearest neighbor solvent molecules were extracted (the sampling time stepsize $=10 \mathrm{fs}$ ) from the trajectory, and the results are shown in Figure S8. As illustrated in Figure S8, we find that the singlet and triplet vertical excitation energies vary with the time in frozen DCM/toluene $(1: 1, \mathrm{v} / \mathrm{v})$ at $80 \mathrm{~K}$. With the evolution of time, $\mathrm{E}\left(\mathrm{S}_{1}\right)$ varies in a range of $2.58-2.91 \mathrm{eV}$ with a small fluctuation $\left(\Delta \mathrm{E}\left(\mathrm{S}_{1}\right)=0.33 \mathrm{eV}\right)$, while $\mathrm{E}\left({ }^{2} \mathrm{~T}_{1}\right)$ varies in the between of $1.31-1.50 \mathrm{eV}$ and $\mathrm{E}\left({ }^{1} \mathrm{~T}_{1}\right)$ varies in the between of $1.42-1.67 \mathrm{eV}$, showing small fluctuations $\left(\Delta \mathrm{E}\left({ }^{2} \mathrm{~T}_{1}\right)=0.21 \mathrm{eV}\right.$ and $\left.\Delta \mathrm{E}\left({ }^{1} \mathrm{~T}_{1}\right)=0.25 \mathrm{eV}\right)$. More importantly, the probability of meeting SF energy criterion in frozen DCM/toluene at $80 \mathrm{~K}$ is $0 \%$. That is, $\Delta \mathrm{E}_{\mathrm{SF}}>0$ for all transient configurations, indicating that the SF channel is switched off when di-BODIPY in frozen DCM/toluene $(1: 1, \mathrm{v} / \mathrm{v})$ at $80 \mathrm{~K}$. When the SF thermodynamics requirement is not satisfied, the triplet excitons may be generated mainly through the ISC mechanism.

Clearly, using the proposed dual-switched SF channel, this AIMD simulation also reasonably interpret the experimental phenomenon about the photophysics of di-BODOPY in different solvent and condition (e.g. DCM/toluene (1:1, v/v) at $80 \mathrm{~K})$. 


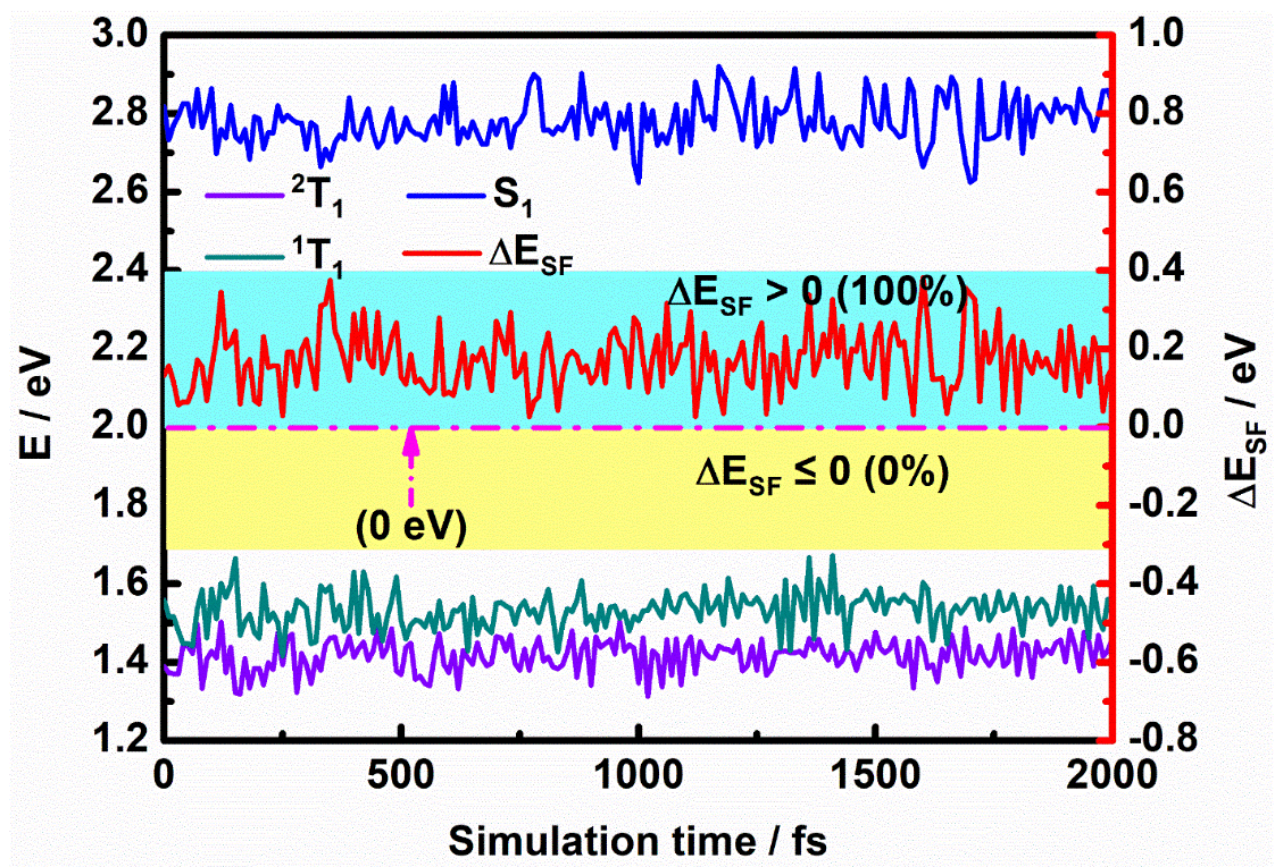

Figure S8. Time evolution of the smallest vertical excitation energies to the singlet excited state $\left(\mathrm{S}_{0} \rightarrow \mathrm{S}_{1}, \mathrm{E}\left(\mathrm{S}_{1}\right) /\right.$ blue curve $)$ and triplet excited state $\left(\mathrm{S}_{0} \rightarrow{ }^{1} \mathrm{~T}_{1}, \mathrm{E}\left({ }^{1} \mathrm{~T}_{1}\right) /\right.$ dark cyan curve and $\mathrm{S}_{0} \rightarrow{ }^{2} \mathrm{~T}_{1}$, $\mathrm{E}\left({ }^{2} \mathrm{~T}_{1}\right) /$ violet curve) and the SF driving force ( $\triangle \mathrm{ESF}$, red curve) of di-BODIPY in frozen DCM/toluene $(1: 1, \mathrm{v} / \mathrm{v})$ at $80 \mathrm{~K}$.

\section{Further Verification of the Proposed Dual-Switched Singlet Fission Channel Using Other System (a Covalent Pentacene Dimer in Benzonitrile)}

In order to verify the generality of the proposed dynamic dual-switched singlet fission channel, according to the reference [Ref 29. Zirzlmeier, J.; et al. Singlet fission in pentacene dimers. Proc. Natl. Acad. Sci. 2015, 112, 5325-5330], a covalent pentacene dimer (p-2, two pentacene are linked via a covalent phenylene spacer in para-arrangement) in benzonitrile solution was selected for further clarification which can undergo SF with high efficiency (see Figure S9). We constructed a cubic box with a side length of $21.415 \AA$ according to the ratio (1:50) of the covalent pentacene dimer $\boldsymbol{p}-2$ to benzonitrile solvent molecules, and AIMD simulation was performed on the system in benzonitrile for 1 ps and 100 transient configurations 
of the covalent pentacene dimer $\boldsymbol{p}$ - 2 with six nearest neighbor solvent molecules were extracted (the time stepsize $=10 \mathrm{fs}$ ) from the trajectory. The calculated results are given in Figure S9, Table S20 and Figure S10. As illustrated in Figure S10(a), we find that the singlet and triplet excitation energies vary with the time in benzonitrile. More importantly, the probability of meeting SF energy criterion $\left(\Delta \mathrm{E}_{\mathrm{SF}} \leq 0\right)$ is $82 \%$, indicating that the SF channel is switched on with a very high probability for such a covalent pentacene dimer in benzonitrile and thus is thermodynamically favorable. We also examined $\Delta \mathrm{E}_{\mathrm{CT}-\mathrm{S} 1}$ of the 100 transient configurations of p-2 in benzonitrile and monitored their time evolution dynamics (Figure S10(a), red curve). The CT state energy oscillatorily varies with the time in benzonitrile solvent (blue curve). Interestingly, $\Delta \mathrm{E}_{\mathrm{CT}-\mathrm{S} 1} \leq 0$ exhibits a probability of ca. $75 \%$ in a 1 ps time window for $\boldsymbol{p}-\mathbf{2}$ in benzonitrile. For all 100 transient configurations appearing in a 1 ps AIMD simulation trajectory, the probability distributions for if meeting the two energy criteria are classified into 4 cases (Figure S10(b)). The probabilities meeting the thermodynamic criterion $\left(\Delta \mathrm{E}_{\mathrm{SF}} \leq 0\right.$, Switch $1 \mathrm{ON})$ and the kinetic criterion $\left(\Delta \mathrm{E}_{\mathrm{CT}-\mathrm{S} 1} \leq 0\right.$, Switch $\left.2 \mathrm{ON}\right)$ simultaneously is $61 \%$. The probability not meeting simultaneously the two criteria $\left(\Delta \mathrm{E}_{\mathrm{SF}}>0, \Delta \mathrm{E}_{\mathrm{CT}-\mathrm{S} 1}>0\right)$ is only $5 \%$ (both Switches OFF). These distributions indicate that not all transient perturbations can yield effective configurations for SF in such a covalent pentacene dimer in benzonitrile but the effective configurations are up to $61 \%$ in benzonitrile, a very high probability of SF.

Clearly, this additional AIMD simulation on different system also confirms the generality of the proposed dual-switched singlet fission channel in solution. 


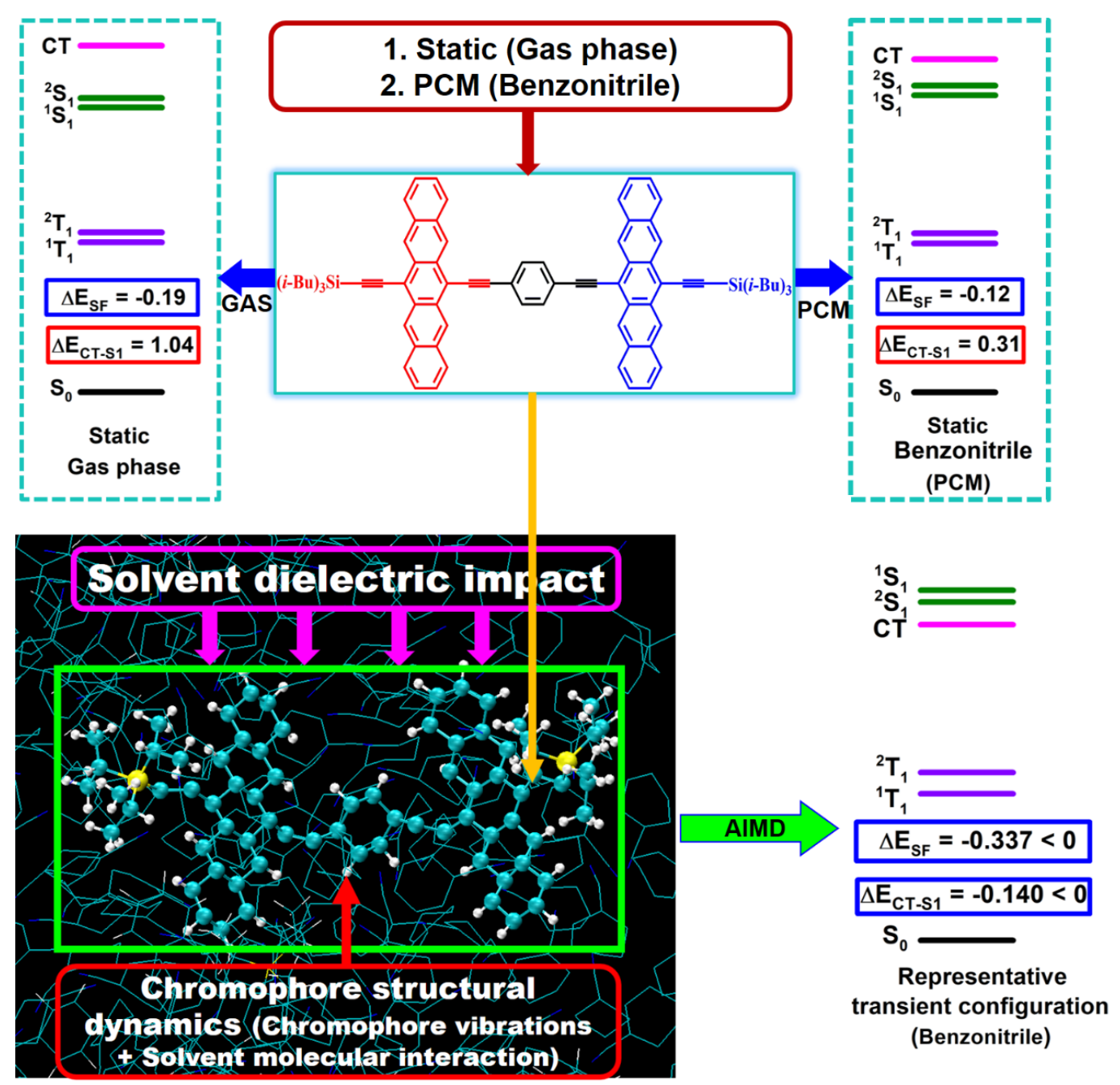

Figure S9. Chromophore Structure and Energetics Properties. A schematic structure of a covalent pentacene dimer linked via a phenylene spacer in para-arrangement $(\boldsymbol{p}-2)$ and the vertically excited state energies of the ground state $\boldsymbol{p}-\mathbf{2}$ in the gas phase (upper left panel). The ground state $\boldsymbol{p}-\mathbf{2}$ and the corresponding excited state energies in the PCM-based benzonitrile solvent (upper right panel). A transient configuration extracted from a 1 ps AIMD simulation trajectory of $\boldsymbol{p}-\mathbf{2}$ in benzonitrile (lower left panel) and the excited state energies of a representative transient configuration of $\boldsymbol{p}-\mathbf{2}$ in benzonitrile (lower right panel). $\Delta \mathrm{E}_{\mathrm{SF}} \leq 0$ and $\Delta \mathrm{E}_{\mathrm{CT} \text {-S1 }} \leq 0$ denote that $p-2$ in benzonitrile meets both the SF thermodynamic and kinetic conditions, while $\Delta \mathrm{E}_{\mathrm{SF}}>0$ and $\Delta \mathrm{E}_{\mathrm{CT}-\mathrm{S} 1}>0$ denote both not meeting. 


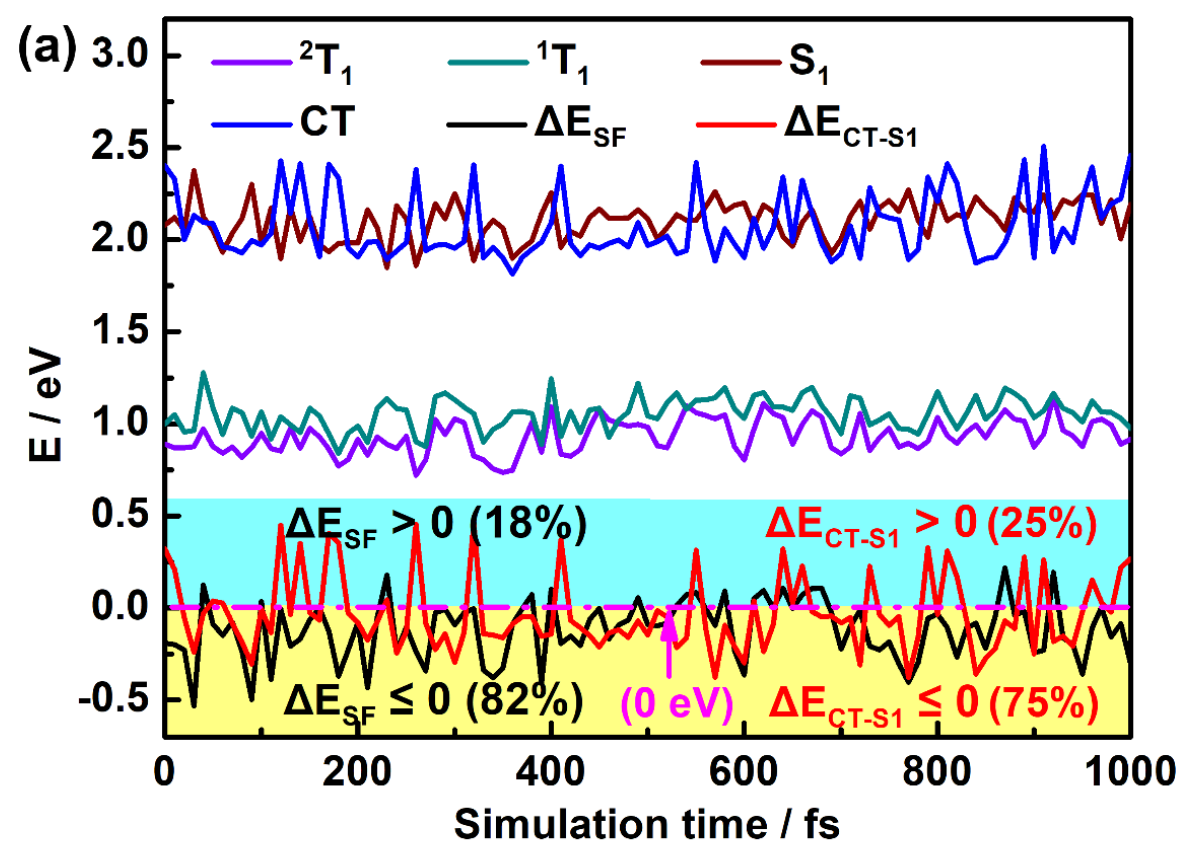

(b)

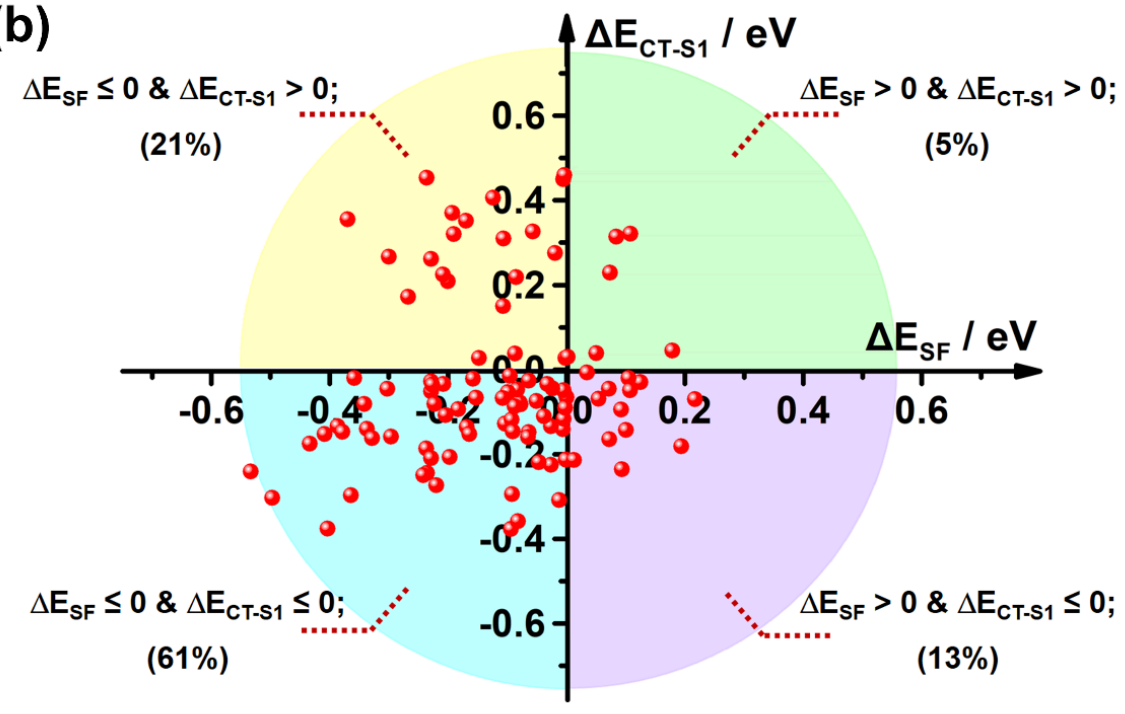

Figure S10. (a) Time evolution of the smallest vertical excitation energies to the first singlet excited state $\left(\mathrm{S}_{0} \rightarrow \mathrm{S}_{1}, \mathrm{E}\left(\mathrm{S}_{1}\right) /\right.$ wine curve $)$ and first triplet excited state $\left(\mathrm{S}_{0} \rightarrow{ }^{1} \mathrm{~T}_{1}, \mathrm{E}\left({ }^{1} \mathrm{~T}_{1}\right) /\right.$ dark cyan curve and $\mathrm{S}_{0} \rightarrow{ }^{2} \mathrm{~T}_{1}, \mathrm{E}\left({ }^{2} \mathrm{~T}_{1}\right) /$ violet curve) and $\mathrm{CT}$ state (blue curve), the SF driving force $\left(\Delta \mathrm{E}_{\mathrm{SF}}\right.$, black curve $)$ and the kinetic barriers ( $\Delta \mathrm{E}_{\mathrm{CT}-\mathrm{S} 1}$, red curve) of the covalent pentacene dimer $\boldsymbol{p}-\mathbf{2}$ in benzonitrile. (b) The correlation distribution between the thermodynamic driving force $\left(\Delta \mathrm{E}_{S F}\right)$ and kinetic barrier $\left(\Delta \mathrm{E}_{\mathrm{CT}-\mathrm{S} 1}\right)$ and the probability statistics according to the $\Delta \mathrm{E}_{\mathrm{SF}}$ and $\Delta \mathrm{E}_{\mathrm{CT}-\mathrm{S} 1}$ criteria for SF in the covalent pentacene dimer $\boldsymbol{p}-\mathbf{2}$ in benzonitrile for all 100 transient configurations. 
Table S20. Excited State Energies of the Optimized Ground State Structure of the Covalent Pentacene Dimer $\boldsymbol{p}-\mathbf{2}$ in the Gas Phase and Benzonitrile

$$
\mathrm{S}_{1}(\mathrm{eV}) \quad{ }^{1} \mathrm{~T}_{1}(\mathrm{eV}) \quad{ }^{2} \mathrm{~T}_{1}(\mathrm{eV}) \quad \mathrm{CT}(\mathrm{eV}) \quad \Delta \mathrm{E}_{\mathrm{SF}}(\mathrm{eV}) \quad \Delta \mathrm{E}_{\mathrm{CT}-\mathrm{S} 1}(\mathrm{eV})
$$

$\begin{array}{lllllll}\text { Gas phase } & 2.08 & 0.89 & 1.00 & 3.12 & -0.19 & 1.04\end{array}$

$\begin{array}{lllllll}\text { Benzonitrile } & 2.12 & 0.94 & 1.06 & 2.43 & -0.12 & 0.31\end{array}$

Note: $\Delta \mathrm{E}_{\mathrm{SF}}=\mathrm{E}\left({ }^{2} \mathrm{~T}_{1}\right)+\mathrm{E}\left({ }^{1} \mathrm{~T}_{1}\right)-\mathrm{E}\left(\mathrm{S}_{1}\right) ; \Delta \mathrm{E}_{\mathrm{CT}-\mathrm{S} 1}=\mathrm{E}(\mathrm{CT})-\mathrm{E}\left(\mathrm{S}_{1}\right) ;$ all are calculated at the M062X/cc-pVDZ level.

\section{Additional AIMD Simulation for di-BODIPY in $\mathrm{CHCl}_{3}$ (the Other Trajectory)}

In order to verify the representativeness of the solvent effect in the system and the correctness of the probability statistics, we set a new initial configuration of di-BODIPY in $\mathrm{CHCl}_{3}$ solvent, and then AIMD simulation was carried out for further equilibration for $5 \mathrm{ps}$. We extracted di-BODIPY with six nearest neighbor solvent molecules per $10 \mathrm{fs}$ at the time window of 4500-5000 fs from this trajectory and calculated $\mathrm{E}\left(\mathrm{S}_{1}\right), \mathrm{E}\left({ }^{1} \mathrm{~T}_{1}\right), \mathrm{E}\left({ }^{2} \mathrm{~T}_{1}\right)$ and $\mathrm{E}(\mathrm{CT})$ of each transient geometry at the TD-M062X/cc-pVDZ level for each case, results are shown in Figure S11. As illustrated in Figure S11, the statistical results of the probability distribution of the two trajectories are approximately the same, which verifies the accuracy of our results. 


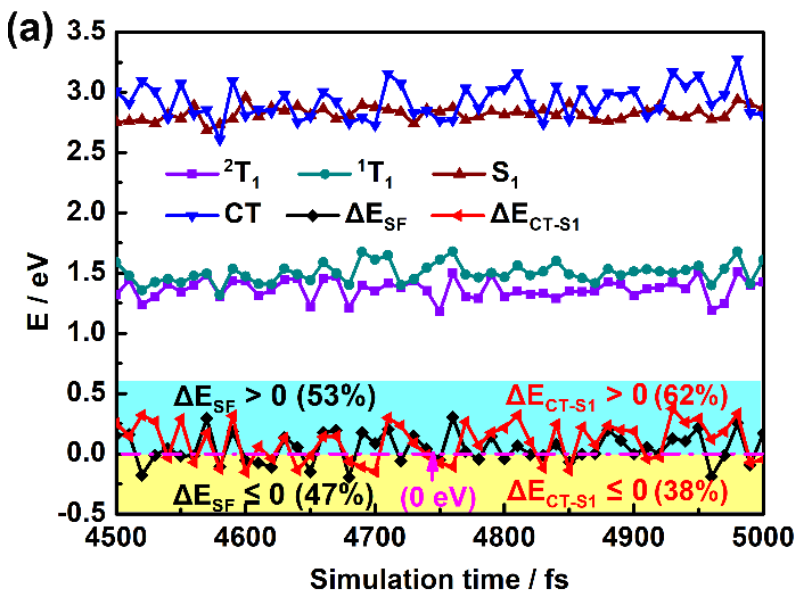

(b)

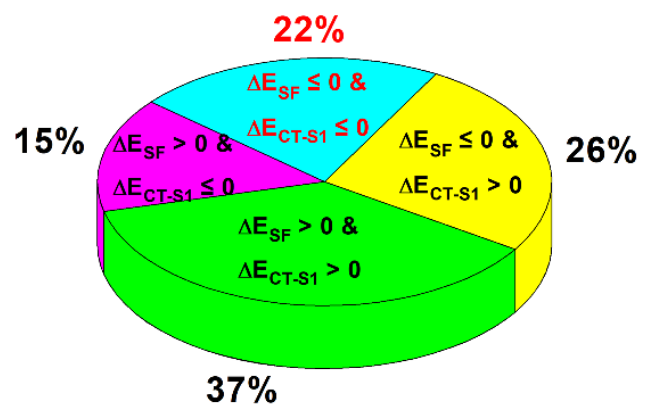

Figure S11. (a) Time evolution of the smallest vertical excitation energies to the singlet excited state $\left(\mathrm{S}_{0} \rightarrow \mathrm{S}_{1}, \mathrm{E}\left(\mathrm{S}_{1}\right) /\right.$ wine curve $)$ and triplet excited state $\left(\mathrm{S}_{0} \rightarrow{ }^{1} \mathrm{~T}_{1}, \mathrm{E}\left({ }^{1} \mathrm{~T}_{1}\right) /\right.$ dark cyan curve and $\mathrm{S}_{0} \rightarrow{ }^{2} \mathrm{~T}_{1}, \mathrm{E}\left({ }^{2} \mathrm{~T}_{1}\right) /$ violet curve) and CT state (blue curve) the SF driving force ( $\Delta \mathrm{E}_{\mathrm{SF}}$, black curve) and the kinetic barriers ( $\triangle \mathrm{E}_{\mathrm{CT}-\mathrm{S} 1}$, red curve) of di-BODIPY in $\mathrm{CHCl}_{3}$ at the time window of 4500 5000 fs for another dynamics trajectory. (b) The probability statistics according to the thermodynamic $\left(\Delta \mathrm{E}_{\mathrm{SF}}\right)$ and kinetic $\left(\Delta \mathrm{E}_{\mathrm{CT}-\mathrm{S} 1}\right)$ criteria for 50 transient geometries in $\mathrm{CHCl}_{3}$ in which the probability of meeting both the energy requirements $\left(\Delta \mathrm{E}_{\mathrm{SF}} \leq 0\right.$ and $\left.\Delta \mathrm{E}_{\mathrm{CT}-\mathrm{S} 1} \leq 0\right)$ is $22 \%$ (red area).

\section{Time Evolution of the Dihedral Angles and Bond Lengths}

Dihedral Angle Variations. Undoubtedly, all of the above-observed phenomena including variations of the excitation energies of singlet, triplet and CT states, and oscillation of the SF driving force must be associated with structural changes of di-BODIPY in solvents along the evolution time. To further clarify how the structural fluctuations induced by solvents and solvent polarity affect the SF mechanism, we examine the geometrical parameters of the extracted snapshot configurations. We find that the main structural distortions originate from the variations of the dihedral angle between two BODIPY fragments (Figure S12). From the above analyses, thermodynamics condition and the kinetics condition jointly serve a double switch and cooperatively control the SF channel of di-BODIPY with the ON state in polar solvents. Thus, the analyses of singlet pre-fission must take these two conditions into account. But, it is very difficult to determine how the dihedral angle governs the above observed irregular variations of pre-SF energetic quantities (Figure S13). 

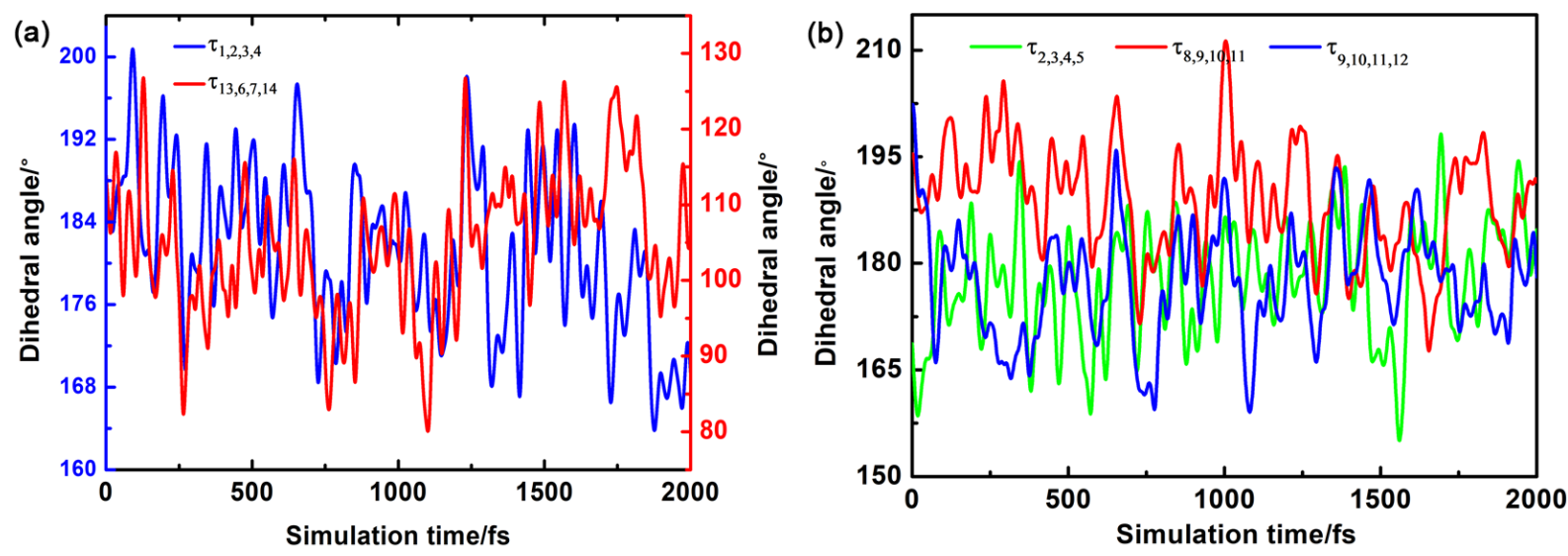

Figure S12. (a) Time evolution of the dihedral angle $\tau_{1,2,3,4}$ (blue line) and dihedral angle $\tau_{13,6,7,14}$ (red line) of di-BODIPY in $\mathrm{CHCl}_{3}$.

(b) Time evolution of the dihedral angle $\tau_{2,3,4,5}$ (green line), dihedral angle $\tau_{8,9,10,11}$ (red line) and dihedral angle $\tau_{9,10,11,12}$ (blue line) of di-BODIPY in $\mathrm{CHCl}_{3}$.
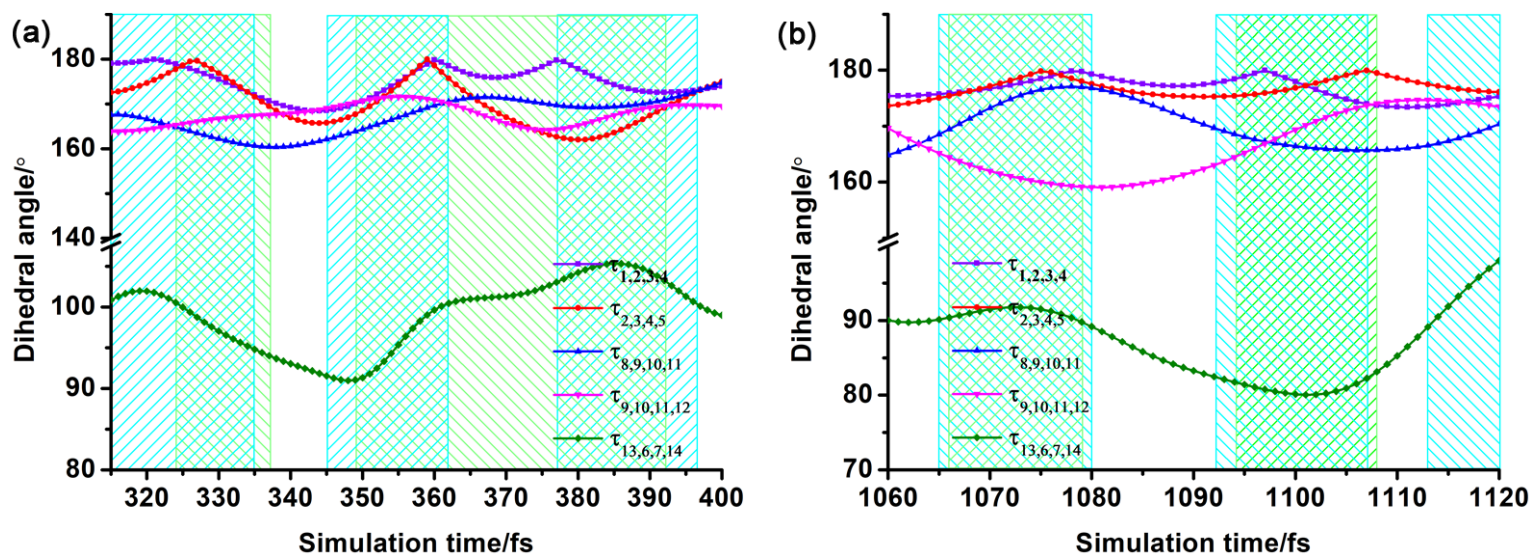

Figure S13. Variations of five dihedral angles $\left(^{\circ}\right)$ in time evolution of the snapshot configurations extracted every 1 fs in a time window of 315-400 fs (a) and 1060-1120 fs (b) from the AIMD simulation trajectory with respect to those of di-BODIPY in $\mathrm{CHCl}_{3}$. The sparse green-line filled areas denote the time ranges in which di-BODIPY meets the SF energy criterion. The sparse cyan-line filled areas denote the time ranges in which di-BODIPY meets the kinetics condition $(\mathrm{E}(\mathrm{CT}) \leq$ $\left.\mathrm{E}\left(\mathrm{S}_{1}\right)\right)$. Overlapping parts denote the time ranges in which di-BODIPY undergoes SF.

Bond Length Variations. We find that the bond length varies periodically and the variation periods are short and irregular. Therefore, we checked nine typical bond lengths of some 
representative snapshot configurations of di-BODIPY in $\mathrm{CHCl}_{3}$ (Figure $\mathbf{S 1 4}$ to S15). As illustrated in Figure S15, the dense cyan-line filled areas denote that in those time ranges the snapshot configurations of di-BODIPY meets both the conditions, $\Delta \mathrm{E}_{\mathrm{SF}} \leq 0$ and $\Delta \mathrm{E}_{\mathrm{CT}-\mathrm{S} 1} \leq 0$, that is, diBODIPY can undergo SF in those time periods. Figure S15 also reveals that di-BODIPY undergoes SF only when the C6-C7 bond length is shorter and the two bond lengths connected to B atoms are quite different (i.e. N2-B3 > B3-N4 or B10-N11 > N9-B10). This phenomenon can be understood by the fact that the $\mathrm{B}$ atom is prone to swing left or right due to the influence of $\mathrm{CHCl}_{3}$, which makes resonant conversion of the coordination bond and covalent bond of di-BODIPY $(\mathrm{N}: \rightarrow \mathrm{B}-\mathrm{N} \leftrightarrow \mathrm{N}-\mathrm{B} \leftarrow: \mathrm{N})$, favoring $\mathrm{SF}$.

\section{In short, the main geometrical parameter changes that are favorable to SF (including the main} roles) can be outlined in Figure S16.
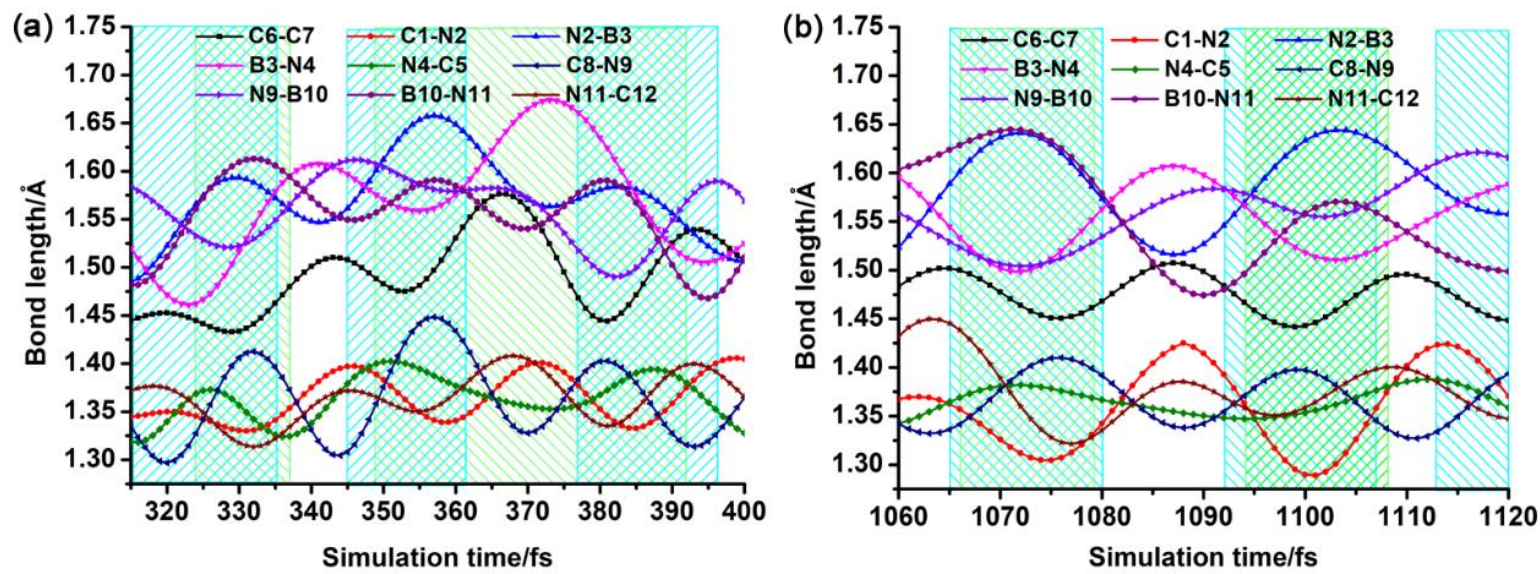

Figure S14. Time evolution of lengths of nine bonds $(\AA)$ of the snapshot configurations extracted every $1 \mathrm{fs}$ in a time window of 315-400 fs (a) and 1060-1120 fs (b) from the AIMD simulation trajectory with respect to those of di-BODIPY in $\mathrm{CHCl}_{3}$. The sparse green-line filled areas denote the time ranges in which the di-BODIPY meets the SF energy criterion. The sparse cyan-line filled areas denote the time ranges in which di-BODIPY meets the kinetics condition $\left(E(C T) \leq E\left(S_{1}\right)\right)$. Overlapping parts denote the time ranges in which di-BODIPY undergoes SF. 

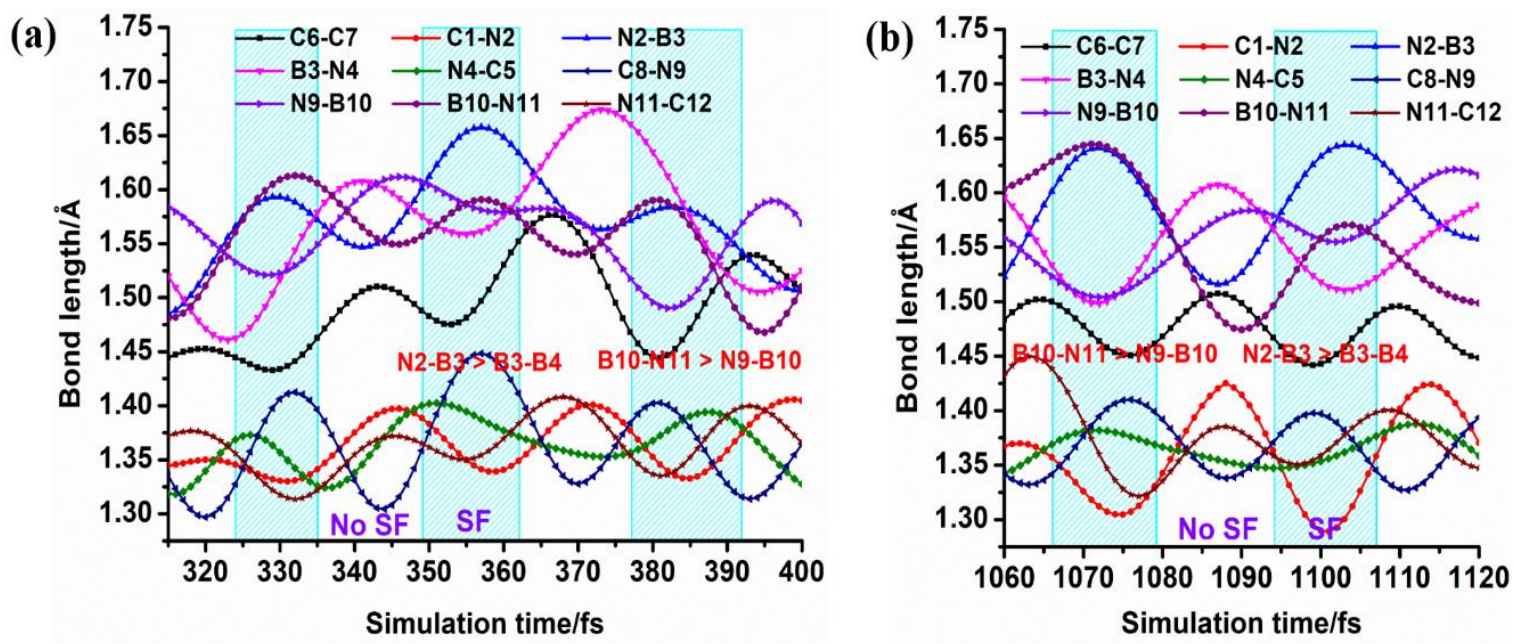

Figure S15. Time evolution of lengths of nine bonds $(\AA)$ of the snapshot configurations extracted every 1 fs in two time windows of 315-400 fs (a) and 1060-1120 fs (b) from a 2 ps AIMD simulation trajectory for di-BODIPY in $\mathrm{CHCl}_{3}$. The dense cyan-line filled areas denote the time ranges in which di-BODIPY undergoes SF.

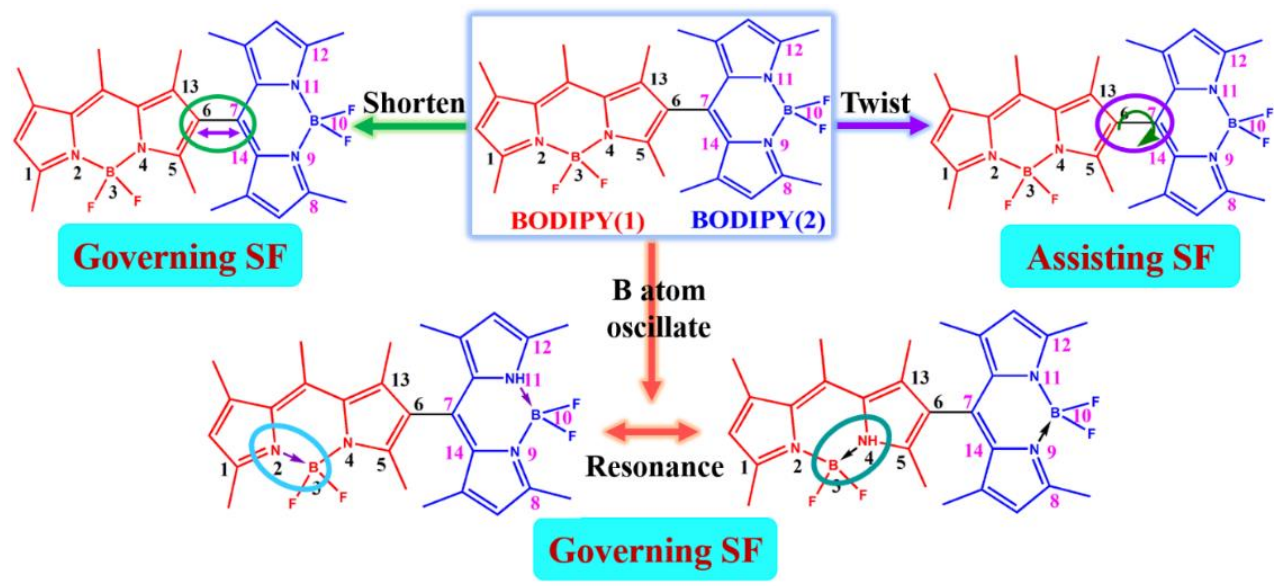

Figure S16. Main structural changes for governing SF. These structural changes include approach of two BODIPY (shortening of the C6-C7 bond) and/or B atom oscillation between its linked two $\mathrm{N}$ atoms in each BODIPY (resonant conversion between $\mathrm{N}-\mathrm{B} \leftarrow: \mathrm{N} \leftrightarrow \mathrm{N}: \rightarrow \mathrm{B}-\mathrm{N}$ ) but the resonant effect in BODIPY(1) is smaller than that in BODIPY(2), and in the latter the N2: $\rightarrow$ B3$\mathrm{N} 4$ resonant structure (featuring a shortened B3-N4 bond and elongated N2-B3 bond plays a dominant role in promoting SF. The dihedral twisting of two BODIPY plays an assisting role in governing SF due to small variation magnitudes. 


\section{Comparison of the CT State Energies and Their Frontier Molecular Orbitals of di-BODIPY in $\mathrm{CHCl}_{3}$ and cyclohexane Solvent Using Different Functionals}

To verify the correctness of the CT state energy at the M062X functional level, we extracted 10 transient configurations of di-BODIPY in $\mathrm{CHCl}_{3}$ and cyclohexane from their AIMD simulation trajectories, respectively, and then calculated their CT state energies using the M062X, B3LYP and CAM-B3LYP functionals and the cc-pVDZ basis set. Relevant results are shown in Figure S17 to S18. Although the CT state energies are slightly different for these functionals and a different basis set, the trend of CT state energies is the same for them. Most importantly, there is no change about $\Delta \mathrm{E}_{\mathrm{CT}-\mathrm{S} 1}>0$ or $\Delta \mathrm{E}_{\mathrm{CT}-\mathrm{S} 1} \leq 0$ of transient configurations for these different functionals. These indicate that the M062X/cc-pVDZ method is reliable in studying the CT state energetics for the present system.

Comparison of the calculated transitions between the B3LYP/cc-pVDZ and CAMB3LYP/cc-pVDZ methods are given in Table $\mathbf{S 2 1}$ to $\mathbf{S 2 4}$.

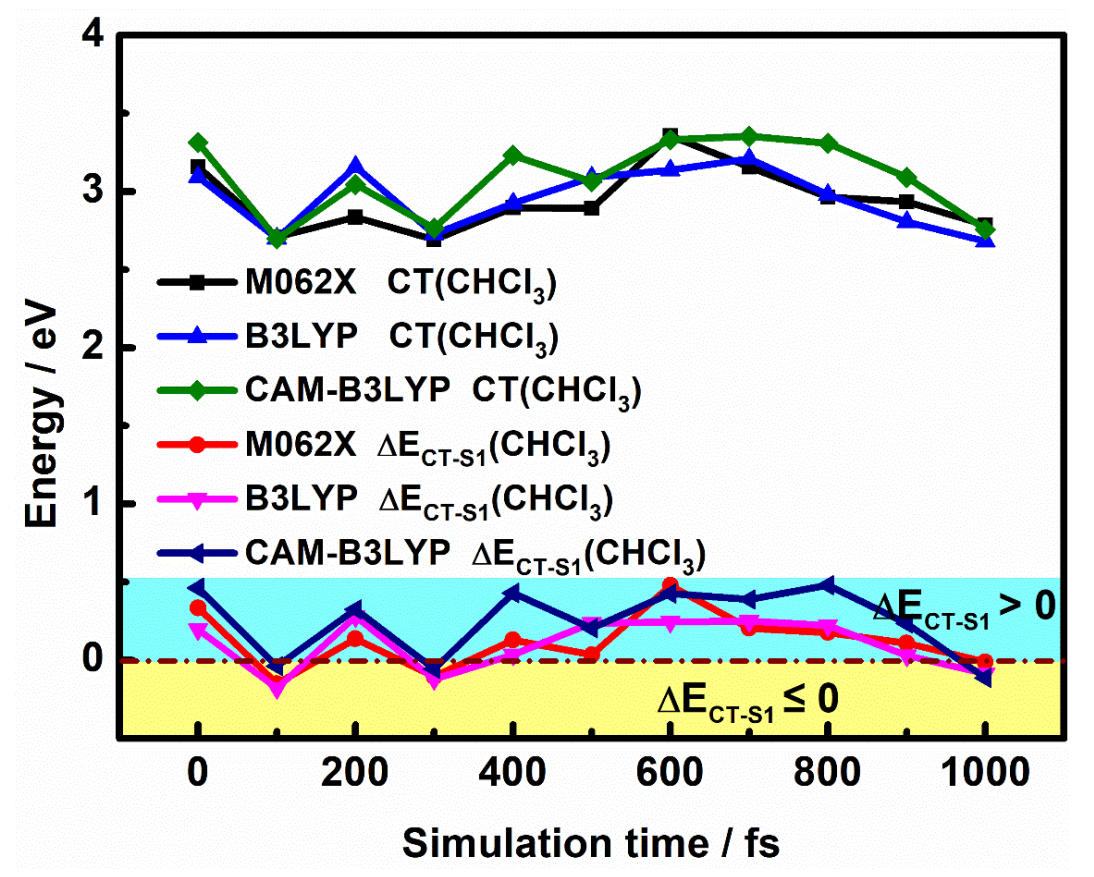

Figure S17. The CT state energies $\left(\mathrm{E}(\mathrm{CT})\right.$ ) of di-BODIPY in $\mathrm{CHCl}_{3}$ using the $\mathrm{M} 062 \mathrm{X}$ (black line), B3LYP (blue line) and CAM-B3LYP (olive line) functionals and the cc-pVDZ basis set. $\Delta \mathrm{E}_{\mathrm{CT}-\mathrm{S} 1}$ for the conversion of $\mathrm{S}_{1}$ to $\mathrm{CT}$ in $\mathrm{CHCl}_{3}$ using the M062X (red line), B3LYP (magenta line) and CAM-B3LYP (navy line) functionals are also displayed. 


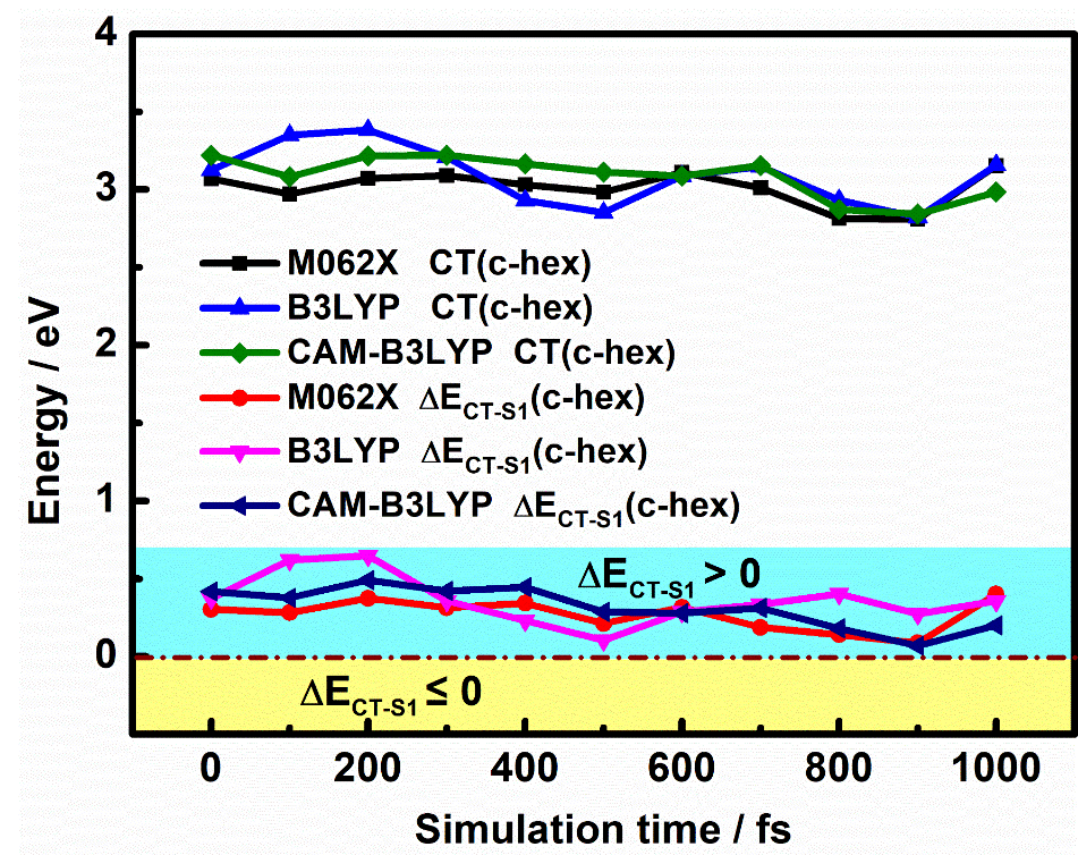

Figure S18. The CT state energies $(\mathrm{E}(\mathrm{CT}))$ of di-BODIPY in cyclohexane (c-hex) using the M062X (black line), B3LYP (blue line) and CAM-B3LYP (olive line) functionals and the ccpVDZ basis set. $\Delta \mathrm{E}_{\mathrm{CT}-\mathrm{S} 1}$ for the conversion of $\mathrm{S}_{1}$ to $\mathrm{CT}$ in c-hex using the M062X (red line), B3LYP (magenta line) and CAM-B3LYP (navy line) functionals are also displayed.

Table S21. Electron Transitions of Excited States and Their Corresponding Frontier Molecular Orbitals of Selected Transient Configurations for di-BODIPY in $\mathrm{CHCl}_{3}$ at the B3LYP/cc-pVDZ Level

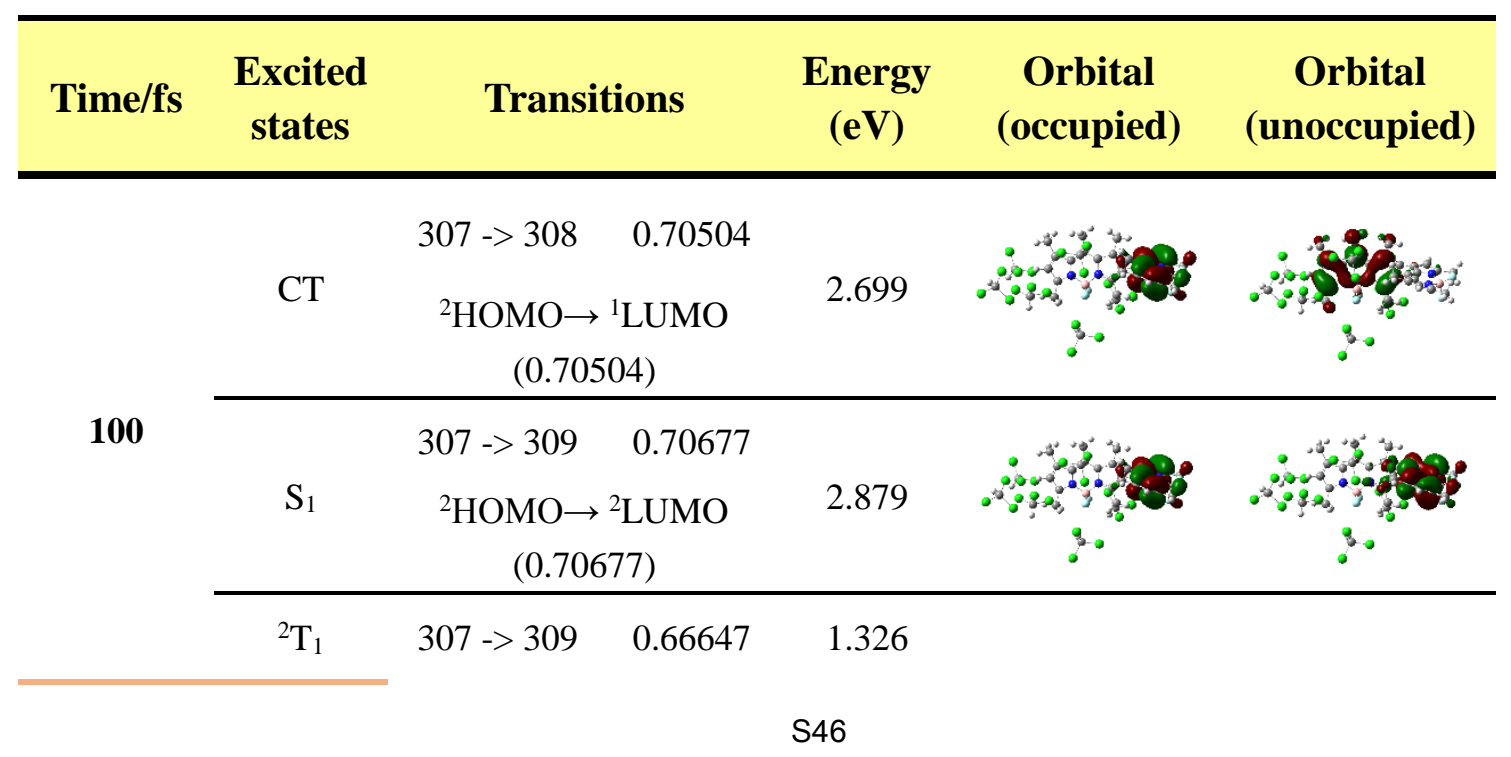




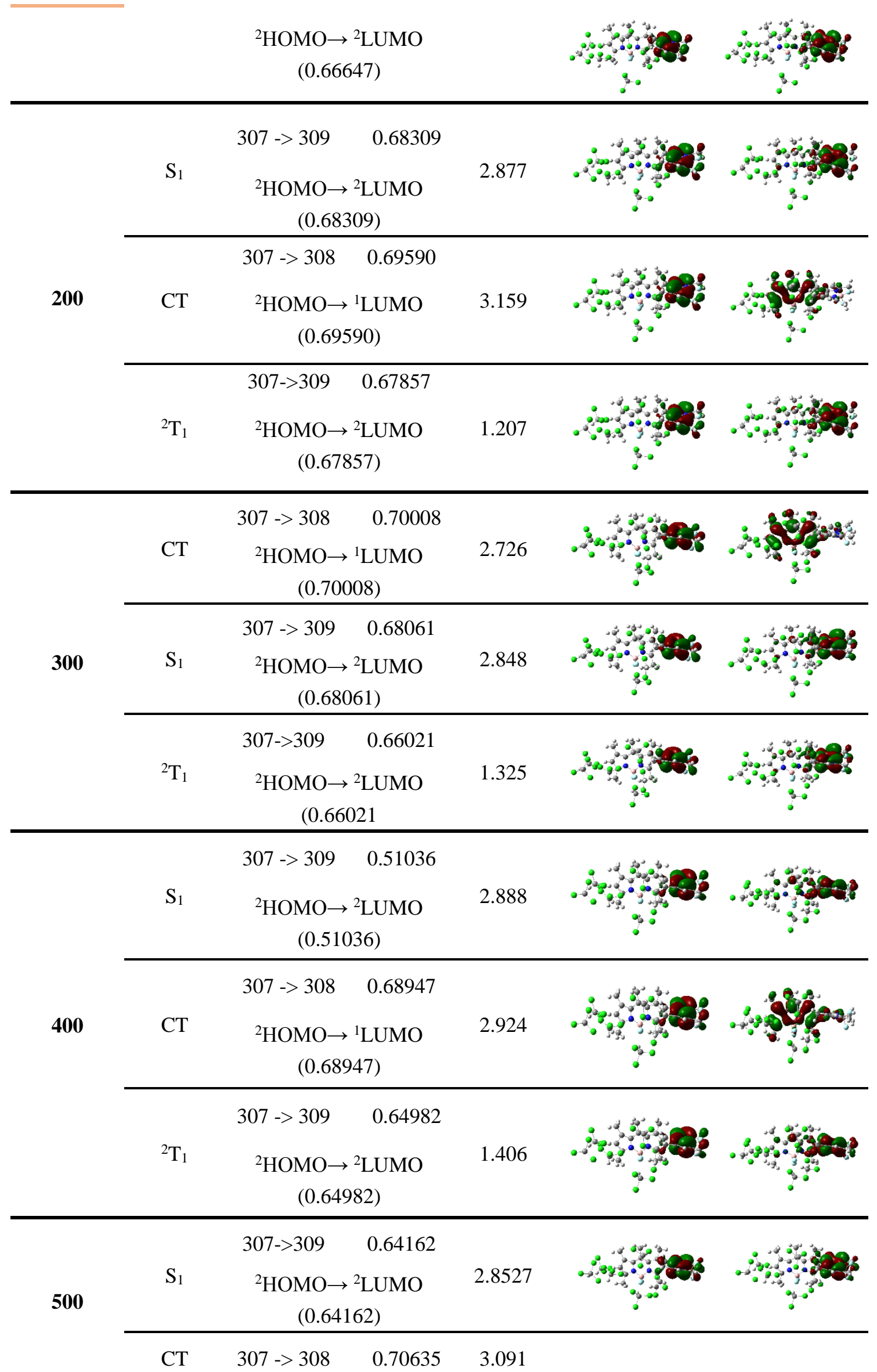


${ }^{2} \mathrm{HOMO} \rightarrow{ }^{1} \mathrm{LUMO}$

$(0.70635)$

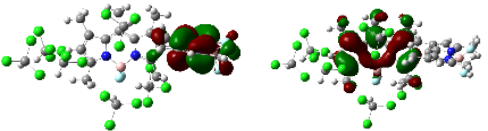

$307->309 \quad 0.71513$

${ }^{2} \mathrm{~T}_{1} \quad{ }^{2} \mathrm{HOMO} \rightarrow{ }^{2} \mathrm{LUMO}$

1.314

$(0.71513)$

Table S22. Electron Transitions of Excited States and Their Corresponding Frontier Molecular Orbitals of Selected Transient Configurations for di-BODIPY in $\mathrm{CHCl}_{3}$ at the CAM-B3LYP/ccpVDZ Level

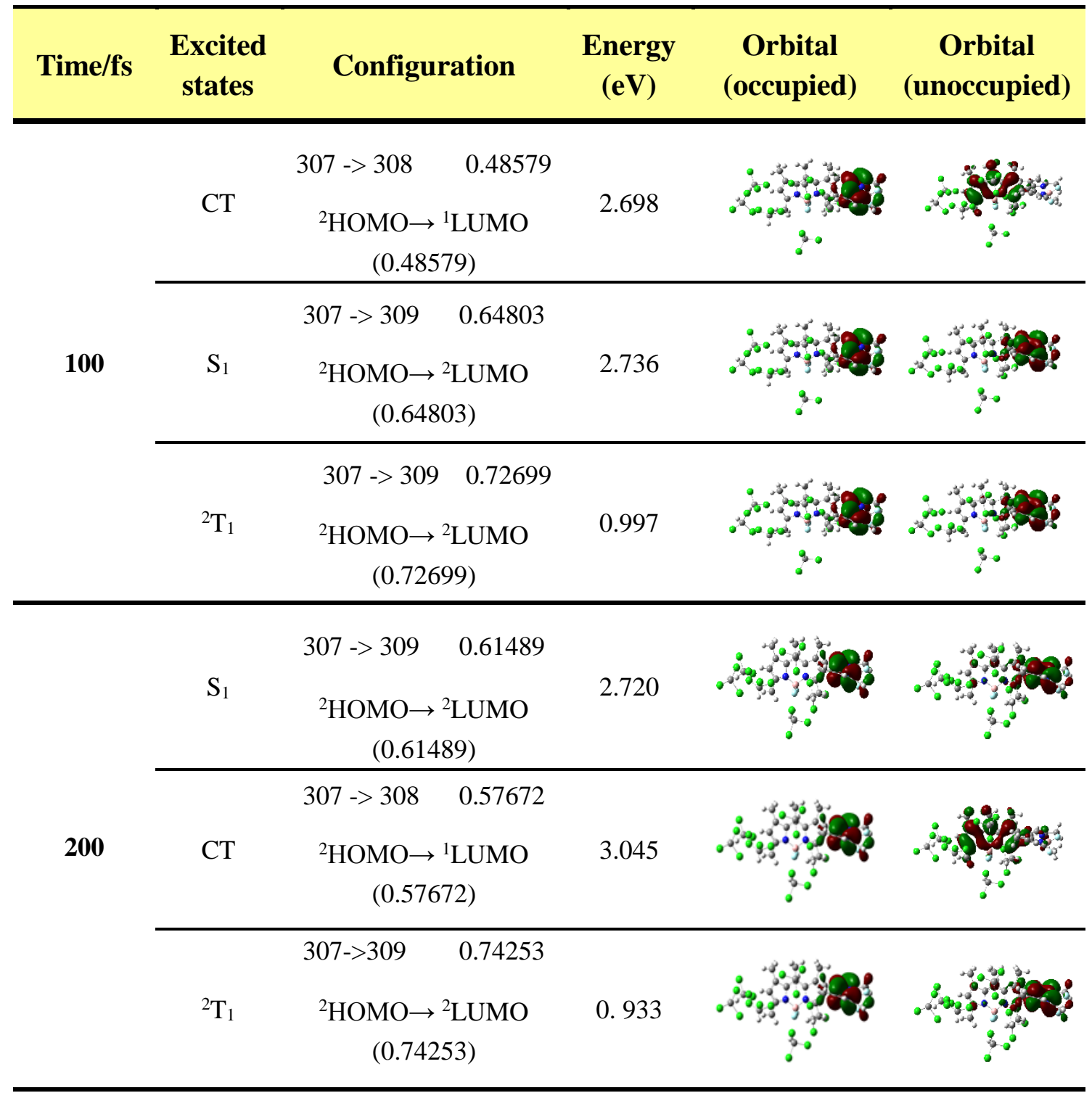




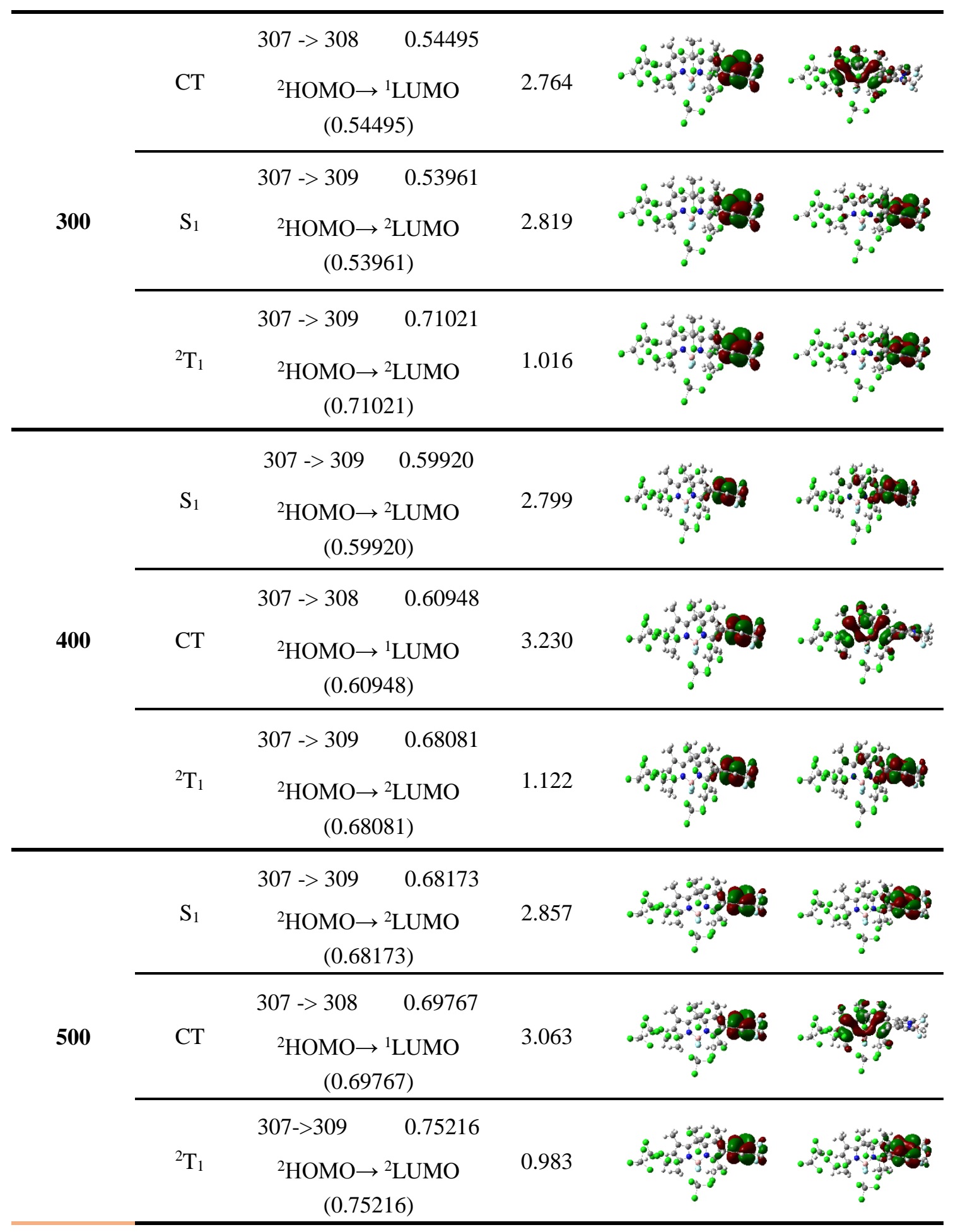


Table S23. Electron Transitions of Excited States and Their Corresponding Frontier Molecular Orbitals of Selected Transient Configurations for di-BODIPY in cyclohexane at the B3LYP/ccpVDZ Level

\begin{tabular}{|c|c|c|c|c|c|}
\hline Time/fs & $\begin{array}{c}\text { Excited } \\
\text { states }\end{array}$ & Configuration & $\begin{array}{c}\text { Energy } \\
(\mathrm{eV})\end{array}$ & $\begin{array}{c}\text { Orbital } \\
\text { (occupied) }\end{array}$ & $\begin{array}{c}\text { Orbital } \\
\text { (unoccupied) }\end{array}$ \\
\hline \multirow{3}{*}{100} & \multicolumn{2}{|r|}{$\begin{array}{c}276->278 \quad 0.68317 \\
{ }^{1} \mathrm{HOMO} \rightarrow{ }^{1} \mathrm{LUMO} \\
(0.68317) \\
\end{array}$} & 2.730 & \multirow{2}{*}{ 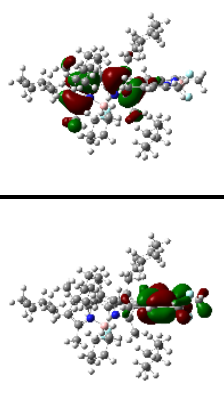 } & \\
\hline & $\mathrm{CT}$ & $\begin{array}{c}277->278 \quad 0.70276 \\
{ }^{2} \mathrm{HOMO} \rightarrow{ }^{1} \mathrm{LUMO} \\
(0.50801)\end{array}$ & 3.351 & & \\
\hline & ${ }^{2} \mathrm{~T}_{1}$ & $\begin{array}{c}277->279 \quad 0.70817 \\
{ }^{2} \mathrm{HOMO} \rightarrow{ }^{2} \mathrm{LUMO} \\
(0.70817)\end{array}$ & 1.287 & & \\
\hline \multirow{3}{*}{200} & $\mathrm{~S}_{1}$ & $\begin{array}{c}276->278 \quad 0.61563 \\
{ }^{1} \mathrm{HOMO} \rightarrow{ }^{1} \mathrm{LUMO} \\
(0.61563) \\
\end{array}$ & 2.736 & \multirow{2}{*}{ 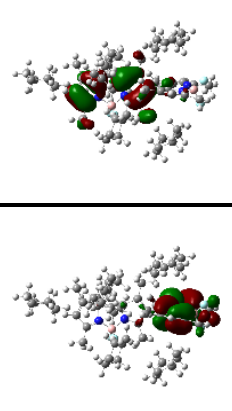 } & 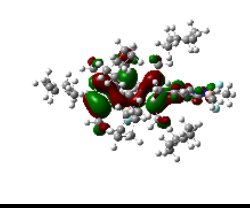 \\
\hline & $\mathrm{CT}$ & $\begin{array}{c}277->278 \quad 0.68436 \\
{ }^{2} \mathrm{HOMO} \rightarrow{ }^{1} \mathrm{LUMO} \\
(0.68436)\end{array}$ & 3.383 & & \\
\hline & ${ }^{2} \mathrm{~T}_{1}$ & $\begin{array}{c}277->279 \quad 0.65905 \\
{ }^{2} \mathrm{HOMO} \rightarrow{ }^{2} \mathrm{LUMO} \\
(0.65905)\end{array}$ & 1.339 & & \\
\hline \multirow{3}{*}{300} & $\mathrm{~S}_{1}$ & $\begin{array}{c}277->279 \quad 0.59460 \\
{ }^{2} \mathrm{HOMO} \rightarrow{ }^{2} \mathrm{LUMO} \\
(0.59460)\end{array}$ & 2.852 & 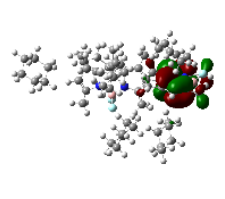 & 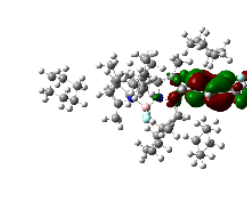 \\
\hline & $\mathrm{CT}$ & $\begin{array}{c}277->278 \quad 0.69069 \\
{ }^{2} \mathrm{HOMO} \rightarrow{ }^{1} \mathrm{LUMO} \\
(0.69069)\end{array}$ & 3.208 & \multirow[t]{2}{*}{ 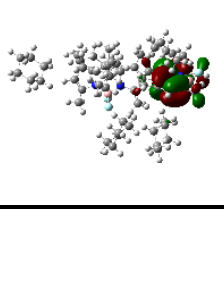 } & \multirow[t]{2}{*}{ 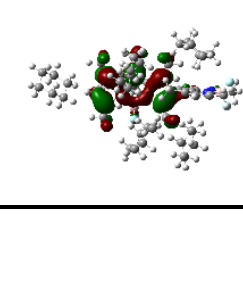 } \\
\hline & ${ }^{2} \mathrm{~T}_{1}$ & 277 -> 279 & 1.402 & & \\
\hline
\end{tabular}




$$
\begin{gathered}
{ }^{2} \mathrm{HOMO} \rightarrow{ }^{2} \mathrm{LUMO} \\
(0.64170)
\end{gathered}
$$

Table S24. Electron Transitions of Excited States and Their Corresponding Frontier Molecular Orbitals of Selected Transient Configurations for di-BODIPY in cyclohexane at the CAM-

\begin{tabular}{|c|c|c|c|c|c|}
\hline Time/fs & $\begin{array}{c}\text { Excited } \\
\text { states }\end{array}$ & Configuration & $\begin{array}{c}\text { Energy } \\
(\mathrm{eV})\end{array}$ & $\begin{array}{c}\text { Orbital } \\
\text { (occupied) }\end{array}$ & $\begin{array}{c}\text { Orbital } \\
\text { (unoccupied) }\end{array}$ \\
\hline \multirow{3}{*}{100} & $\mathrm{~S}_{1}$ & $\begin{array}{c}276->278 \quad 0.68406 \\
{ }^{1} \mathrm{HOMO} \rightarrow{ }^{1} \mathrm{LUMO} \\
(0.68406)\end{array}$ & 2.705 & $\log ^{2}$ & 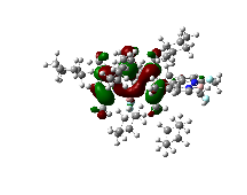 \\
\hline & $\mathrm{CT}$ & $\begin{array}{c}277->278 \quad 0.67299 \\
{ }^{2} \mathrm{HOMO} \rightarrow{ }^{1} \mathrm{LUMO} \\
(0.67299)\end{array}$ & 3.082 & 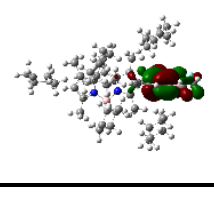 & 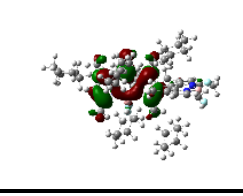 \\
\hline & ${ }^{2} \mathrm{~T}_{1}$ & $\begin{array}{c}277->279 \quad 0.75626 \\
{ }^{2} \mathrm{HOMO} \rightarrow{ }^{2} \mathrm{LUMO} \\
(0.75626)\end{array}$ & 1.042 & & \\
\hline \multirow{3}{*}{200} & $\mathrm{~S}_{1}$ & $\begin{array}{c}276->278 \quad 0.60876 \\
{ }^{1} \mathrm{HOMO} \rightarrow{ }^{1} \mathrm{LUMO} \\
(0.60876)\end{array}$ & 2.726 & & 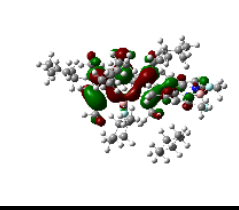 \\
\hline & $\mathrm{CT}$ & $\begin{array}{c}277->278 \quad 0.53816 \\
{ }^{2} \mathrm{HOMO} \rightarrow{ }^{1} \mathrm{LUMO} \\
(0.53816)\end{array}$ & 3.216 & & \\
\hline & ${ }^{2} \mathrm{~T}_{1}$ & $\begin{array}{c}277->279 \quad 0.68878 \\
{ }^{2} \mathrm{HOMO} \rightarrow{ }^{2} \mathrm{LUMO} \\
(0.68878)\end{array}$ & 1.057 & & \\
\hline 300 & $\mathrm{~S}_{1}$ & 277 -> 279 & 2.799 & & \\
\hline
\end{tabular}
B3LYP/cc-pVDZ Level 


$$
\begin{gathered}
{ }^{2} \mathrm{HOMO} \rightarrow{ }^{2} \mathrm{LUMO} \\
(0.60297)
\end{gathered}
$$

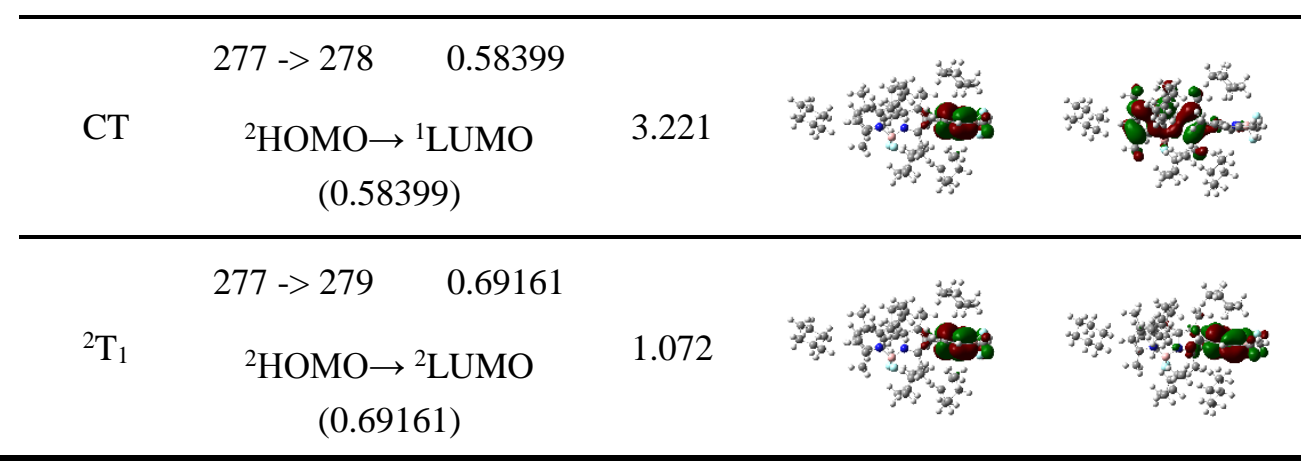

The transition configurations can be expressed as the linear combination of different transitions between the occupied orbitals and unoccupied orbitals in the ground state configuration. In many cases, only one transition configuration takes up a larger proportion. Regarding the identification of the CT state, we can clearly distinguish the transition configuration with the largest proportion in the di-BODIPY system, and the proportion is more than $60 \%$. Thus, we only analyze the transition with the largest proportion of transition configuration in the calculation process. For example:

Excited State 3: $\quad$ Singlet-A $\quad 2.8904 \mathrm{eV} \quad 428.95 \mathrm{~nm} \quad \mathrm{f}=0.0350 \quad\left\langle\mathrm{~S}^{2}>=0.000\right.$

$$
\begin{array}{ll}
306 \rightarrow 308 & 0.19089 \\
307 \rightarrow 308 & 0.67672
\end{array}
$$

This shows that the transition configuration $S_{0} \rightarrow S_{3}$ has a proportion of $91.6 \%$ (i.e. $0.67672^{2} \times 2 \times 100 \%$ ), which can be described as the transition of the doubly occupied orbital 307 to unoccupied orbital 308. Thus, the essence of the $S_{0} \rightarrow S_{3}$ transition can be explained by analyzing this dominant orbital transition configuration. The proportion of other transition configuration $\left(306 \rightarrow 308 \quad 0.19089\right.$, and thus $\left.0.19089^{2} \times 2 \times 100 \%=7.3 \%\right)$ is very small, and it can be ignored.

In order to verify that the identification of the CT state is clear and reliable, we chose some representative configurations and calculate the natural transition orbital distribution and distribution of holes and electrons, as shown in Table S25-S26. The results show that the natural transition orbitals, electron and hole distributions are consistent with molecular orbital transition 
distribution, with obvious CT state characteristics (Table S25 (a) and S26 (a)). We recheck all the transition orbital distributions. Only a few configurations have no obvious CT state characteristics, that is, being the mixed CT and $\mathrm{S}_{1}$ state (Table S25 (b) and S26 (b)). The proportion of such the $\mathrm{CT}$ state in $\mathbf{C H C l}_{3}$ is $6 \%$ and that in c-hex is 5\%. Clearly, the probability is particularly low. Thus, identification of the CT state is unambiguous.

Table S25 (a). The Examples of Electron Transitions of Excited States and Their Corresponding Frontier Molecular Orbitals (HOMO, LUMO) and Natural Transition Orbitals (NBO) and Distribution of Holes and Electrons of di-BODIPY in $\mathrm{CHCl}_{3}$ at the M062X/cc-pVDZ Level.

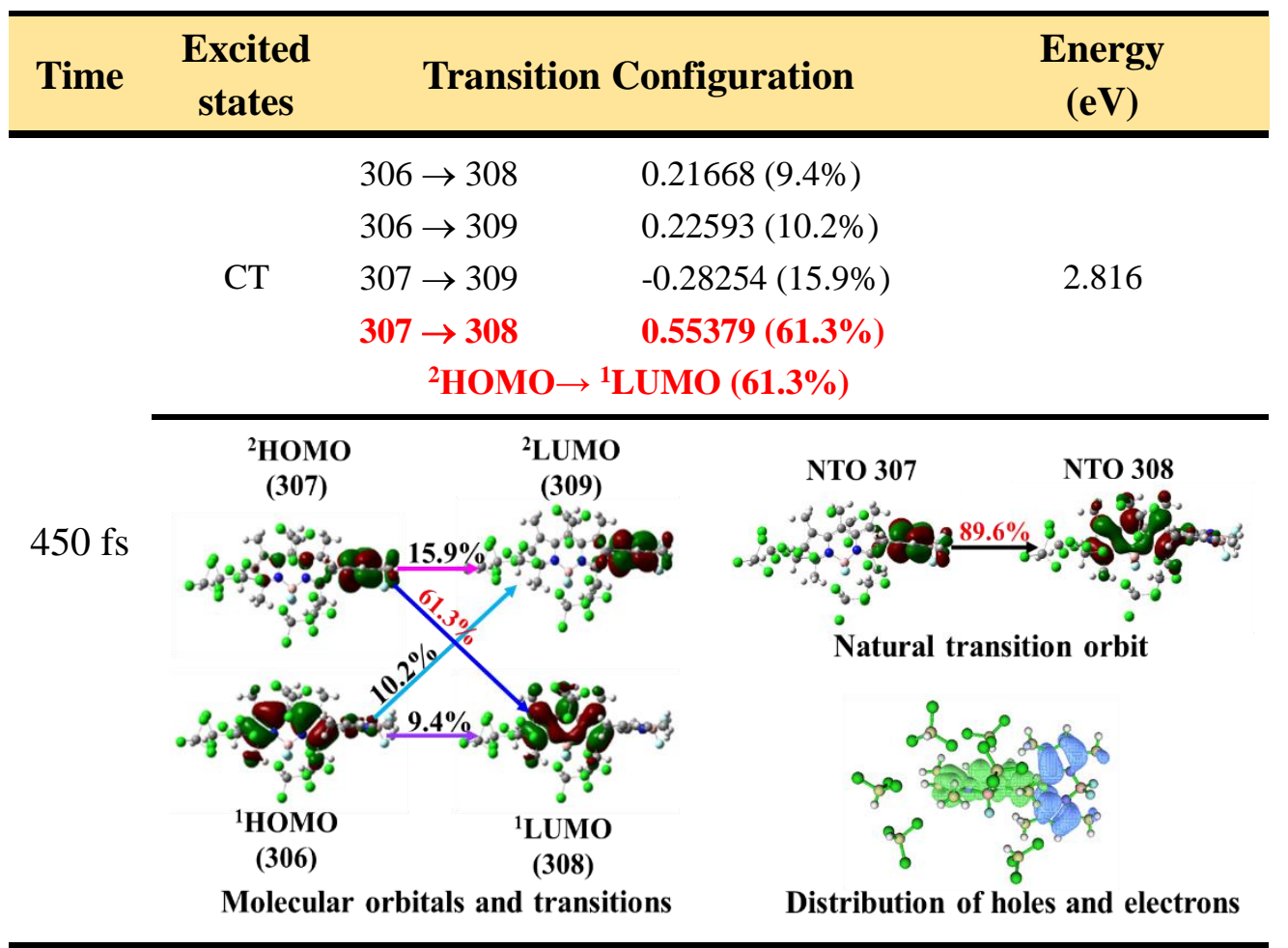




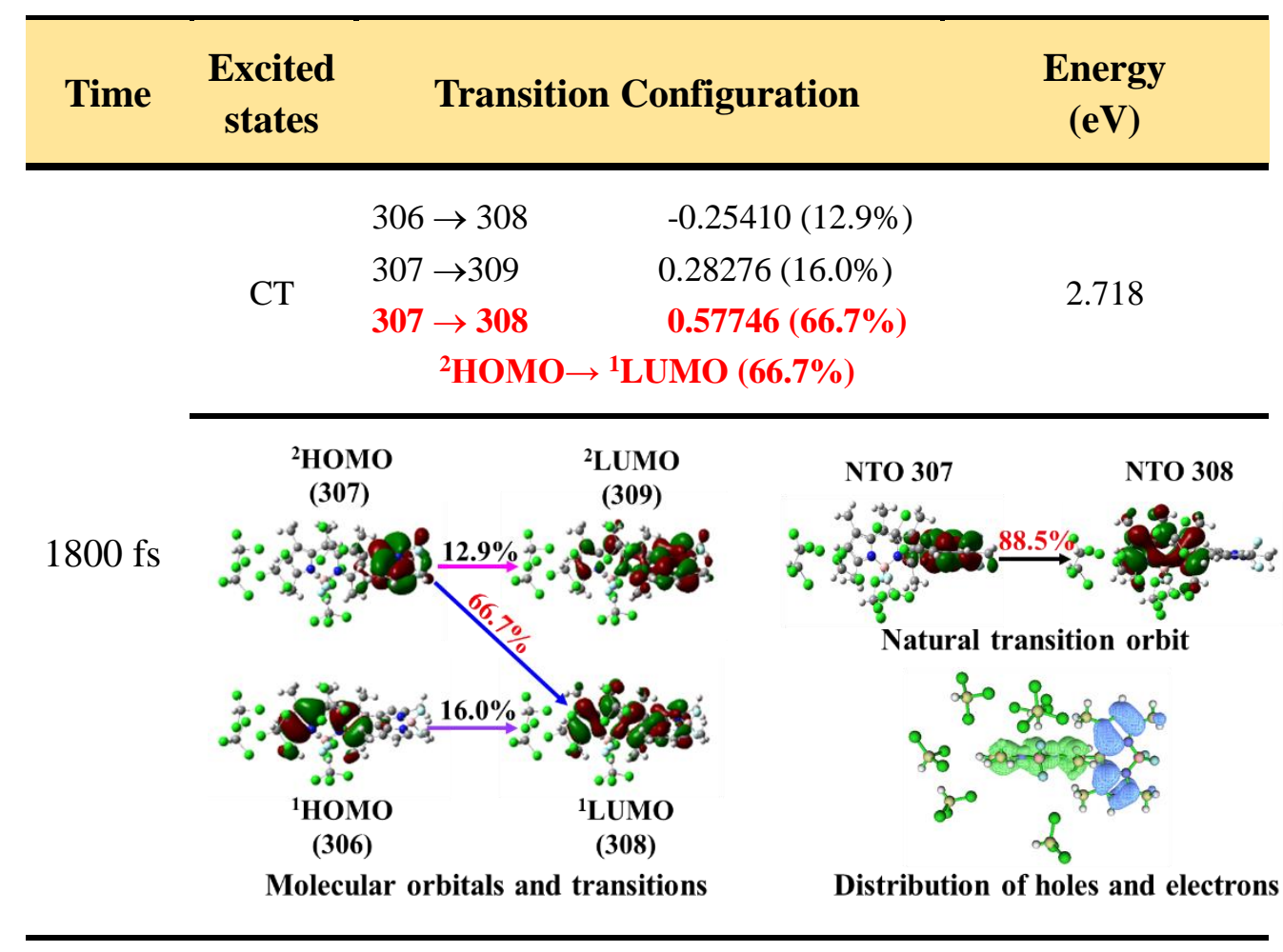

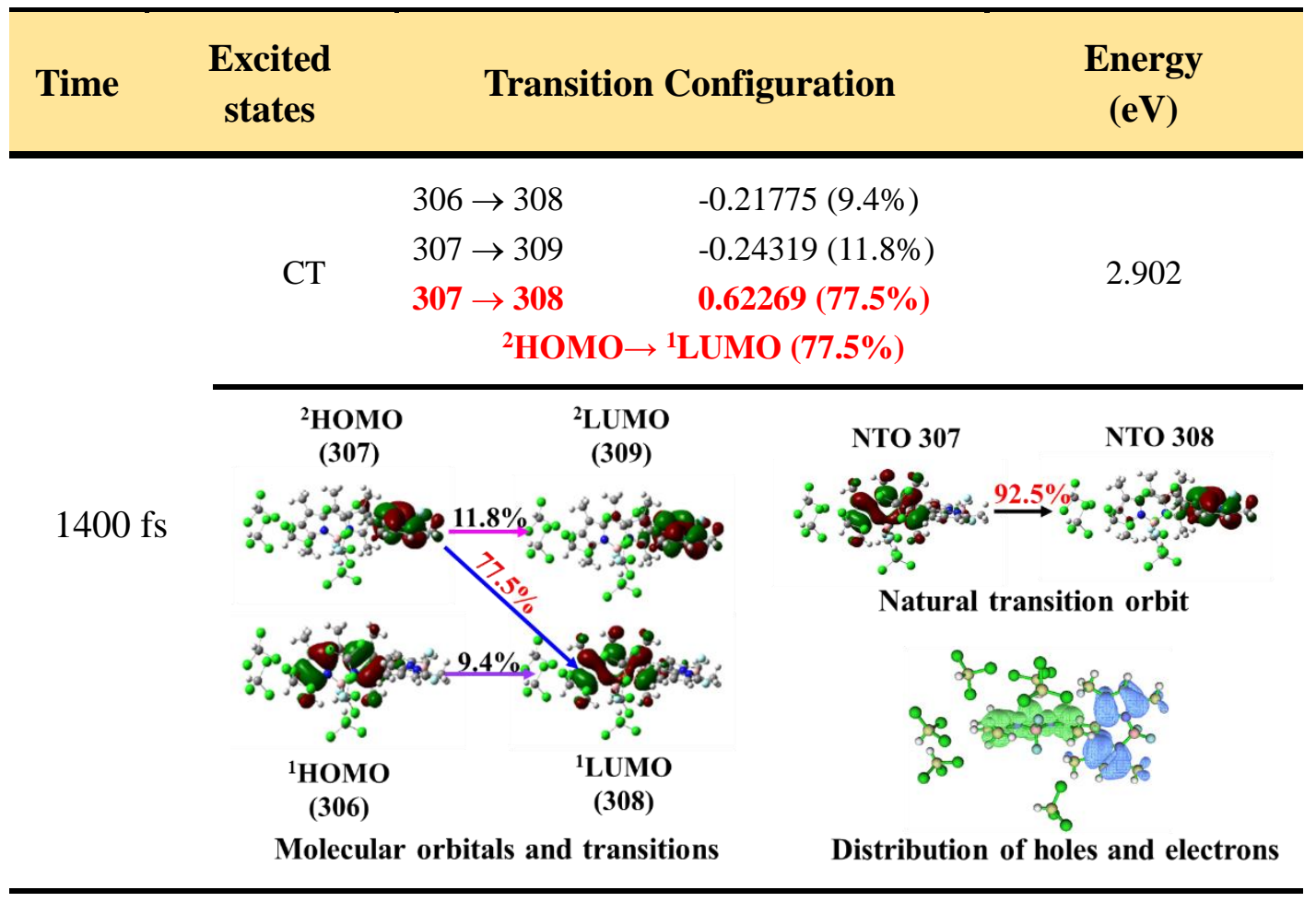




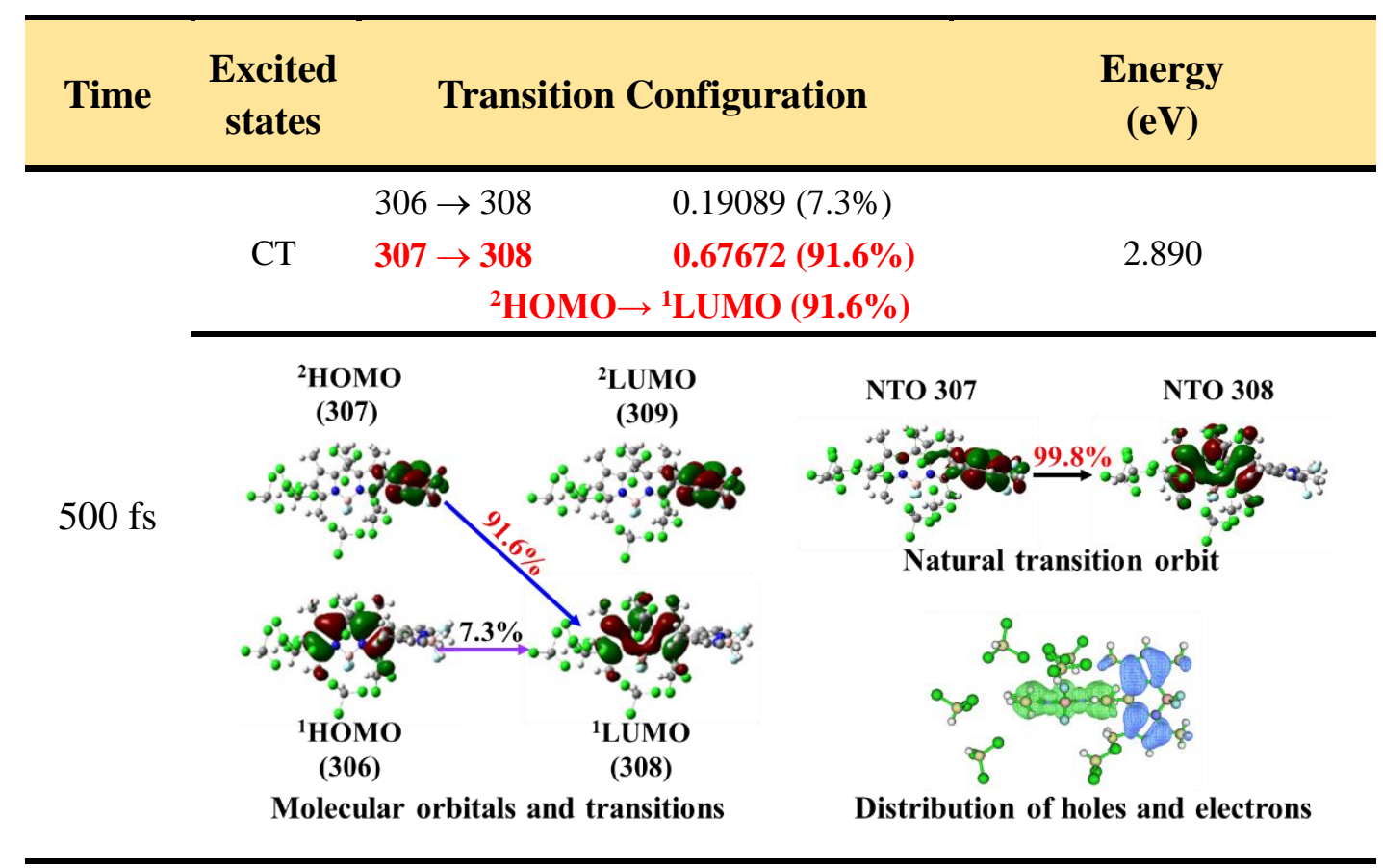

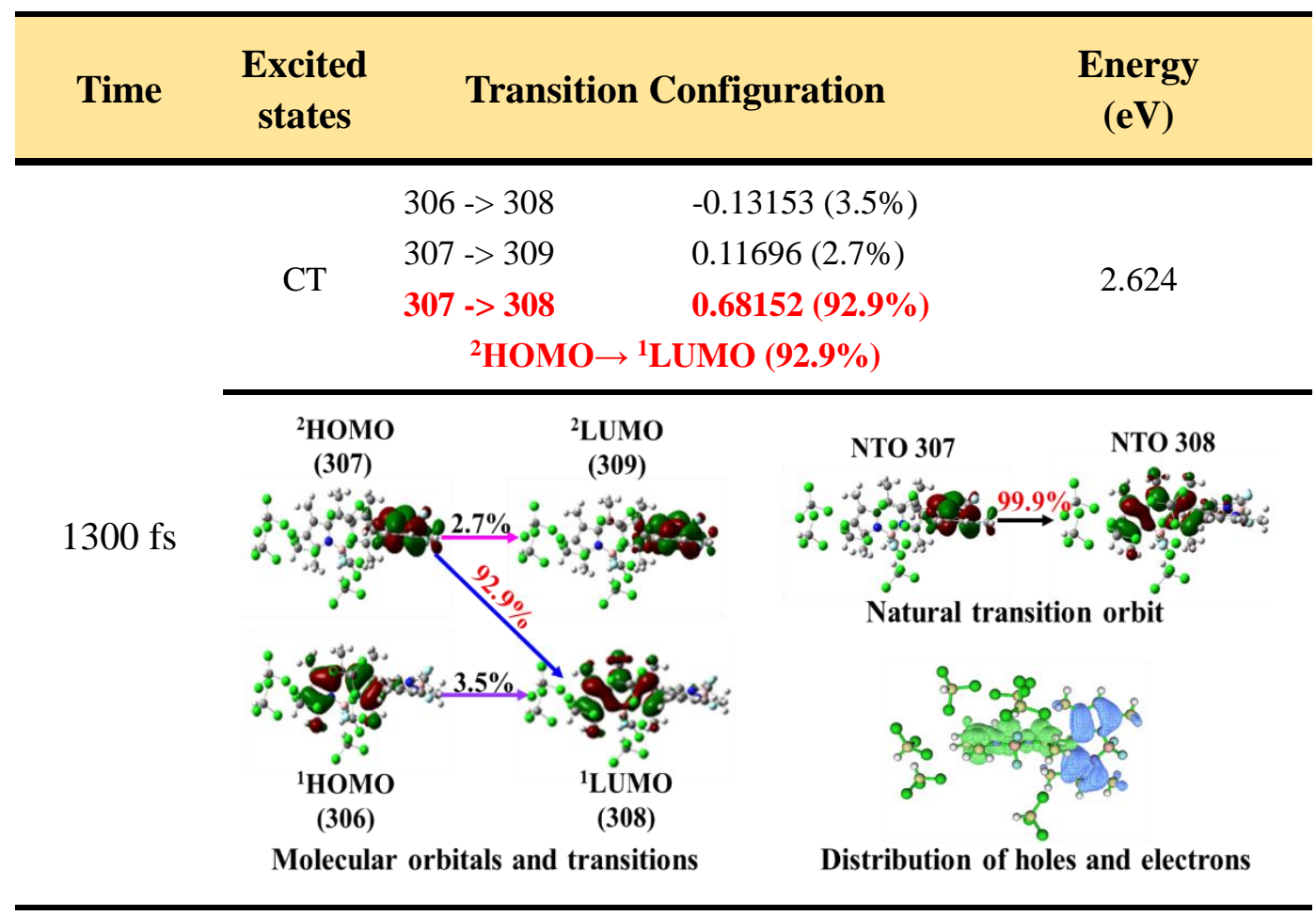


Table S25 (b). The Examples of Electron Transitions of Excited States Which is the S1-Mixed CT State and Their Corresponding Frontier Molecular Orbitals (HOMO, LUMO) and Natural Transition Orbitals (NBO) and Distribution of Holes and Electrons of di-BODIPY in $\mathrm{CHCl}_{3}$ at the M062X/cc-pVDZ Level.

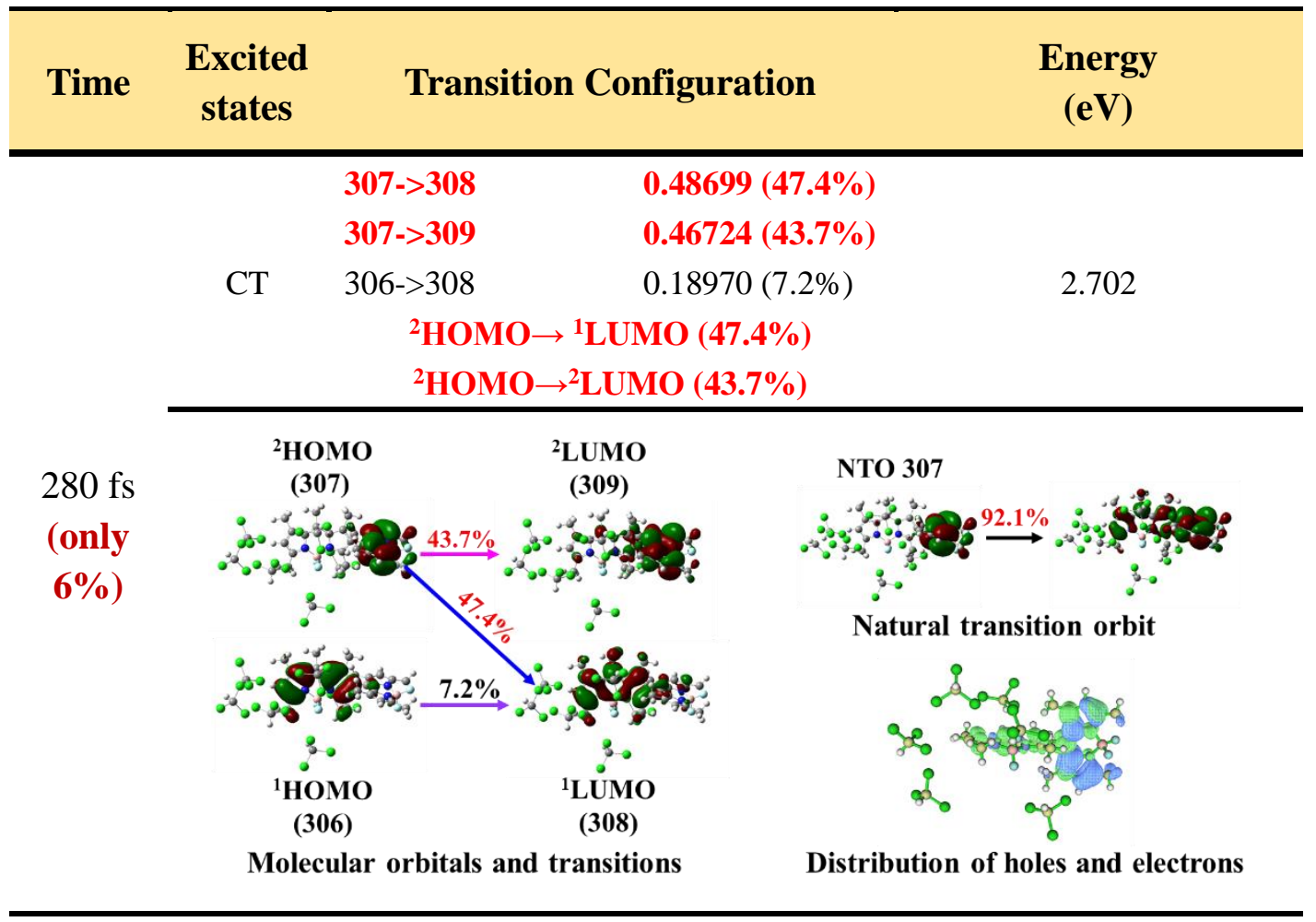


Table S26 (a). The Examples of Electron Transitions of Excited States and Their Corresponding Frontier Molecular Orbitals (HOMO, LUMO) and Natural Transition Orbitals (NBO) and Distribution of Holes and Electrons of di-BODIPY in c-hex at the M062X/cc-pVDZ Level.

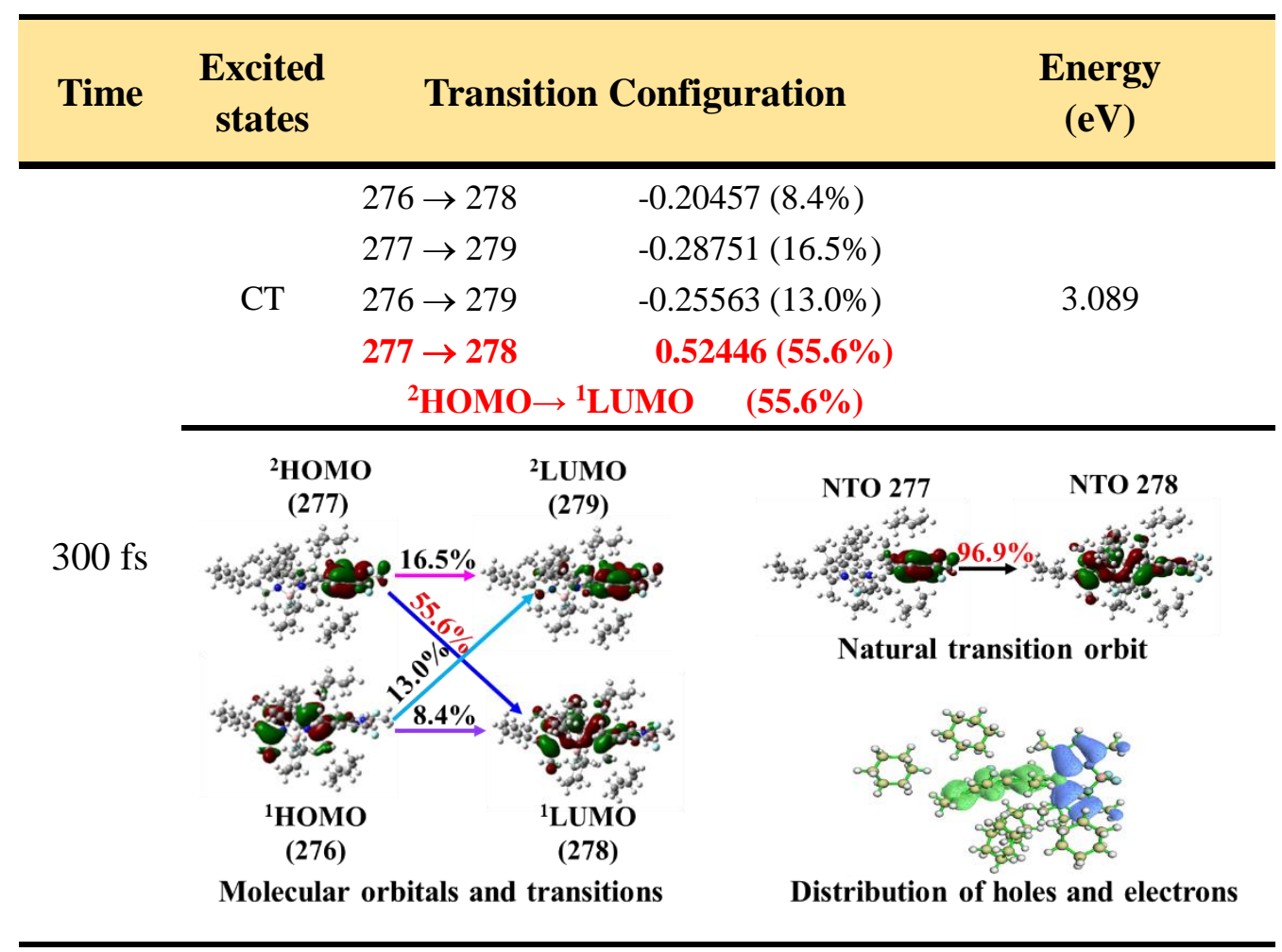

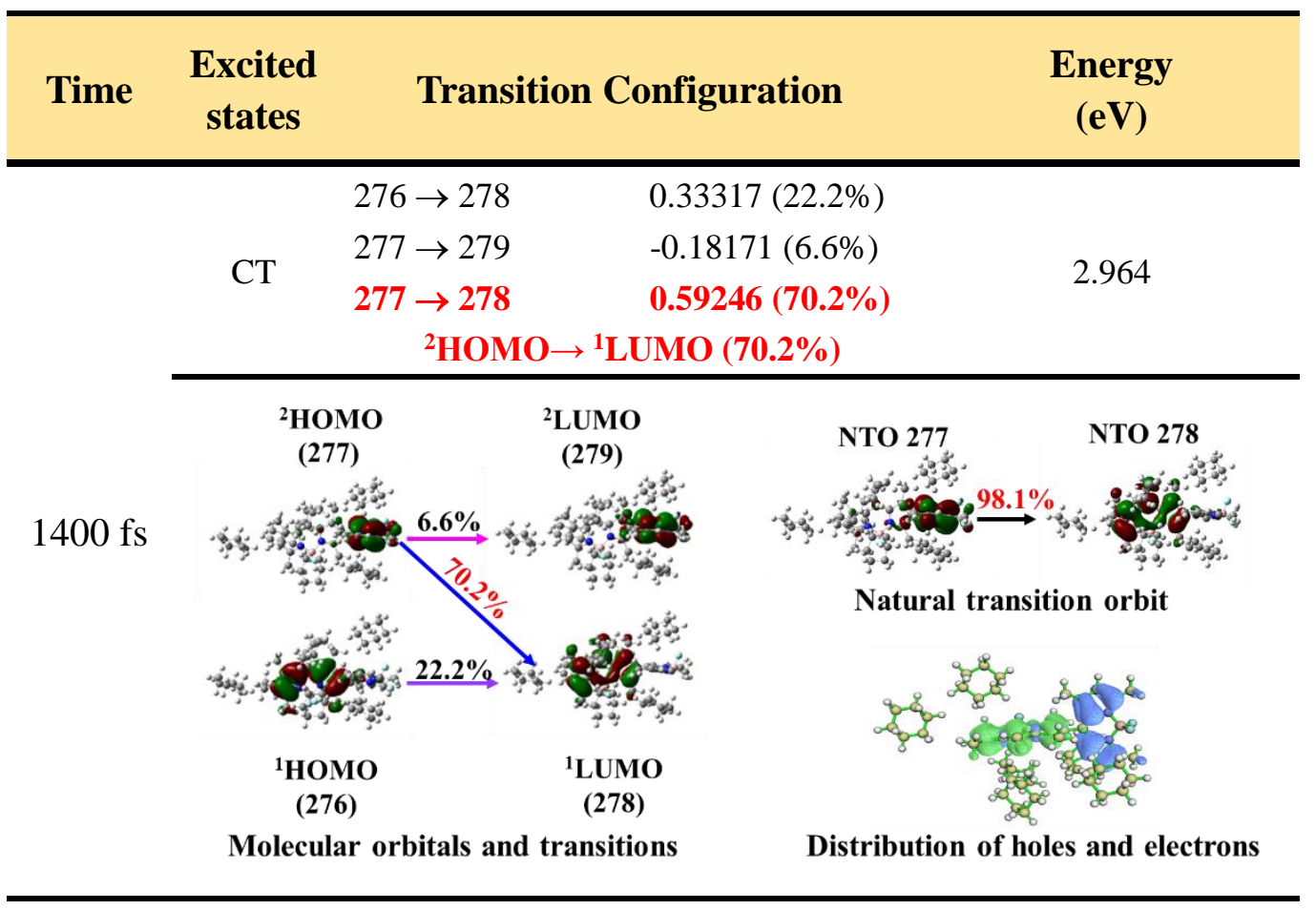




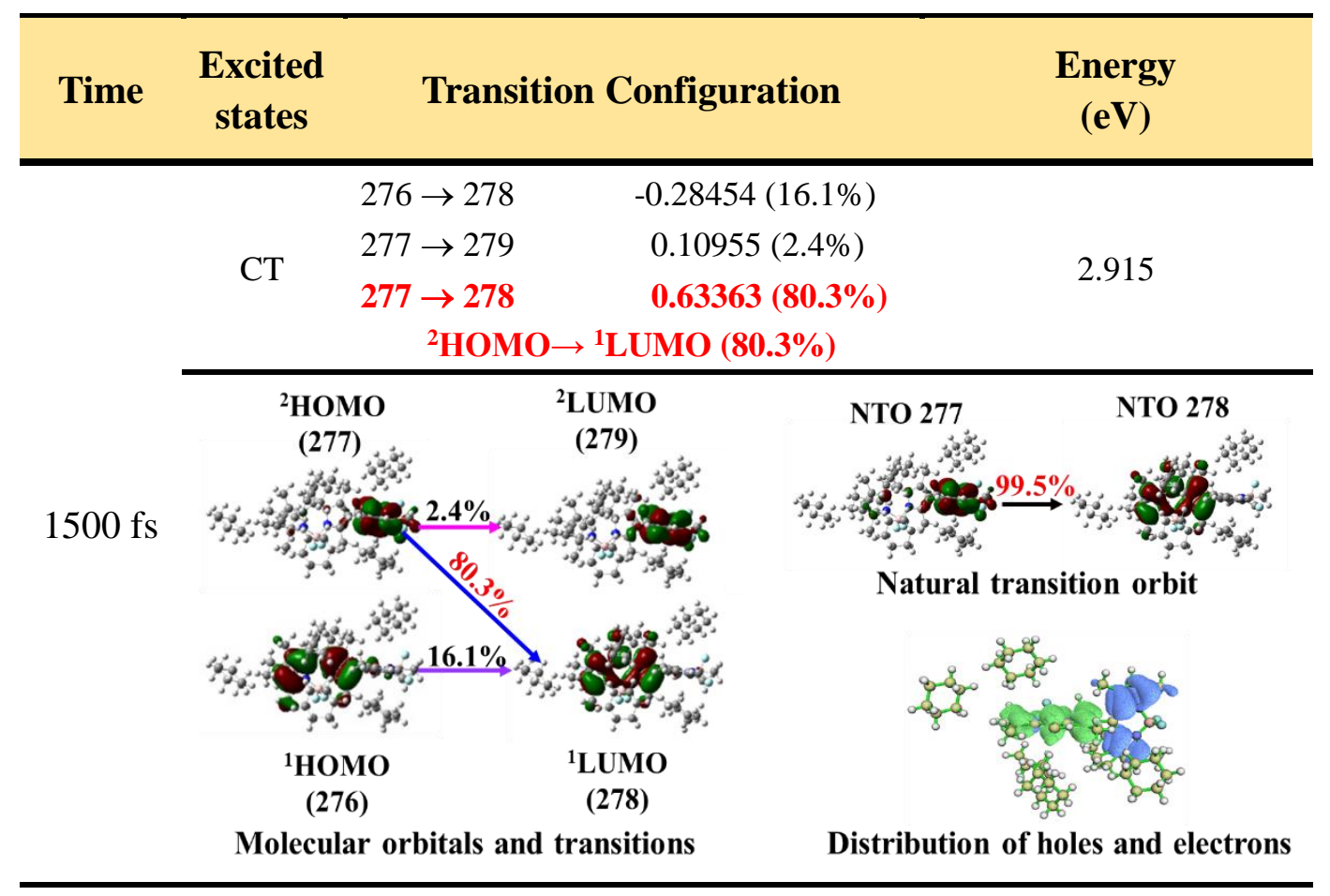

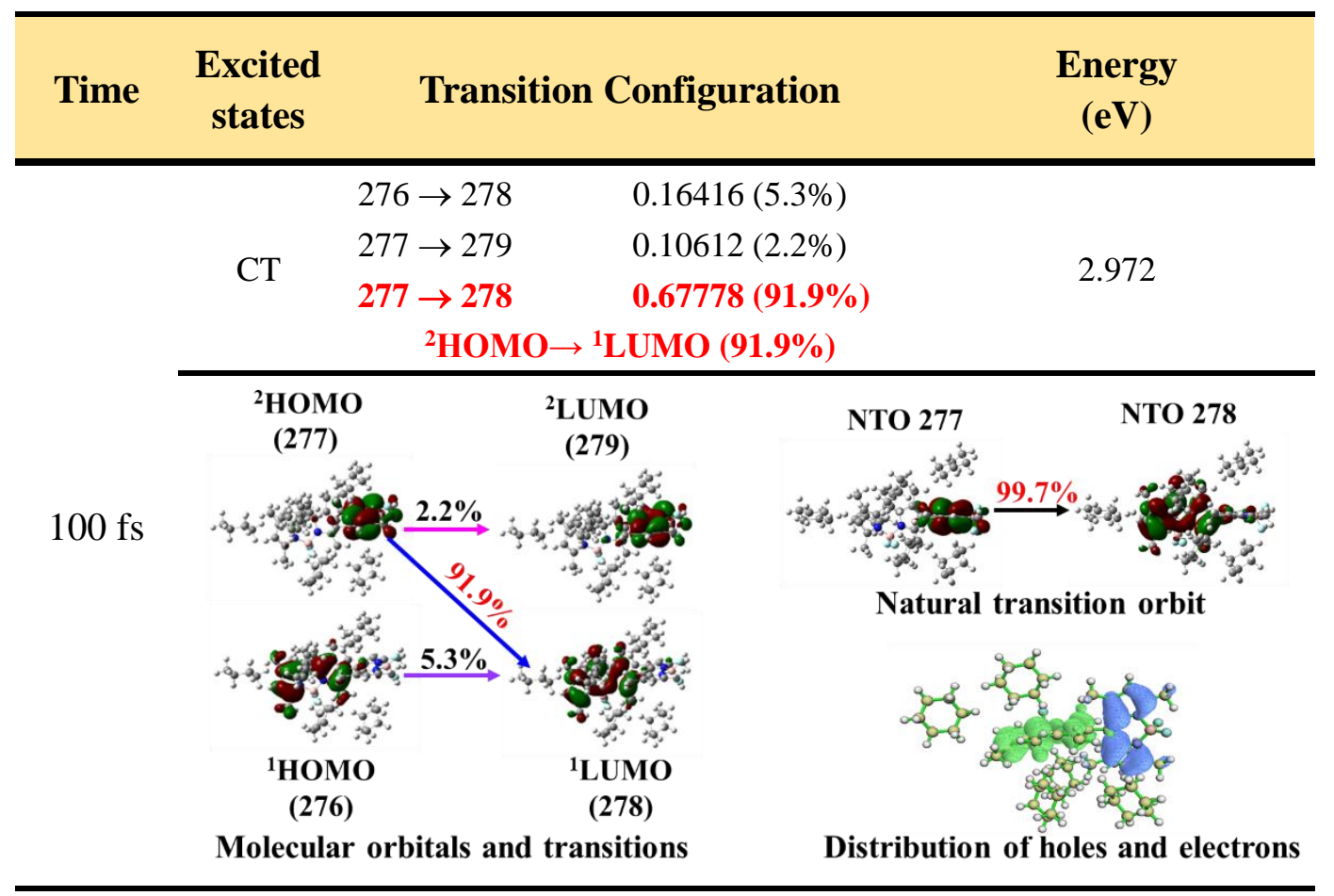


Table S26 (b). The Examples of Electron Transitions of Excited States Which is the $\mathrm{S}_{1}$-Mixed CT state and Their Corresponding Frontier Molecular Orbitals (HOMO, LUMO) and Natural Transition Orbitals (NBO) and Distribution of Holes and Electrons of di-BODIPY and c-hex at the M062X/cc-pVDZ Level.

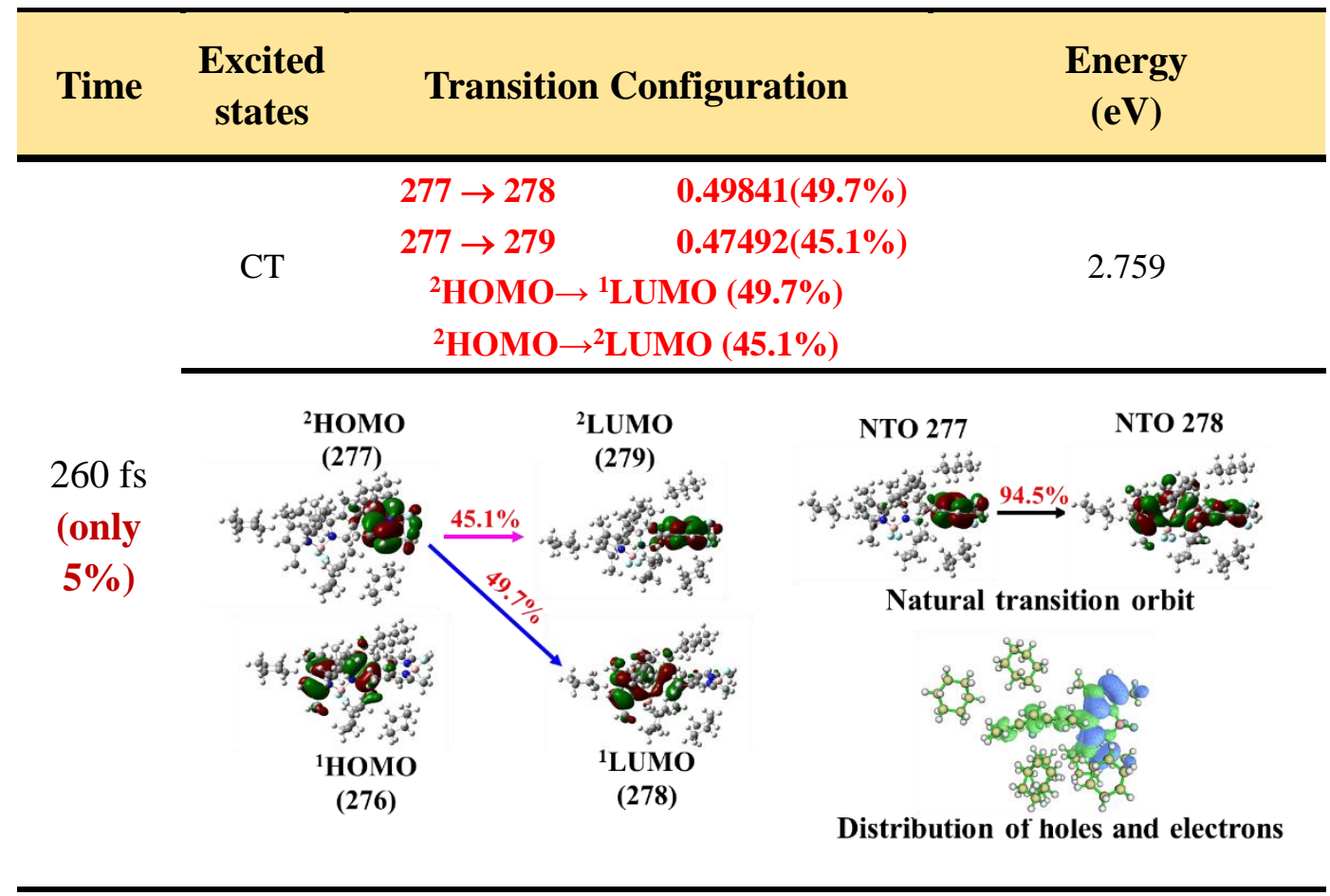

\section{Simple introduction about SF mechanisms and the CT-state role}

As known, the role of CT states is more complicated and the CT states are involved in many processes and mechanisms (e.g. as the virtual states), depending on the systems, as evidenced in literatures. Thus, the study on the CT state role has always been an important topic.

At present, at least two regimes are used to classify the SF mechanisms and for each regime simple limiting cases can be envisaged: (1) coherent versus incoherent process and (2) direct versus indirect process. Their common features are the transfers of two electrons and energy exchange, and their distinctions lie in how to transfer. In the coherent/incoherent regime, if the chromophores are coupled strongly enough and $\mathrm{E}\left(\mathrm{S}_{1}\right)$ approximately equals to $2 \mathrm{E}\left(\mathrm{T}_{1}\right)$ the process could occur coherently on a femtosecond time scale, while if the coupling of the chromophores is weak and/or $\mathrm{E}\left(\mathrm{S}_{1}\right)$ lies too far below $2 \mathrm{E}\left(\mathrm{T}_{1}\right)$, the vibrational relaxation will occur first and the SF will be relatively slow (i.e. the indirect through relaxation with barrier and conical 
intersection). In the direct/indirect regime, the mechanistic distinction is less well defined, depending on the degree of sophistication chosen for the description of the initial and final states. In one imaginable limit, the direct contribution would dominate, and in the other, the mediated contribution would prevail, depending on the stabilization of the possible CT state.

Simply, SF can occur by two general mechanisms: one that directly couples the initial ${ }^{1}\left(\mathrm{~S}_{1} \mathrm{~S}_{0}\right)$ state to a multi-exciton ${ }^{1}\left(\mathrm{~T}_{1} \mathrm{~T}_{1}\right)$ state by a two-electron process and another that indirectly proceeds through a CT state by two consecutive one-electron processes (Smith, M. B. \& Michl, J. Chem. Rev. 2010, 110, 6891; (ref.40) Margulies, E. A. et al Nat Chem 2016, 8, 1120). For the latter, the interactions of the various states involved may make the case more complex, leading the CT state to a real or a virtual CT state in an actual photo-conversion process, depending on the systems.

As for other systems which undergo SF via a real $\boldsymbol{C T}$ mechanism, as concluded in a literature (Teichen, P. E.; Eaves, J. D. JPC B. 2012, 116, 11473) where the authors reported the solventdependent rate expressions for the separate one-electron transfer events, a necessary condition for efficient CT-mediated SF in solvent is that the CT state energies lie in between those of the intramolecular singlet and the multi-exciton triplet-triplet states, i.e., $\mathrm{E}\left(\mathrm{S}_{1} \mathrm{~S}_{0}\right), \mathrm{E}\left(\mathrm{S}_{0} \mathrm{~S}_{1}\right)>\mathrm{E}(\mathrm{CT})>$ $\mathrm{E}\left(\mathrm{T}_{1} \mathrm{~T}_{1}\right)$. This implies that the real $\boldsymbol{C T}$-mediated mechanism should be a representative important type of $\mathbf{S F}$ mechanisms, as revealed in much more references (e.g. ref 25; Johnson, J. C. et al Acc Chem Res 2013, 46, 1290; (ref. 29) Zirzlmeier, J. et al PNAS 2015, 112, 5325; (ref.40) Margulies, E. A. et al. Nat Chem 2016, 8, 1120; (ref.38) Basel, B. S. et al Chem 2018, 4, 1092; (ref.37) Lukman, S. et al Nat Commun, 2016, 7, 13622; Margulies, E. A. et al JASC 2017, 139, 663; Busby, E. et al Nat Mater 2015, 14 , 426; Greyson, E. C. et al JPC B 2010, 114, 14168; Mauck, C. M. ChemPhotoChem 2018, 2, 223; and reference therein and others).

Although we can't point out the real numbers in such a type (real CT-mediated), we could give a simple prediction according to the structural characters of chromophores. Since a SF process needs double electron exchange between two chromophores (intermolecular) or two moieties of a chromophore (intramolecular), the favorable electronic coupling between two parts (e.g. the face-to-face coupling mode or conjugated) encourages the superexchange-like (direct or one-step) mechanisms (involving a concerted two-electron process with a virtual $\boldsymbol{C T}$ state) (e.g. Johnson, J. C. et al Acc Chem Res 2013, 46, 1290; Chen, M. et al JACS 2018, 140, 9184; Burdett, J. J. et al 
JACS 2012, 134, 8597; Miller, C. E. JPC C 2017, 121, 10345; Fuemmeler, E. G. et al ACS Cent. Sci. 2011, 334, 1541; Berkelbach, T. C. et al JCP 2013, 138, 114103). This certainly is a large type of the SF systems, as you pointed out.

But, the weak electronic coupling between two parts (refs.25, 29; Margulies, E. A. et al. Nat Chem 2016, 8, 1120; Basel, B. S. et al Chem 2018, 4, 1092, and those mentioned above, etc) encourages two consecutive one-electron process with a CT intermediate. These real-CT-mediated SF systems, also an important type of the SF systems, involve

a) The unsymmetrical covalent di-BODIPY and its derivatives;

b) Various $\pi$-stacked covalent terrylenediimide dimers;

c) Orthogonal and various other linked oligoacene dimers;

d) Various polymer or oligomer materials of thiophene-1,1-dioxide derivatives;

e) Various 1,3-diphenylisobenzofuran dimers;

f) 3,6-bis(thiophen-2-yl)diketopyrrolopyrrole dimer and other diketopyrrolopyrrole dimer derivatives;

and so on. Of course, different linking or stacking modes of two chromophores could affect the SF mechanisms. In particular, the newly designed systems could be preliminarily examined according to this approach to provide the guide information for further experimental study.

More importantly, as evidenced, the combined effect of solvent and structure dynamics may lead to different SF or photo-physic mechanisms through changing the initial configurations for photoexcitation. That is, some of the dynamic channels are dynamically switched on. Thus, our AIMD sampling configurations could also apply to other types of mechanisms. This expansion needs further investigation.

In short, our present approach (dynamic dual-switched SF channel + AIMD configuration sampling) can reasonably interpret an important type of experimental phenomena about SFs in many systems. In particular, our novelty lies in the dynamic dual-switched channels which are governed by the combined solvent and structural dynamics effect and the characterization method is the AIMD sampling.

That is, our present approach could interpret why a part of systems whose static structure 
don't meet the SF criterion can undergo SF. The reason lies in that there are two barriers $\left(\Delta \mathrm{E}_{\mathrm{SF}}>0, \Delta \mathrm{E}_{\mathrm{CT}-\mathrm{S} 1}>0\right)$ to inhibit the $\mathrm{SF}$ in the static structure and gas phase. Our approach proves there are sizable probabilities to remove the two barriers through AIMD samplings, establishing the CT state dynamics or dual-switcher $\left(\Delta \mathrm{E}_{\mathrm{SF}}, \Delta \mathrm{E}_{\mathrm{CT}-\mathrm{S} 1}\right)$ dynamics and their association with the SF phenomena.

In fact, the virtual CT state for SF is not only that the energy of CT state is higher than that of $S_{1}$ state, but more importantly, there must be a coupling between the states (such as CT state and $\mathrm{S}_{1}$ state or between CT state and ${ }^{1} \mathrm{TT}$ state), which can be revealed by the electron transitions of the excited states and their corresponding frontier molecular orbital distributions.

By considering these detailed comments, to clarify the mediating role of the virtual CT states in assisting SF, we carefully examine the virtual CT states including their energetics and transition frontier molecular orbitals and natural orbitals of the sampling configurations with $\Delta \mathrm{E}_{\mathrm{CT}-\mathrm{S} 1}>0$ in our simulations. Interestingly, the virtual CT states exhibit different mixings by the $S_{1}$ state in a certain energy population of the CT states, forming the $S_{1}$-mixed CT states with the corresponding transition energies and orbital distributions over two monomers, and thus promote or realize the SF. The orbital or state mixing degree depends on the chromophore structure dynamics and solvent properties which jointly govern the proximity of the orbital energies of two moieties. In general, when the CT state is lower than the $\mathrm{S}_{1}$ state, the CT state acts as a real CT state to realize SF, as mentioned in the text. When the CT state is slightly higher than the $S_{1}$ state in some sampling configurations, the CT state actually is a mixed CT state with the $S_{1}$ state, featuring the transition orbitals distributing over the two monomers of diBODIPY in solvents or transition being a mixed transition of $\mathrm{S}_{1}$ and CT. Although $\Delta \mathrm{E}_{\mathrm{CT}-\mathrm{S} 1}>0$ in such configurations, the SF could occur through the $\mathrm{S}_{1} / \mathrm{CT}$-mixing, which can interpret the possible SF phenomena for the virtual CT mediated SF case. However, not all CT states can assist to realize the SF. Examinations indicate that when the CT state is energetically quite higher than the $S_{1}$ state, they don't mix with each other. In this case, the CT state actually is a barrier state which inhibits the SF. Thus, we can know how high the virtual CT state in energy $\left(\Delta \mathrm{E}_{\mathrm{CT}-\mathrm{S} 1}=\delta>0\right)$ does not mediate the SF through examining if the CT is mixed by the $\mathrm{S}_{1}$ state. If the CT is $\mathrm{S}_{1}$-mixed, the virtual CT state could mediate the SF, whereas if the CT is not mixed 
by $\mathrm{S}_{1}$, the CT state is a barrier state, inhibiting the SF. Certainly, further examining the transition energy of the mixed CT state, we can calculate $\Delta \mathrm{E}_{\mathrm{CT}-\mathrm{S} 1}=\delta(\delta>0)$.

In detail, for the di-BODIPY system, no matter in $\mathrm{CHCl}_{3}$ or in c-hex solvent, the frontier molecular orbitals (HOMO, LUMO) for transition show that the $\mathrm{S}_{1}$ and CT state almost all are pure states for 200 sampling configurations with the probabilities of $94 \%$ in $\mathrm{CHCl}_{3}$ and $95 \%$ in c-hex, respectively. Only a few configurations have the $S_{1}$-mixed virtual CT state with the probabilities of $6 \%$ in $\mathrm{CHCl}_{3}$ and $5 \%$ in c-hex. Thus, for the di-BODIPY system, the possibilities of real CT state (i.e. the pure CT state, $\mathrm{E}\left(\mathrm{S}_{1}\right)>\mathrm{E}(\mathrm{CT})$ ) are very high in both solvents (94\% in $\mathrm{CHCl}_{3}$ and $95 \%$ in c-hex), while the possibilities of the $\mathrm{S}_{1}$-mixed virtual CT state (i.e. $\left.\mathrm{E}\left(\mathrm{S}_{1}\right) \leq \mathrm{E}(\mathrm{CT})\right)$ are only $6 \%$ in $\mathrm{CHCl}_{3}$ and $5 \%$ in c-hex, respectively. Furthermore, we calculate $\Delta \mathrm{E}_{\mathrm{CT} \text {-S1 }}$ of all the configurations with the $\mathrm{S}_{1}$-mixed CT state, as shown in Figure $\mathrm{S} 19$ (a). The upper limits of $\Delta \mathrm{E}_{\mathrm{CT}-\mathrm{S} 1}$ are $0.096 \mathrm{eV}$ in $\mathrm{CHCl}_{3}$ and $0.127 \mathrm{eV}$ in c-hex for the sampling configurations with the $\mathrm{S}_{1}$-mixed virtual CT state, respectively. Thus, we could define $\Delta \mathrm{E}_{\mathrm{CT} \text {-S1 }}$ $<\delta=0.1 \mathrm{eV}$ in $\mathrm{CHCl}_{3}$ and $\Delta \mathrm{E}_{\mathrm{CT}-\mathrm{S} 1}<\delta=0.13 \mathrm{eV}$ in c-hex as the criteria for the virtual CT-mediated SF. Undoubtedly, the upper limit values are different, strongly depending on the chromophore structures, properties and dynamics and solvent properties.

Combining the thermodynamic criterion $\left(\Delta \mathrm{E}_{\mathrm{SF}} \leq 0\right)$, as shown in Figure $\mathrm{S} 19$ (b), the probability of $\Delta \mathrm{E}_{\mathrm{SF}} \leq 0$ and $\Delta \mathrm{E}_{\mathrm{CT}-\mathrm{S} 1} \in(0,0.1 \mathrm{eV}]$ is only $5 \%$ in $\mathrm{CHCl}_{3}$ that can undergo $\mathrm{SF}$ via a virtual-CT assisted pathway in all 200 sampling configurations, while that of $\Delta \mathrm{E}_{\mathrm{SF}} \leq 0$ and $\Delta \mathrm{E}_{\mathrm{CT}}$ $\mathrm{S} 1<0.13 \mathrm{eV}$ is only $2.5 \%$ in c-hex for the virtual CT-mediated SF (Figure S19 (c)). This analysis indicates that the possibility of SF in c-hex is very low even if a virtual CT assisted pathway is considered. This is because the combined structure dynamics and solvent effect can't lower the CT state in c-hex to be an effective degree for realizing the mixing with $S_{1}$ state. Thus, the extended analysis by considering possible virtual CT-mediated pathway still interprets well the experimental observation that di-BODIPY in c-hex doesn't undergo SF.

In short, the above analysis indicates that the di-BODIPY in both solvents undergoes the real CT mediated SF, and the virtual CT mediated SF channel hardly plays any role. 

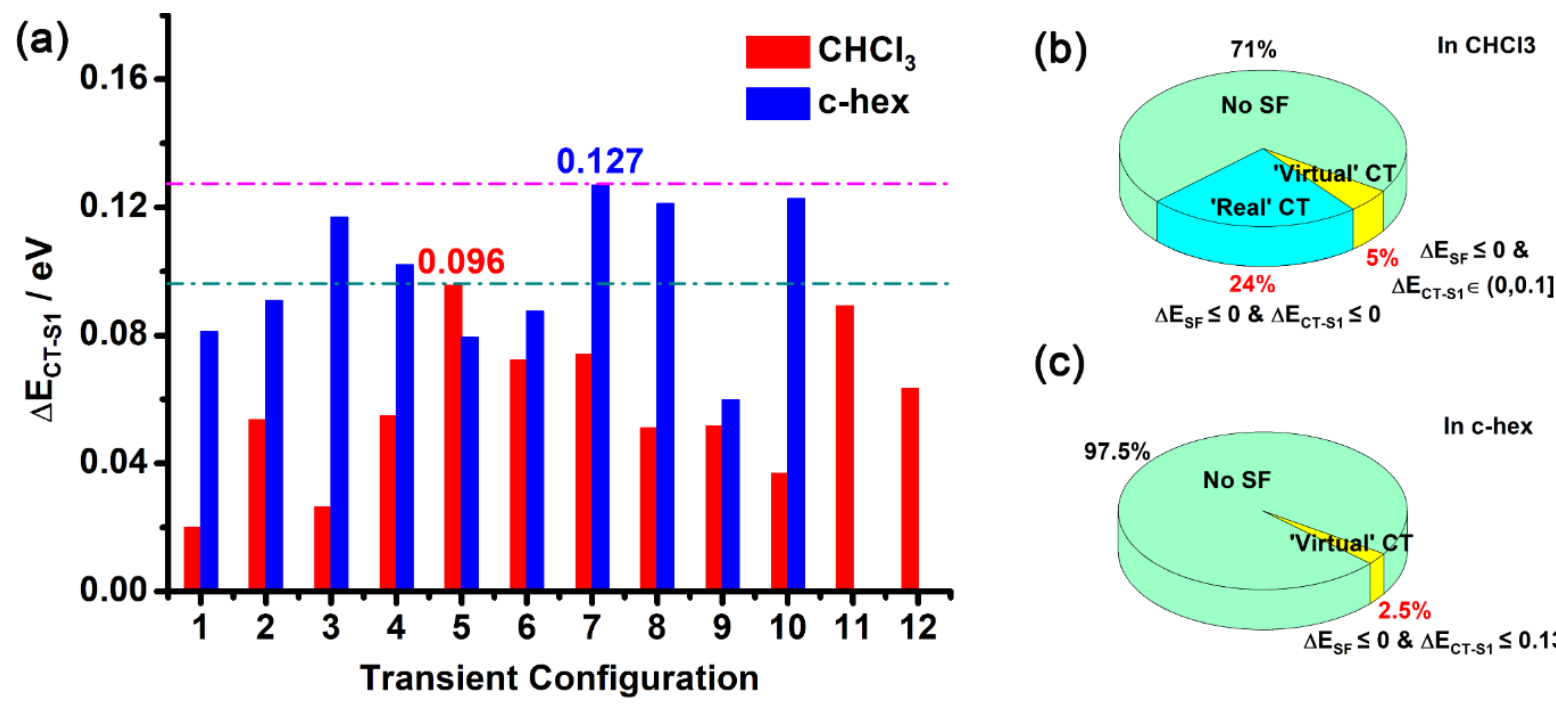

(c)

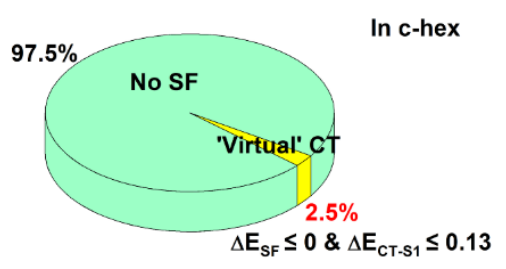

Figure S19. (a) $\Delta \mathrm{E}_{\mathrm{CT}-\mathrm{S} 1}$ of the configurations which $\mathrm{CT}$ and $\mathrm{S}_{1}$ state are mixed. (b) The probability statistics according to the $\Delta \mathrm{E}_{\mathrm{SF}}$ and $\Delta \mathrm{E}_{\mathrm{CT}-\mathrm{S} 1}$ criteria for 200 transient geometries in $\mathrm{CHCl}_{3}$ and c-hex.

\section{Further Verification of the Virtual CT-Mediated SF in Other Systems Using Our Approach:}
1) DP-Mes in toluene (from Ref.37);
2) DT-Mes in ethanol and toluene (from Ref.36)

Static Structures in Solvents: To further clarify the mediating role of the virtual CT state, using our approach, we also examine other systems which were reported in (Refs.36,37). The calculated excited states and their corresponding frontier molecular orbital distributions of DPMes (Ref. 37) and DT-Mes (Ref. 36) with the virtual CT States are shown in Table S27-S29. Examination of the transition orbital distributions reveals that for the two systems in different solvents, their CT states are not the pure CT state, instead the strongly mixed states of CT state with $\mathrm{S}_{1}$ state. Thus, they could undergo SF via a virtual CT-mediated mechanism. It should be noted that for different systems in different solvents, $\Delta \mathrm{E}_{\mathrm{CT}-\mathrm{S} 1}$ (here $\mathrm{CT}$ actually is the $\mathrm{S}_{1}$-mixed CT state) are different: $\Delta \mathrm{E}_{\mathrm{CT}-\mathrm{S} 1}=\delta=0.114 \mathrm{eV}$ for DP-Mes in toluene

$$
\begin{aligned}
& \Delta \mathrm{E}_{\mathrm{CT}-\mathrm{S} 1}=\delta=0.032 \mathrm{eV} \text { for DT-Mes in ethanol } \\
& \Delta \mathrm{E}_{\mathrm{CT}-\mathrm{S} 1}=\delta=0.071 \mathrm{eV} \text { for DT-Mes in toluene }
\end{aligned}
$$

Clearly, it is impossible to estimate an united criterion $\Delta \mathrm{E}_{\mathrm{CT}-\mathrm{S} 1} \leq \delta$ to judge if SF can occur through the real CT-mediated or virtual CT-mediated channel for lots of systems because the threshold ( $\delta$ ) strongly depends on the structures (including the dynamic structures) and properties of 
chromophore and solvent properties. That is, that if the CT state mediates SF depends on if there is a real CT state or a virtual CT state which couples with the $\mathrm{S}_{1}$ state. But, it is easy to determine the $\delta$ value for a system (including chromophore and solvent) through checking the transition orbital distributions and corresponding transition energies (Table S27-S29).

The Modified Criteria: To make our model more general, we thus extend the proposed dualswitched dynamic SF channel by considering the contribution of the mixed CT states. The general rule (for Switch 2) is outlined as:

1) $\Delta \mathrm{E}_{\mathrm{SF}} \leq 0$ and $\Delta \mathrm{E}_{\mathrm{CT}-\mathrm{S} 1} \leq 0$, SF via a real $\mathrm{CT}$ mediated channel;

2) $\Delta \mathrm{E}_{\mathrm{SF}} \leq 0$ and $\Delta \mathrm{E}_{\mathrm{CT}-\mathrm{S} 1}>0$ with a $\mathrm{S}_{1}$-mixed $\mathrm{CT}$ state, $\mathrm{SF}$ via a virtual $\mathrm{CT}$ mediated channel;

3) $\Delta \mathrm{E}_{\mathrm{SF}} \leq 0$ and $\Delta \mathrm{E}_{\mathrm{CT}-\mathrm{S} 1}>0$ without a $\mathrm{S}_{1}$-mixed $\mathrm{CT}$ state, no $\mathrm{SF}$.

AIMD Simulation Verification: To further verify the extended criteria, we also perform AIMD simulation on DT-Mes in ethanol (the SF system in Ref. 36) using the same method because two types of the CT states (real versus virtual) are observed in this DT-Mes system in the structure dynamics. Examination indicates that for this system there are the real CT states in some sampling configurations, there are the mixed virtual CT states featuring delocalized transition orbital distribution in some other sampling configurations, and there are the pure virtual CT states in the remaining sampling configurations, as shown in Table S30-S32. For example, (1) two representative configurations, their $\Delta \mathrm{E}_{\mathrm{SF}} \leq 0$ and $\Delta \mathrm{E}_{\mathrm{CT}-\mathrm{S} 1} \leq 0$, and thus DT-Mes in these two configurations in ethanol can undergo SF via a real CT mediated channel (Table S30);

(2) the other two representative configurations, their $\Delta \mathrm{E}_{\mathrm{SF}} \leq 0$ and $\Delta \mathrm{E}_{\mathrm{CT}-\mathrm{S} 1}>0\left(\Delta \mathrm{E}_{\mathrm{CT}-\mathrm{S} 1}=0.075\right.$ $\mathrm{eV}$ and $0.109 \mathrm{eV}$, respectively) and both configurations have the $\mathrm{S}_{1}$-mixed CT states, and thus DT-Mes in these two configurations in ethanol can undergo SF via a virtual CT mediated channel (Table S31);

(3) the third case (two representative configurations), their $\Delta \mathrm{E}_{\mathrm{SF}} \leq 0$ and $\Delta \mathrm{E}_{\mathrm{CT}-\mathrm{S} 1}>0$ but without any mixed CT states, and thus DT-Mes in ethanol doesn't undergo SF at these configurations (Table S32).

Clearly, these representative results indicate that our approach combining the dual-switched dynamic channel and AIMD samplings and singlet pre-fission (pre-SF) energetics analysis can 
reasonably predict the SF possibility of a system in media and its SF channel (the real CT mediated versus the virtual $\mathrm{CT}$ mediated). The threshold $\delta\left(\Delta \mathrm{E}_{\mathrm{CT}-\mathrm{S} 1} \leq \delta\right)$ strongly depends on the system (including the structure and dynamics, medium) and can be easily determined by checking the transition orbital distributions and corresponding transition energies.

Certainly, extension of the present approach including dynamics samplings and calculations of transition orbital distributions and energetics to other mechanisms needs further investigations and relevant work is underway.

Table S27. Electron Transitions of Excited States and Their Corresponding Frontier Molecular Orbitals for DP-Mes in Toluene with Virtual CT State $\left(\Delta \mathrm{E}_{\mathrm{SF}}=-0.227 \mathrm{eV}, \Delta \mathrm{E}_{\mathrm{CT}-\mathrm{S} 1}=0.114 \mathrm{eV}\right)$

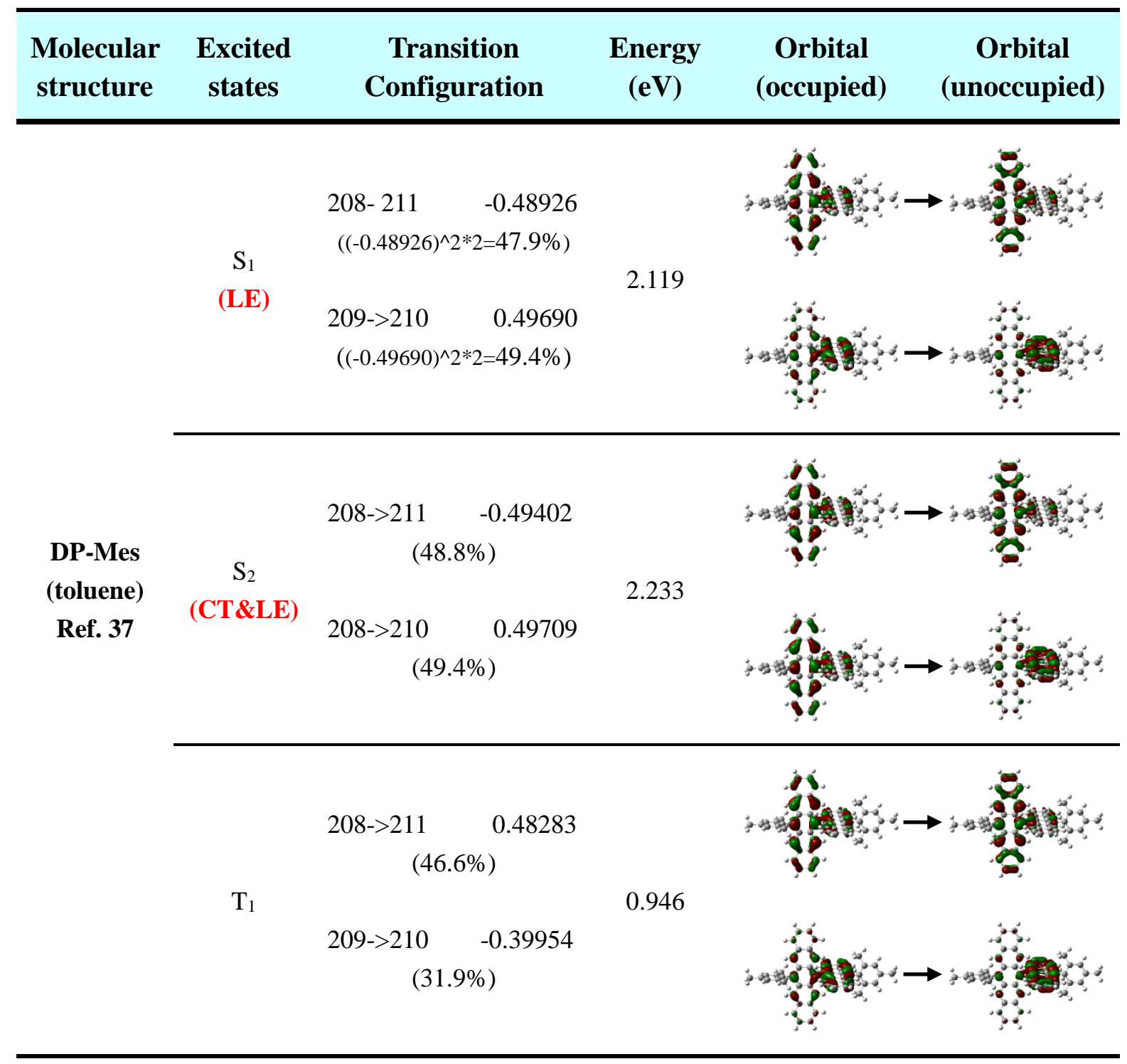


Table S28. Electron Transitions of Excited States and Their Corresponding Frontier Molecular Orbitals for DT-Mes in Ethanol with Virtual CT State $\left(\Delta \mathrm{E}_{\mathrm{SF}}=-0.04 \mathrm{eV}, \Delta \mathrm{E}_{\mathrm{CT}-\mathrm{S} 1}=0.032 \mathrm{eV}\right)$

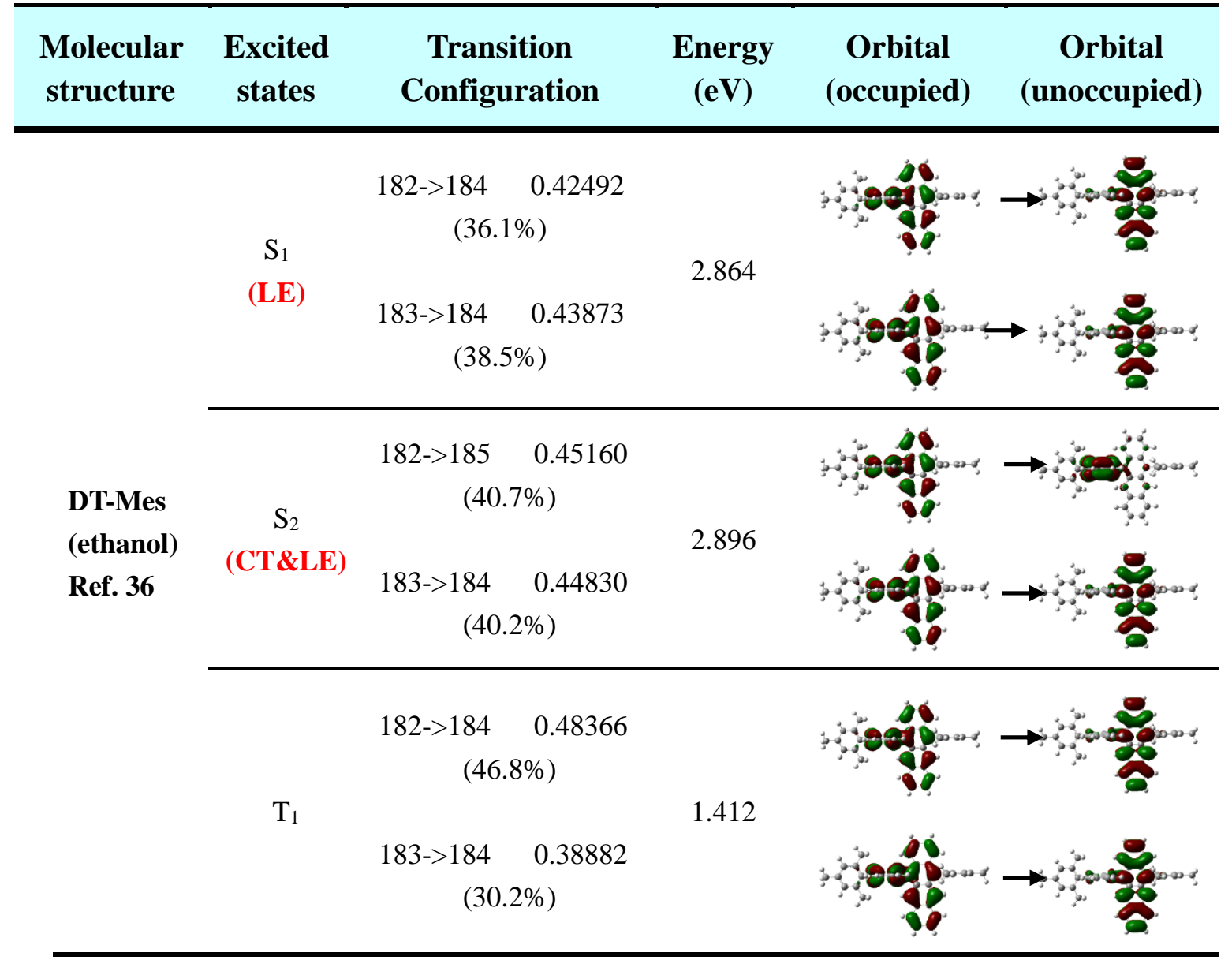


Table S29. Electron Transitions of Excited States and Their Corresponding Frontier Molecular Orbitals for DT-Mes in Toluene with Virtual CT State $\left(\Delta \mathrm{E}_{\mathrm{SF}}=-0.058 \mathrm{eV}, \Delta \mathrm{E}_{\mathrm{CT}-\mathrm{S} 1}=0.071 \mathrm{eV}\right)$

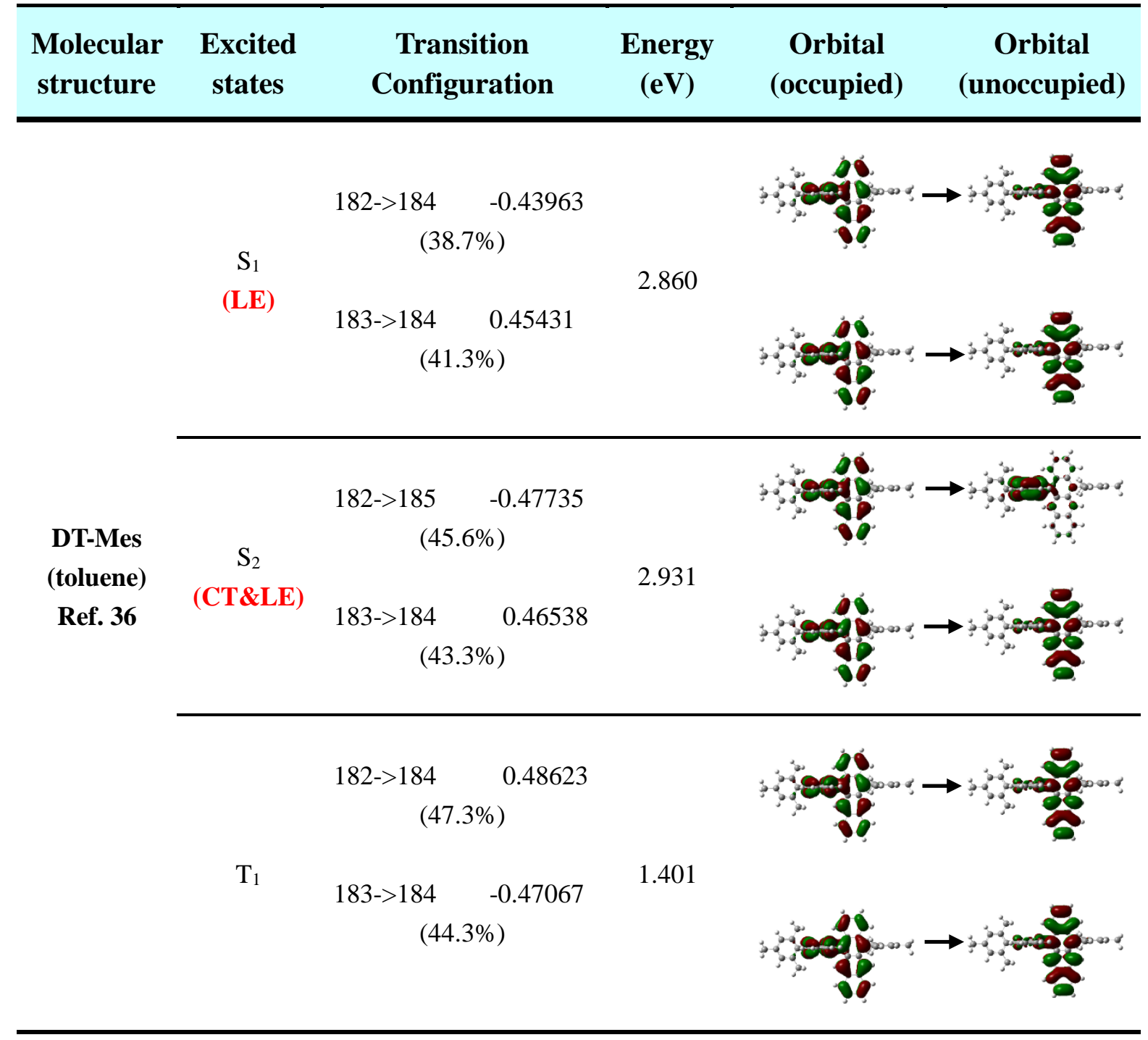


Table S30. The Examples of Electron Transitions of Excited States and Their Corresponding Frontier Molecular Orbitals for DT-Mes in Ethanol with Real CT state $\left(\Delta \mathrm{E}_{\mathrm{SF}}<0, \Delta \mathrm{E}_{\mathrm{CT}-\mathrm{S} 1}<0\right)$

\begin{tabular}{|c|c|c|c|c|c|c|}
\hline $\begin{array}{c}\text { Time/ } \\
\text { fs }\end{array}$ & $\begin{array}{c}\text { Excited } \\
\text { states }\end{array}$ & \multicolumn{2}{|c|}{$\begin{array}{c}\text { Transition } \\
\text { Configuration }\end{array}$} & $\begin{array}{c}\text { Energy } \\
(\mathrm{eV})\end{array}$ & $\begin{array}{c}\text { Orbital } \\
\text { (occupied) }\end{array}$ & $\begin{array}{c}\text { Orbital } \\
\text { (unoccupied) }\end{array}$ \\
\hline \multirow{5}{*}{300} & CT & $\begin{array}{l}260->262 \\
260->263 \\
261->262\end{array}$ & $\begin{array}{l}0.21331 \\
0.12528 \\
0.65965\end{array}$ & 2.630 & 3 & sats \\
\hline & & \multicolumn{2}{|c|}{$(87.0 \%)$} & & & \\
\hline & $\begin{array}{c}\mathrm{S}_{1} \\
(\mathrm{LE})\end{array}$ & $\begin{array}{l}260->263 \\
261->262 \\
261->263 \\
260->262\end{array}$ & $\begin{array}{r}0.20418 \\
-0.23988 \\
-0.10116 \\
0.61352\end{array}$ & 2.889 & & $\because$ \\
\hline & & \multicolumn{2}{|c|}{$(75.3 \%)$} & & & \\
\hline & $\mathrm{T}_{1}$ & $\begin{array}{r}260->262 \\
261<-262 \\
261->263 \\
(81 .\end{array}$ & $\begin{array}{r}0.26988 \\
0.13620 \\
0.63873 \\
0)\end{array}$ & 1.391 & & \\
\hline \multirow{3}{*}{700} & CT & $\begin{array}{r}260->263 \\
261->262 \\
(88 .\end{array}$ & $\begin{array}{r}-0.22128 \\
0.66360 \\
0)\end{array}$ & 2.741 & 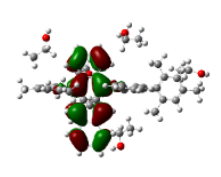 & 等 \\
\hline & $\begin{array}{c}\mathrm{S}_{1} \\
(\mathrm{LE})\end{array}$ & $\begin{array}{r}260->262 \\
(95 .\end{array}$ & 0.69033 & 2.921 & 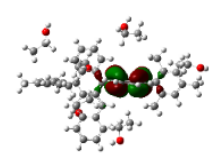 & 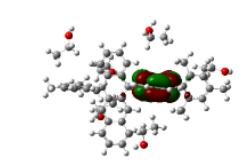 \\
\hline & $\mathrm{T}_{1}$ & $\begin{array}{r}261<-262 \\
261->263 \\
\end{array}$ & $\begin{array}{r}0.15685 \\
0.69380 \\
)\end{array}$ & 1.436 & 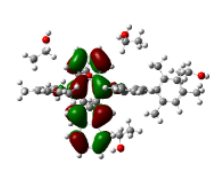 & 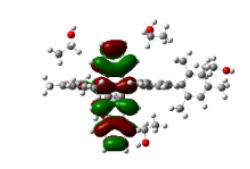 \\
\hline
\end{tabular}


Table S31. The Examples of Electron Transitions of Excited States and Their Corresponding Frontier Molecular Orbitals for DT-Mes in Ethanol with Virtual CT State $\left(\Delta \mathrm{E}_{\mathrm{SF}}<0, \Delta \mathrm{E}_{\mathrm{CT}-\mathrm{S} 1}>0\right.$ \& singlet strongly mixed with the nearby CT states)

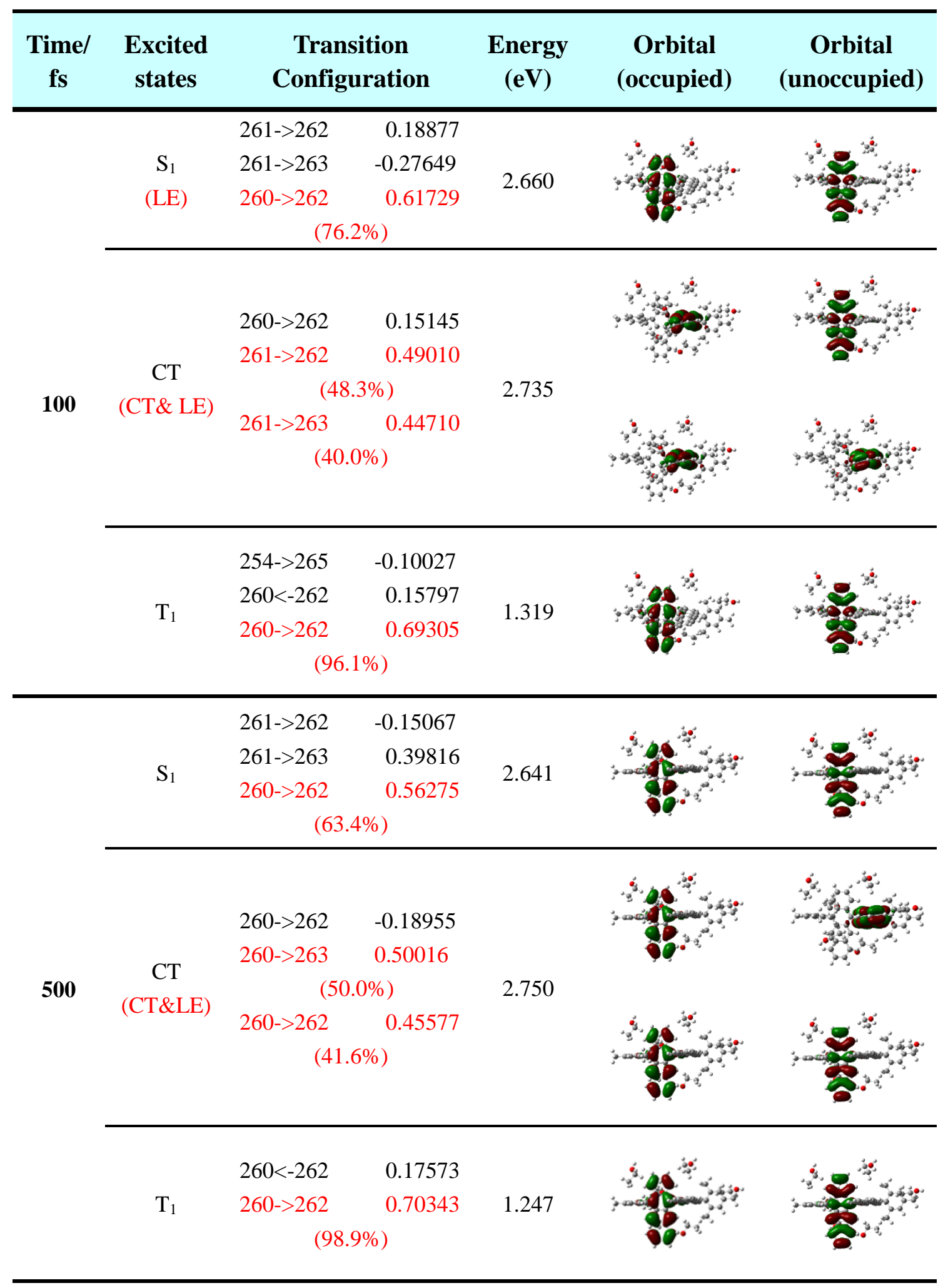


Table S32. Electron Transitions of Excited States and Their Corresponding Frontier Molecular Orbitals for DT-Mes in Ethanol No SF $\left(\Delta \mathrm{E}_{\mathrm{SF}}<0, \Delta \mathrm{E}_{\mathrm{CT}-\mathrm{S} 1}>0\right.$ but singlet not mixed with the CT states)

\begin{tabular}{|c|c|c|c|c|c|c|}
\hline $\begin{array}{l}\text { Time/ } \\
\text { fs }\end{array}$ & $\begin{array}{c}\text { Excited } \\
\text { states }\end{array}$ & $\begin{array}{r}\text { Transi } \\
\text { Configu }\end{array}$ & $\begin{array}{l}\text { tion } \\
\text { ration }\end{array}$ & $\begin{array}{c}\text { Energy } \\
(\mathrm{eV})\end{array}$ & $\begin{array}{c}\text { Orbital } \\
\text { (occupied) }\end{array}$ & $\begin{array}{c}\text { Orbital } \\
\text { (unoccupied) }\end{array}$ \\
\hline \multirow{3}{*}{600} & $\begin{array}{c}\mathrm{S}_{1} \\
(\mathrm{LE})\end{array}$ & $\begin{aligned} & 260-> 262 \\
& 261-> 263 \\
&(80.0\end{aligned}$ & $\begin{array}{l}0.30355 \\
0.63273 \\
\%)\end{array}$ & 2.704 & 28 & 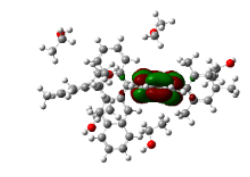 \\
\hline & $\mathrm{CT}$ & $\begin{aligned} & 260-> 263 \\
&(96.2\end{aligned}$ & $\begin{array}{l}0.69359 \\
\%)\end{array}$ & 2.976 & & 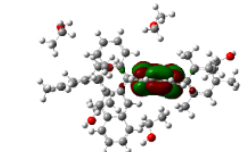 \\
\hline & $\mathrm{T}_{1}$ & $\begin{aligned} 259-> & 266 \\
261<- & 263 \\
261-> & 263 \\
& (95.7\end{aligned}$ & $\begin{array}{l}0.10451 \\
0.15668 \\
0.69163 \\
\text { ) }\end{array}$ & 1.325 & & \\
\hline \multirow{3}{*}{900} & $\begin{array}{c}\mathrm{S}_{1} \\
(\mathrm{LE})\end{array}$ & $\begin{array}{r}260->262 \\
261->262 \\
261->263 \\
(72.8\end{array}$ & $\begin{array}{r}0.31911 \\
0.16873 \\
0.60332 \\
\text { \%) }\end{array}$ & 2.779 & 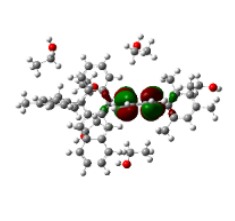 & \\
\hline & $\mathrm{CT}$ & $\begin{array}{r}260->262 \\
261->263 \\
261->262 \\
(77.2\end{array}$ & $\begin{array}{l}0.16968 \\
-0.26561 \\
0.62111 \\
\%\end{array}$ & 3.076 & 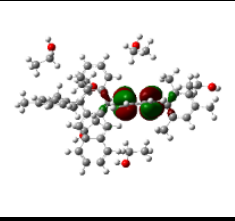 & \\
\hline & $\mathrm{T}_{1}$ & $\begin{array}{r}261->262 \\
261<-263 \\
261->263 \\
(86.1\end{array}$ & $\begin{array}{l}0.20200 \\
0.11974 \\
0.65608 \\
\text { \%) }\end{array}$ & 1.362 & 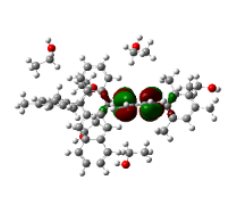 & 象 \\
\hline
\end{tabular}

